\title{
THE REPRESENTATION OF ABSTRACT MEASURE FUNCTIONS
}

BY

\author{
DOROTHY MAHARAM
}

\section{Introduction.}

1.1. Several authors have discussed abstract "measures" and "integrals," the values of which need not be numerical $\left({ }^{1}\right)$. Our object here is to show that, in a sense, such abstract measures and integrals are of ten equivalent (under fairly general hypotheses) to combinations of ordinary numerical ones. We shall here consider only the theory of measure, to which the theory of abstract-valued integration can in most cases be reduced. The main theorem (3.1 (f) and \$19) can be stated, roughly, as follows: a field of sets with an abstract measure on it is isomorphic to an ideal in the direct product of two such fields, on one of which the measure is an ordinary numerical one, while on the other factor the measure is trivial. (Precise definitions of the concepts involved will be given later.) As a consequence of this, the abstract measure can be replaced, to within isomorphism, by one whose values are (real-valued) numerical functions on a certain set, modulo a certain class of null functions.

We conclude the paper with a few deductions from this theorem, reserving however the application to abstract-valued integration for a subsequent paper.

1.2. Formulation of the problem. Instead of dealing directly with a field $F$ of sets, on which a suitable abstract-valued measure $\lambda$ is defined, it is more convenient to work with the Boolean algebra $E$ of the sets of $F$ modulo null sets-that is, with the algebra formed by the equivalence classes $\{x\}$ of sets belonging to $F$, where $y \in\{x\}$ means $\lambda\left(x+{ }_{2} y\right)=0\left({ }^{2}\right)$, the "measure" on $E$ being given by setting $\lambda(\{x\})=\lambda(x)$. (That this relation is an equivalence relation, that $\lambda(x)=\lambda(y)$ whenever $y \in\{x\}$, and that the equivalence classes $\{x\}$ do form a Boolean $\sigma$-algebra $\left(^{3}\right)$ are in effect included in our postulates on $\lambda$.) Thus we provisionally define an abstract measure algebra to be an ordered pair $(E, \lambda)$, where $E$ is a Boolean $\sigma$-algebra and $\lambda$ is an abstract measurefunction on $E$, satisfying "reasonable" postulates (as yet unspecified). Two abstract measure algebras $\left(E_{1}, \lambda_{1}\right)$ and $\left(E_{2}, \lambda_{2}\right)$ are defined to be isomorphic if there exists an isomorphism of the algebras $E_{1}$ and $E_{2}$, say $\phi\left(x_{1}\right)=x_{2}$, such

Presented to the Society, November 27, 1943; received by the editors January 14, 1948.

(1) We shall not be directly concerned here with abstract-valued integrals or with the closely related real-valued integrals of abstract-valued functions. For abstract-valued measures, see for example $[4],[5],[10]$, and $[14, \S 8]$ in the bibliography at the end of the paper.

(2) See 1.3 for the notations.

(3) See, for example [2, pp. 29, 88]. 
that $\lambda_{2}\left(\phi\left(x_{1}\right)\right)=\lambda_{2}\left(\phi\left(y_{1}\right)\right)$ if and only if $\lambda_{1}\left(x_{1}\right)=\lambda_{1}\left(y_{1}\right)$. If we further have $\lambda_{2}\left(\phi\left(x_{1}\right)\right)=\lambda_{1}\left(x_{1}\right)$ for a suitable isomorphism $\phi$, then $\left(E_{1}, \lambda_{1}\right)$ and $\left(E_{2}, \lambda_{2}\right)$ are said to be isometric.

We shall here be interested in abstract measure algebras only to within isomorphism; and this permits a further reduction of the problem. In $(E, \lambda)$ we define the "induced" equivalence relation, $\sim$, by writing $x \sim y$ to mean $\lambda(x)=\lambda(y)$. The properties of $(E, \lambda)$ to within isomorphism are evidently determined completely by this equivalence relation. Accordingly we define an $a b s t r a c t$ measure algebra to be either an ordered pair $(E, \lambda)$ as before, or an ordered pair $(E, \sim)$, where $E$ is a Boolean $\sigma$-algebra and $\sim$ is an equivalence relation on $E$ satisfying the postulates to be given below. If $\sim$ is the equivalence relation induced by $\lambda$, we say that $(E, \lambda)$ and $(E, \sim)$ are naturally isomorphic. The definition of isomorphism between two abstract measure algebras is now clear; for example, $\left(E_{1}, \sim\right)$ and $\left(E_{2}, \approx\right)$ will be isomorphic if and only if there exists a mapping $\phi$ of $E_{1}$ on $E_{2}$ such that (i) $\phi$ is an algebraic isomorphism and (ii) $\phi\left(x_{1}\right) \approx \phi\left(y_{1}\right)$ if and only if $x_{1} \sim y_{1}$.

1.3. Notations. As far as practicable, we adhere to the following notations. $E$ denotes a Boolean $\sigma$-algebra $\left({ }^{3}\right)$ having $o$ as zero element and $e$ as unit element. Small letters $a, b, x, y$, and so on, usually denote elements of $E$, except that $m, n, i, j$ usually denote positive integers; capital letters $X, Y$, and so on, usually denote subsets of $E$ (or of some other set). Phrases like " $x \in E, X \subset E$ " are generally omitted when the meaning is clear. The partial ordering relation in $E$ is written $\leqq$, as usual; thus $x<y$ means $x \leqq y$ and $x \neq y$. (But $X \subset Y$ does not exclude the equality of $X$ and $Y$.) The supremum of two elements of $E$ is written $x \vee y$, and of an infinite collection is written $\vee x_{i}$; infima are written $x y, \wedge x_{i}$. We use $a X$ to denote the set of all elements of the form $a x$, where $x \in X$.

If $x y=o$, we say that $x$ and $y$ are disjoint. A subset of $E$ is said to be disjoint if every two of its elements are disjoint.

The complement of $x$ is denoted by $-x$, and $x-y$ is used as an abbreviation for $x(-y)$. The symmetric difference of $x$ and $y$ is the element $(x-y) \vee(y-x)$; we shall usually denote this by $x+{ }_{2} y$ ("addition mod 2 ").

$E$ will generally be assumed to satisfy the countable chain condition, namely, there does not exist an uncountable disjoint subset of $E$. As is well known, this will imply that $E$ is complete (that is, that for every $X \subset E$ both $\vee\{x \mid x \in X\}$ and $\Lambda\{x \mid x \in X\}$ exist).

The words "algebra," "sub-algebra," used unqualified always refer to Boolean $\sigma$-algebras. A Boolean algebra in which only finite operations are to be considered will be called a "finitely additive algebra."

For any $a \in E$, the principal ideal $E(a)$ of $a$ in $E$ is the set of all $x \in E$ satisfying $x \leqq a . E(a)$ is evidently itself an algebra with $\leqq, \vee, \Lambda$, having the same meaning as in $E$, but having $a$ as unit element instead of $e$. A sub-algebra of $E(a)$ will be called a sub-algebra of $E$ relative to a. Similarly, if $F$ is a 
subalgebra of $E$, and $b \in F, F(b)$ denotes the principal ideal of $b$ in $F$; it is clear that $F(b)=b F$, but the notation $F(b)$ is used to emphasize that $b \in F$.

Given $X \subset E(a)$, the sub-algebra relative to $a$ which it generates is called the Borel field of $X$ relative to $a$, and is written $\mathscr{B}_{a}(X)$; it is thus the smallest set $B \subset E(a)$ which satisfies (i) $B \supset X$, (ii) if $x \in B$, then $a-x \in B$, (iii) if $x_{n} \in B(n=1,2, \cdots)$ then $\bigvee x_{n} \in B$. If in particular $a=e$, we omit the phrase "relative to $a$," and write the Borel field of $X$ as $\mathcal{B}(X)$.

The usual notations of set theory are employed for subsets of $E$ (or of other sets), the union of $X$ and $Y$ being written $X \cup Y$, and their intersection $X \cap Y$, and so on. The empty set is denoted by 0 . The cardinal number of a set $X$ is written $|X|$.

Greek letters are generally used as follows: $\alpha, \beta$, and so on, are ordinal numbers; $\lambda$ is an abstract measure, and $\mu$ is an ordinary numerical one; $\theta$, $\phi, \psi$, are mappings; $\rho, \sigma, \tau$, are non-negative real numbers not exceeding $1, \rho$ usually being rational; and $\epsilon$ is a positive number less than 1 .

Sequences (indicated by the notation $\left\{x_{n}\right\}$ ) can usually be either finite or (countably) infinite, except where the distinction is emphasized (for example, by writing $n=1,2, \cdots$, to $\infty)$.

Where the abstract measure or equivalence relation intended is clear, we of ten write $(E, \lambda)$ or $(E, \sim)$ simply as $E$.

\section{Postulates.}

2.1. Since the equivalence relation $\sim$, rather than the abstract measure $\lambda$, will be used in what follows, it is simpler to state our postulates in terms of $\sim$. (It would be easy but tedious to deduce them from "reasonable" postulates on $\lambda\left(^{4}\right)$; and conversely the main theorem of this paper, stated in 3.1(f), will show that every equivalence relation satisfying our postulates is in fact induced by such a "reasonable" $\lambda$.) The postulates for the abstract measure algebra $(E, \sim)$ are:

(0) $E$ is a Boolean $\sigma$-algebra satisfying the countable chain condition, and $\sim$ is an equivalence relation on $E$.

(I) If $x=\mathrm{V} x_{n}, y=\mathrm{V} y_{n}$, where $x_{m} x_{n}=o=y_{m} y_{n}(m \neq n ; m, n=1,2, \cdots)$, and if, for each $n, x_{n} \sim y_{n}$, then $x \sim y$.

(II) If $x \sim x^{\prime}$, and if $y \leqq x$ is given, there exists $y^{\prime} \leqq x^{\prime}$ such that $y \sim y^{\prime}$.

Before stating the last postulate, we need the following definition. An element $x$ is bounded if, whenever $y \leqq x$ and $y \sim x$, we have $y=x$.

(III) If $x \sim y$ there exist bounded elements $x_{n}, y_{n}(n=1,2, \cdots)$ such that $x=\mathrm{V} x_{n}, y=\mathrm{V} y_{n}, x_{m} x_{n}=o=y_{m} y_{n}(m \neq n)$, and $x_{n} \sim y_{n}$.

(4) The set of values taken by $\lambda$ would be, for example, a lattice-ordered Abelian semigroup. Postulate I, for example, would be replaced by the requirement that $\lambda$ be countably additive. One could deduce from [6, Theorem 10] that there is no loss in assuming the values of $\lambda$ to be equivalence classes of numerical functions; but we shall derive independently a more precise result than this. For the device of replacing $\lambda$ by $\sim$, cf. $[10]$ and (though with different objectives) $[4]$ and $[12, \mathrm{Ch} . \mathrm{VI}]$. 
2.2. Numerical case. To illustrate the meaning of these postulates, consider a "numerical measure algebra" $(E, \mu)$, in which $\sim$ is induced by an ordinary countably additive numerical function $\mu$, taking non-negative real values and possibly the value $\infty$. Suppose that there exists $x \neq 0$ for which $\mu(x)<\infty$. If the postulates 0 -III are satisfied it readily follows that the bounded elements are precisely those of finite measure, and thence that $\mu$ is $\sigma$-finite (that is, $e=\mathrm{V} a_{n}$ where $\left.\mu\left(a_{n}\right)<\infty\right)$. Further, $\mu$ must be "reduced" (that is, vanishes only for $o$ ). Conversely, if $\mu$ is a numerical countably additive measure on $E$, reduced and $\sigma$-finite, and if II holds, then elements of finite measure are certainly bounded, and postulates 0 , I and III readily follow. Postulate II, though not automatically satisfied, can easily be seen to hold if either (i) $E$ is non-atomic $\left(^{5}\right.$ ), or (ii) $E$ is generated by a number of atoms all of equal measure $\left.{ }^{6}\right)$. One would thus not expect II to be unduly restrictive in practice $\left({ }^{7}\right)$. There are, of course, important numerical measures which satisfy neither 0 nor III-for example, Hausdorff $m$-dimensional measure in Euclidean $n$-space $(n>m)\left({ }^{8}\right)$. In what follows, the phrase "numerical measure algebra" will mean (except where the contrary is explicitly stated) one in which the postulates are satisfied, and in which the measure takes at least one finite nonzero value.

We note that, for numerical measure algebras, isomorphism implies isometry, to within a nonzero constant numerical factor.

REMARK. It might seem preferable, in the interests of simplicity, to replace postulate III by the stronger requirement that all elements be bounded -corresponding in the numerical case to the restriction that $\mu(e)<\infty$. But this would be undesirable, apart from the loss of generality, since it turns out that even then it would be necessary to introduce numerical measure algebras in which some elements have infinite measure and are therefore unbounded.

2.3. Elementary properties. The following properties of an abstract measure algebra are easy deductions from the postulates.

(1) $e=\mathrm{V} a_{n}$, where the elements $a_{n}$ are bounded and disjoint.

(2) If $x$ is bounded and $y \leqq x$, then $y$ is bounded.

(3) $o$ is bounded; conversely, if $x \sim o$ and $x$ is bounded, then $x=o$.

(4) If $x \sim o$, then $x=o$. (From III, (3) and I.)

(5) An atom is an element $x \in E$ such that (i) $x \neq o$, (ii) if $o<y \leqq x$, then $y=x$.

${ }^{(6)}$ Conversely, it is easy to see that a numerical measure algebra which satisfies the postulates is of one of the following four types: (i) non-atomic; (ii) generated by $\aleph_{0}$ atoms of equal measure; (iii) the direct sum of a non-atomic algebra of finite total measure and of an algebra generated by a finite number of atoms; (iv) entirely generated by atoms and of finite total measure-the possibilities in this case can be further analyzed.

(7) Postulate II can be omitted if the values of the abstract measure $\lambda$ are assumed to lie in a vector $\sigma$-lattice, for it can then be shown that the abstract measure algebra can be imbedded in one which satisfies all the postulates.

(8) Examples of abstract measures not satisfying the postulates are: Choquet's $[4 ; 5]$, and Löwner's measure in Hilbert space (Ann. of Math. vol. 40 (1939) pp. 816-833). 
(5) Two given elements have maximal equivalent sub-elements. More precisely; given $a, b \in E$, there exist $x \leqq a, y \leqq b$ such that (i) $x \sim y$, (ii) if $h \leqq a-x$, $k \leqq b-y$, and $h \sim k$, then $h=k=0$.

(The elements $x$ and $y$ are constructed by a transfinite induction argument -or equivalently by using Zorn's lemma - the transfinite induction necessarily terminating countably because of the countable chain condition. This type of argument is often called the "method of exhaustion.")

\section{Fundamental examples.}

3.1. We shall here define some particular abstract measure algebras which will play a fundamental part in the sequel.

(a) A numerical measure algebra $(J, \mu)$ (cf. 2.2). In particular, important measure algebras satisfying the postulates are: the measure algebra $I^{1}$ $\left(=\left(I^{1}, \mu\right)\right)$ of Lebesgue measurable sets, modulo null sets, in the unit interval $(0,1)$; and the direct product $I^{\mathfrak{m}}$ of $\mathfrak{m}$ copies of $I^{1}$ (cf. $[8$, p. 419]), $\mathfrak{m}$ being any cardinal number. If $\mathfrak{m} \leqq \aleph_{0}, I^{1}$ and $I^{\mathfrak{m}}$ are well known to be isometric. Other measure algebras which will arise in the sequel are: $K$, the algebra generated by $\boldsymbol{\aleph}_{0}$ atoms all of measure 1 , and $L$, consisting of just two elements of measures 0 and 1 respectively.

(b) A "trivial" abstract measure algebra, U. By definition this is one in which no two distinct elements are equivalent $\left({ }^{9}\right)$. Obviously any algebra $U$ satisfying the countable chain condition can be regarded as a trivial abstract measure algebra.

(c) If $(E, \sim)$ is any abstract measure algebra, so is the relative algebra (principal ideal) $E(a)$ (cf. 1.3), in which the equivalence relation is defined in the "natural" way; $x \sim y$ in $E(a)$ (where $x, y \leqq a$ ) if and only if $x \sim y$ in $E$. The same is true, more generally, of any relative sub-algebra, provided that it satisfies postulate II.

(d) If $\left(E_{1}, \sim\right)$ and $\left(E_{2}, \approx\right)$ are abstract measure algebras (satisfying our postulates, as always), then so is their "free direct sum." This is defined as follows. The algebra is the "direct sum" $E_{1} \oplus E_{2}$-that is, the set of ordered pairs $\left(x_{1}, x_{2}\right)$ with $x_{1} \in E_{1}, x_{2} \in E_{2}$, the ordering relation being given by: $\left(x_{1}, x_{2}\right) \leqq\left(y_{1}, y_{2}\right)$ if and only if we have both $x_{1} \leqq y_{1}$ and $x_{2} \leqq y_{2}$. The equivalence relation in the direct sum is then given by: $\left(x_{1}, x_{2}\right)$ and $\left(y_{1}, y_{2}\right)$ are equivalent if and only if we have both $x_{1} \sim y_{1}$ and $x_{2} \approx y_{2}$.

REMARK. This "free direct sum" is to be distinguished from the ordinary "direct sum" of two numerical measure algebras. There the algebra is the same $\left(E_{1} \oplus E_{2}\right)$, but the equivalence relation is different, being induced by the measure $\mu$, where $\mu\left(x_{1}, x_{2}\right)=\mu_{1}\left(x_{1}\right)+\mu_{2}\left(x_{2}\right), \mu_{1}$ and $\mu_{2}$ being the measures in $E_{1}$ and $E_{2}$.

(e) A more complicated example is the "direct product" $J \otimes U$ of a numerical measure algebra $J=(J, \mu)$ (of type (a) above) and a trivial algebra $U$ (of

(9) A numerical measure algebra can be trivial in this sense, though only if it is generated by atoms. Examples: $L$, and the algebra generated by a sequence of atoms of measures $3^{-n}$. 
type (b)). This may be characterized as follows. Suppose $(E, M)$ is an abstract measure algebra in which the values of the abstract measure $M$ are non-negative numerical functions on some set, modulo some class of null functions (for example, continuous functions on some space modulo functions which vanish except for sets of first category). Suppose $E$ contains two subalgebras $J, U$, such that:

(i) $E=\mathscr{B}(J \cup U)$.

(ii) If $x \in J, M(x)$ is a constant function, $=\mu(x)$ say.

(iii) If $x \in U, M(x)=\mu(e)$ times the characteristic function of some set (with the convention that $\infty \cdot 0=0$ ).

(It is further supposed, of course, that $M$ is countably additive in the obvious sense, and that postulates 0 -III are satisfied.) Then the sub-algebra $(J, M)$ is clearly isomorphic with a numerical measure algebra $(J, \mu)\left({ }^{10}\right)$; and it is not hard to show that the sub-algebra $(U, M)$ is trivial. It further follows that, for any $x \in J$ and $u \in U, M(x u)=\mu(x) M(u)$. We say that $(E, M)$ is the direct product of $(J, \mu)$ and $U$.

It will be proved in the next section that two given algebras $(J, \mu)$ and $U$ always have a direct product. This product is moreover unique to within isomorphism; we shall not prove this explicitly, but it follows by essentially the same arguments as in $\$ 17$.

(f) Finally, it is evident that a principal ideal (that is, relative algebra) in the free direct sum of two direct products of type (e) will be an abstract measure algebra. Our main theorem is that the converse is true; every abstract measure algebra is isomorphic to one of this type. (In fact, every abstract measure algebra is isomorphic to a principal ideal in a single direct product $J \otimes U$ of type (e) except that $J$ and $J \otimes U$ need not satisfy postulates II and III above.) Thus the four concepts $(E, \lambda),(E, \sim)$, abstract measure algebra of type (f), and $(E, \lambda)$ in which the values of $\lambda$ are numerical functions (modulo null functions), are all equivalent to within isomorphism.

We shall also be able to deduce some simpler characterizations in some important special cases $(\$ 20)$.

4. Construction of the direct product $J \otimes U$.

4.1. In this section we show that a given numerical measure algebra $J=(J, \mu)$ and a given trivial abstract measure algebra $U$ (satisfying our postulates) always have a direct product in the sense of 3.1(e). To save the trouble of proving explicitly that the direct product in the sense of $3.1(\mathrm{e})$ is unique (to within isomorphism), we shall use the unqualified term "direct product" in the rest of the paper to refer to the particular direct product to be constructed in this section. As the construction will depend on the notion of the "representation space" of an algebra, we begin by outlining the relevant properties.

4.2. Representation spaces. Let $E$ be any Boolean $\sigma$-algebra, with or with-

(10) Provided $\mu$ is not always 0 or $\infty$. 
out a measure. Then $\left({ }^{11}\right)$ there exists a compact (= bicompact) totally disconnected Hausdorff space $R$, the representation space of $E$, such that $E$ is isomorphic to the Boolean algebra formed by the "open-closed" (=simultaneously open and closed) subsets of $R$. Let $x^{*}$ denote the open-closed subset of $R$ which corresponds to $x \in E$ in this isomorphism. Then we have

(1) The sets $x^{*}$ form a basis for the open sets of $R$.

We note that for finite operations the "expected" relations hold; that is, $o^{*}=0, e^{*}=R,(-x)^{*}=R-x^{*}, x \leqq y \rightleftarrows x^{*} \subset y^{*},(x \vee y)^{*}=x^{*} \cup y^{*}$, and $(x y)^{*}$ $=x^{*} \cap y^{*}$. For infinite operations the correspondence is more complicated, but it is easily seen from the laws for finite operations that

(2) $\left(\bigvee x_{n}\right)^{*}=U x_{n}^{*} \cup z,\left(\bigwedge x_{n}\right)^{*}=\cap x_{n}^{*}-t$,

where $z$ and $t$ are nowhere dense closed subsets of $R$ (and are thus of the first category).

The Borel field $\mathcal{B}(R)$ of $R$ is defined (cf. 1.3) to be the smallest family $\mathscr{B}^{\prime}$ of subsets of $R$ such that (i) $\mathcal{B}^{\prime}$ contains all open-closed sets, (ii) if $X \in \mathcal{B}^{\prime}$, $R-X \in \mathcal{B}^{\prime}$, (iii) if $X_{n} \in \mathcal{B}^{\prime}(n=1,2, \cdots), \cup X_{n} \in \mathcal{B}^{\prime}$. We have:

(3) Any given $X \in B(R)$ can be written in one and only one way as $X=x^{*}$ $+{ }_{2} z$, where $x^{*}$ is open-closed and $z$ is of the first category $\left({ }^{12}\right)$.

For from (2) it is clear that the family $\mathcal{B}^{\prime}$ of all sets so expressible as $x^{*}+{ }_{2} z$ satisfies (i), (ii) and (iii); hence it contains $B(R)$. To see that the expression is unique, we note that if $x^{*}+{ }_{2} z=y^{*}+{ }_{2} t$ (where $x^{*}, y^{*}$ are open-closed and $z, t$ are of the first category) then $z+{ }_{2} t=x^{*}+{ }_{2} y^{*}$, an open set; and since a nonempty open set is not of the first category we must have $z+{ }_{2} t=0=x^{*}+{ }_{2} y^{*}$, that is, $z=t$ and $x^{*}=y^{*}$.

Now assume that $E$ satisfies the countable chain condition, and is thus complete. From (1) and (2) it now follows that

(4) If $G$ is an open subset of $R$, so is its closure $\bar{G}$. As a corollary,

(5) If $G_{1}, G_{2}$ are disjoint open subsets of $R$, so are $\bar{G}_{1}$ and $\bar{G}_{2}$.

We shall be interested in numerical functions $f$ on $R$ whose values are between 0 and $\infty$ inclusive; for brevity we refer to them simply as "functions." The continuity of functions is defined in the obvious way $\left.{ }^{13}\right)$, and a function $f$ is said to be continuous on $X \subset R$ if $f$, considered only for the points of $X$, is continuous relative to the subspace $X$.

(6) Lemma. Given a function $f$ on $R$ which is continuous on $R-H$, where $H$ is of the first category, there exists one and only one function $g$, continuous on $R$, such that $g(p)=f(p)$ for each $p \in R-H$.

Given $p \in H$, we assert $\lim \sup f(q)=\lim \inf f(q)$ as $q \rightarrow p$ through points

(11) See [15, Theorem 67].

$\left.{ }^{(12}\right)$ Thus $E$ is $\sigma$-isomorphic, in a natural way, to the algebra formed by $\mathcal{B}(R)$ modulo sets of first category-proving the known theorem (cf. [9]) that the notions "Boolean $\sigma$-algebra" and " $\sigma$-field of sets modulo a $\sigma$-ideal" are equivalent.

(13) If $f(p)=\infty$, continuity of $f$ at $p$ means that, given any $n$, there exists a neighborhood of $p$ throughout which $f>n$. 
of $R-H\left({ }^{14}\right)$. For if not, there exist (finite) real numbers $\rho, \sigma$ such that $\lim \sup f(q)>\rho>\sigma>\lim \inf f(q)$. Define $K_{1}=\{q \mid q \in R-H$ and $f(q)>\rho\}$, $K_{2}=[q \mid q \in R-H$ and $f(q)<\sigma\}$. From the continuity of $f, K_{1}$ and $K_{2}$ are open relative to $R-H$; hence $K_{i}=G_{i} \cap(R-H)$ where $G_{i}$ is open in $R(i=1,2)$. Since $K_{1} \cap K_{2}=0$, we have $G_{1} \cap G_{2} \subset H$; and since a non-empty open set is not of the first category in $R$, it follows that $G_{1} \cap G_{2}=0$. Hence (by (5)) $\bar{G}_{1} \cap \bar{G}_{2}=0$, contradicting $p \in \bar{K}_{1} \cap \bar{K}_{2} \subset \bar{G}_{1} \cap \bar{G}_{2}$.

Define $g$ by: $g(p)=f(p)$ for $p \in R-H$, and $g(p)=\lim f(q)$ for $p \in H$ (where $q \rightarrow p$ in $R-H$ ). It is easy to see that $g$ is continuous on $R$. Thus the required function exists; and its uniqueness is immediate.

(7) LEMmA. Let $\left\{f_{n}\right\}$ be a given sequence of continuous functions on $R$ such that, for each $p \in R, \vec{f}_{1}(p) \leqq f_{2}(p) \leqq \cdots$. Then there exists one and only one continuous function $f$ on $R$ such that $f(p)=\lim f_{n}(p)$ for each $p \in R-H, H$ being $a$ set of the first category.

Let $f^{\prime}(p)=\lim f_{n}(p)$; from the previous lemma it is enough to show that $f^{\prime}$ is continuous on $R-H$ where $H$ is of the first category. We can suppose that $f_{n}$ and $f^{\prime}$ are all uniformly bounded (on replacing $f_{n}$ by $f_{n} /\left(1+f_{n}\right)$ ).

For each positive integer $k$, let $\left\{x_{k t}^{*}\right\}(i=1,2, \cdots)$ be a maximal disjoint collection (necessarily countable) of nonempty open-closed subsets of $R$, each having the property that there exists an integer $n=n(k, i)$ such that $f^{\prime}(p) \leqq f_{n}(p)+1 / k$ whenever $p \in x_{k i}^{*}$. Write $G_{k}=\bigcup_{i} x_{k i}^{*}$; clearly $G_{k}$ is open. We assert $G_{k}=R$. For suppose not. Define $F_{n k}$ to be the set of all points $p \in R$ such that $f_{m}(p) \leqq f_{n}(p)+1 / k$ whenever $m \geqq n$; evidently $F_{n k}$ is closed, and $\bigcup_{n} F_{n k}=R$. Hence one of the sets $\left(R-G_{k}\right) \cap F_{n k}(n=1,2, \cdots)$ must be somewhere dense, and so (from (4)) must contain a nonempty open-closed set $y_{k}^{*}$. But $y_{k}^{*}$ is disjoint from every $x_{k i}^{*}(i=1,2, \cdots)$, and yet satisfies the requirement which was imposed on the sets $x_{k t}^{*}$; and this contradicts the maximality of the collection $\left\{x_{k i}^{*}\right\}$.

Since $\overline{G_{k}}=R$, the set $H=\bigcup_{k}\left(R-G_{k}\right)$ is of the first category. Suppose $p \notin H$; then, for every $k, p$ belongs to some $x_{k i}^{*}$. Writing $n$ for $n(k, i)$, we see from the continuity of $f_{n}$ that there is an open-closed set $y^{*}$ such that $p \in y^{*} \subset x_{k l}^{*}$ and, whenever $q \in y^{*}, \quad\left|f_{n}(q)-f_{n}(p)\right|<1 / k$. Thus $\left|f^{\prime}(q)-f^{\prime}(p)\right|<3 / k$ for all $q \in y^{*}$. That is, $f^{\prime}$ is continuous at every point of $R-H$, and is a fortiori continuous on $R-H$. Q.E.D.

REMARK. Arguments similar to the foregoing-which is evidently analogous to the proof of Egoroff's theorem-will occur again; we shall refer to them as "Egoroff arguments."

4.3. Now let $(J, \mu)$ be a numerical measure algebra, and let $U$ be a trivial abstract measure algebra. (It is assumed that neither $J$ nor $U$ consists of $o$ only.) Let $R$ and $S$ be the representation spaces of $J$ and $U$ respectively, and

(14) Convergence is in the sense of Moore and Smith; cf. [3]. 
let $T=R \times S$ (the space formed by the ordered pairs $(r, s), r \in R, s \in S$, with the obvious definition of the topology). Define the restricted Borel field $\mathcal{B}_{r}(T)$ to be the Borel field generated (in the algebra of subsets of $T$ ) by the "rectangles" $x^{*} \times u^{*}, x^{*}$ and $u^{*}$ being open-closed subsets of $R$ and $S$ respectively. For each restricted Borel set $H \in \mathcal{B}_{r}(T)$ and for each $s \in S$ define $K(H, s)$ to be the set of all points $r \in R$ such that $(r, s) \in H$. It is easily seen that $K(H, s) \in \mathcal{B}(R)$ (as follows, for example, by the same reasoning as in 8.5 below). Hence (from (3) above) each $K(H, s)$ can be uniquely expressed as $x^{*}+{ }_{2} z$, where $x^{*}$ is open-closed in $R$ and $z$ is of the first category in $R$. The set $x^{*}$ corresponds to a unique element $x \in J$, with measure $\mu(x)$. Thus we can define a function $M_{H}^{\prime}$ on $S$ by setting

(8) $M_{H}^{\prime}(s)=\mu(x)$, where $x^{*}+{ }_{2} z=K(H, s)$.

We next show that

(9) There exists a function $M_{H}$, differing from $M_{H}^{\prime}$ on a set of first category (at most) in $S$, and continuous on $S$. (Obviously $M_{H}$ will then be unique.)

Let $\left\{a_{n}\right\}$ be a disjoint sequence of elements of $J$ such that $\mu\left(a_{n}\right)<\infty$ and $V a_{n}=j$, the unit-element in $J$. (The existence of such a sequence follows from the fact that $J$ satisfies our postulates; cf. 2.3(1).) For each $H \in \mathcal{B}_{r}(T)$, define $H_{n}=H \cap\left(a_{n}^{*} \times S\right)$; thus the sets $H_{n}$ belong to $\mathcal{B}_{r}(T)$, are disjoint (for fixed $H$ ), and their union is $H$-a set of first category (from (2)). Hence $M_{H}^{\prime}=\sum_{n} M_{H_{n}}^{\prime}$. By (7) it will thus suffice to prove that, for each $n, M_{H_{n}}^{\prime}$ differs from a continuous function only on a set of first category in $S$. Consider the family $\mathcal{B}^{\prime}$ of those sets $H \in \mathcal{B}_{r}(T)$ for which this is true. Clearly $\mathcal{B}^{\prime}$ contains the "rectangles" $x^{*} \times u^{*}$; also if $H^{\prime} \supset H^{\prime \prime}$ and $H^{\prime}, H^{\prime \prime} \in \mathcal{B}^{\prime}$ then $H^{\prime}-H^{\prime \prime}$ $\in B^{\prime}$ (for $M_{\left(H^{\prime}-H^{\prime \prime}\right)_{n}}^{\prime}=M_{H_{n}^{\prime}}^{\prime \prime}-M_{H_{n}^{\prime \prime}}^{\prime \prime}$ since these functions are finite); and finally if $\left\{H^{m}\right\}$ is a disjoint sequence of sets in $\mathbb{B}^{\prime}$ it readily follows from (7) again that $U H^{m} \in \mathcal{B}^{\prime}$. Hence $\mathscr{B}^{\prime} \supset \mathcal{B}_{r}(T)$, and (9) is established.

We now define $J \otimes U$ to consist of the algebra $B_{r}(T)$ modulo "null sets"those for which $M_{H} \equiv 0$. Formally, we write $H \equiv H^{\prime}$ to mean that $H$, $H^{\prime} \in \mathcal{B}_{r}(T)$ and $M_{H+{ }_{2} H^{\prime}}=0$ for every $s \in S$-that is, that, except for a first category set of $s$ 's, each set $K(H, s)+{ }_{2} K\left(H^{\prime}, s\right)$ is of the first category in $R$ and write $\{H\}=$ set of all sets $H^{\prime}$ such that $H^{\prime} \equiv H$. Algebraic operations on the equivalence classes $\{H\}\left(H \in \mathcal{B}_{r}(T)\right)$ are defined in the obvious way; it is easily seen that they form a Boolean $\sigma$-algebra. On this we define an abstract measure by setting $M\{H\}=M_{H}$ (which also $=M_{H^{\prime}}$ if $H^{\prime} \equiv H$ ); thus the values of the "measure" are continuous functions on $S$. This measure is evidently countably additive, provided that we define $\sum_{n=1}^{\infty} M\left\{H_{n}\right\}$ to mean not the ordinary sum of these functions, but the continuous function which differs from this sum on a set of the first category (at most).

An alternative procedure would be to redefine the "measure" of $\{H\}$ to be the set of all functions which differ from $M\{H\}$ on an at most first category subset of $S$; addition would have then have its natural meaning. This is in fact the procedure which was used in the formulation in $3.1(\mathrm{e})$; however, the 
choice of $M\{H\}$ to be a single continuous function is more convenient in proving that $J \otimes U$ has the desired properties.

4.4. It is easy to see that $J \otimes U$ (assuming it for the moment to satisfy our postulates) is in fact (to within isomorphism) a "direct product" of $(J, \mu)$ and $U$ in the sense of 3.1 (e). For $J \otimes U$ contains a sub-algebra $J^{\prime}$ consisting of all elements of the form $\left\{x^{*} \times S\right\}$ ( $x^{*}$ open-closed in $R$ ), and a sub-algebra $U^{\prime}$ consisting of the elements $\left\{R \times u^{*}\right\}\left(u^{*}\right.$ open-closed in $\left.S\right)$. The function $M\left\{x^{*} \times S\right\}$ has evidently the constant value $\mu(x)$, and similarly $M\left\{R \times u^{*}\right\}$ has the value $\mu(j)$ for $s \in u^{*}$ (where $j$ denotes the unit element of $J$ ) and the value 0 for $s \in S-u^{*}$. Thus $\left(J^{\prime}, M\right)$ is isomorphic to the given measure algebra $(J, \mu)$, and $\left(U^{\prime}, M\right)$ is isomorphic to the given trivial algebra $U$. All that remains to be verified is that $J \otimes U=\mathscr{B}\left(J^{\prime} \cup U^{\prime}\right)$. To see this, consider the family $\mathcal{B}^{\prime}$ of all sets $H \in \mathcal{B}_{r}(T)$ for which the elements $\{H\}$ belong to $\mathbb{B}\left(J^{\prime} \cup U^{\prime}\right)$. Clearly $\mathcal{B}^{\prime}$ contains the "rectangles" $x^{*} \times u^{*}$, and contains $T-H$ and $U H_{n}$ if it contains $H$ and $H_{n}(n=1,2, \cdots)$. Hence $\mathcal{B}^{\prime} \supset \mathcal{B}_{r}(T)$, and so $\mathcal{B}^{\prime}=\mathscr{B}_{r}(T)$, from which it follows that $\mathbb{B}\left(J^{\prime} \cup U^{\prime}\right)=J \otimes U$.

4.5. We must now verify that $J \otimes U$ satisfies our postulates $(2.1)$, “ " being, of course, the equivalence relation induced by the abstract measure $M$.

(0) All that has to be proved here is that $J \otimes U$ satisfies the countable chain condition. Suppose there exists an uncountable disjoint collection of elements $\left\{H_{\alpha}\right\}$, all different from the zero element. As in the proof of (9) (4.2), we choose a disjoint sequence $\left\{a_{n}\right\}$ in $J$ with $\mu\left(a_{n}\right)<\infty$ and $V a_{n}=j$. For some $n$, there will be uncountably many nonzero elements $\left\{H_{\alpha} \cap\left(a_{n}^{*} \times S\right)\right\}$, and from these we obtain a transfinite sequence of strictly increasing elements $\left\{L_{\beta}\right\}$, $\beta<\omega_{1},\left\{L_{1}\right\}<\left\{L_{2}\right\}<\cdots<\left\{\left(a_{n}^{*} \times S\right)\right\}$. For each $\beta<\omega_{1}$, we have $M\left\{L_{\beta}\right\}$ $<M\left(L_{\beta+1}\right\}$ for at least one $s \in S$; and since these functions are continuous there exists a nonempty open-closed set $u_{\beta}^{*} C S$ such that $M\left\{L_{\beta}\right\}<\rho_{\beta}<\sigma_{\beta}$ $<M\left\{L_{\beta+1}\right\}$, where $\rho_{\beta}, \sigma_{\beta}$ are fixed positive numbers, for all $s \in u_{\beta}^{*}$. For some positive integer $m$ there will be uncountably many values (say) $\gamma$ of $\beta$ for which $\sigma_{\beta}-\rho_{\beta}>\mu\left(a_{n}\right) / m$. Thus no $s \in U u_{\gamma}^{*}$ can belong to more than $m$ sets $u_{\gamma}^{*}$. Let $v^{*}(s)$ denote the intersection of all sets $u_{\gamma}^{*}$ containing $s$; thus $v^{*}(s)$ is openclosed in $S$, and distinct sets $v^{*}(s)$ are disjoint. Since $U$ satisfies the countable chain condition, the distinct sets $v^{*}(s)$ must be countable in number; and since each $v^{*}(s)$ is contained in $u_{\gamma}^{*}$ for at most $m$ values of $\gamma$, the $\gamma$ 's are countablea contradiction.

(I) This is trivial here, since $M$ is countably additive.

(II) Given $\{H\},\left\{H^{\prime}\right\} \in J \otimes U$ such that $M\{H\}=M\left\{H^{\prime}\right\}$, and given $L^{\prime} \in \mathcal{B}_{r}(T)$ such that $\left\{L^{\prime}\right\} \leqq\left\{H^{\prime}\right\}$, we must show that there exists $L \in \mathcal{B}_{r}(T)$ such that $L \subset H$ and $M\{L\}=M\left\{L^{\prime}\right\}$. The proof is built up by first considering two special cases.

(i) Suppose $J$ has no atoms. We need the following lemma:

(10) Given $H \in \mathcal{B}_{r}(T)$, and given any continuous function $\phi$ on $S$, not identically zero, such that $0 \leqq \phi \leqq M\{H\}$ (for all $s \in S$ ), there exists $L_{1} \in \mathcal{B}_{r}(T)$ 
such that $L_{1} \subset H,\left\{L_{1}\right\} \neq 0$, and $M\left\{L_{1}\right\} \leqq \phi$.

For, from the continuity of $\phi$, there exists a nonempty open-closed set $u^{*} \subset S$ and an $\epsilon>0$ such that $\phi(s) \geqq \epsilon$ whenever $s \in u^{*}$. Let $\left\{c_{n}\right\}$ be a maximal disjoint collection of nonzero elements of $J$ each of measure less than $\epsilon$; since $J$ is non-atomic, it is easily seen that $\mathrm{V} c_{n}=j$. Write $L^{n}=H \cap\left(c_{n}^{*} \times u^{*}\right)$. Since $\sum_{n} M\left\{L^{n}\right\}=M\{H\} \geqq \phi$ (except on a first category set), there exists an $n$ for which $M\left\{L^{n}\right\}$ is not identically 0 , and we have only to take $L_{1}=L^{n}$.

The usual "exhaustion" argument now shows that, under the hypotheses of the lemma, there exists $L \in \mathcal{B}_{r}(T)$ such that $L \subset H$ and $M\{L\}=\phi$. (Take $L$ to be a maximal $L_{1}$, modulo null sets; if the function $\phi^{\prime}=\phi-M\{L\}$ were not identically 0 , with the convention $\infty-\infty=0$, the lemma applied to the set $H-L$ and function $\phi^{\prime}$ would enable $L$ to be enlarged.) In particular, on taking $\phi=M\left\{L^{\prime}\right\}$, we see that II is established in this case.

(ii) Suppose $J$ is generated by a number of atoms, all of equal measure. The proof proceeds as in case (i), the only difference being that we now restrict the continuous function $\phi$ to have only integral multiples of the measure of each atom (or $\infty$ ) as values.

(iii) In the general case, there are at most $\boldsymbol{\aleph}_{0}$ atoms in $J$; let the distinct values of their measures be enumerated as $\mu_{1}, \mu_{2}$, and so on. Write $b_{n}=$ supremum of all atoms in $J$ of measure $\mu_{n}$, and $b_{0}=j-\bigvee b_{n}$. For each $X \in \mathcal{B}_{r}(T)$ write $X^{n}=X \cap\left(b_{n}^{*} \times S\right)$. It is not hard to see, from the fact that $J$ itself satisfies II, that if $M\{H\}=M\left\{H^{\prime}\right\}$ then $M\left\{H^{n}\right\}=M\left\{H^{\prime n}\right\}$ for each $n$ $(n=0,1, \cdots)\left({ }^{15}\right)$. Now, given $\left\{L^{\prime}\right\} \leqq\left\{H^{\prime}\right\}$, we apply the argument in case (i) to obtain $L^{0} \subset H^{0}$ such that $M\left\{L^{0}\right\}=M\left\{L^{\prime 0}\right\}$, and similarly apply the argument in case (ii) to obtain $L^{n} \subset H^{n}$ such that $M\left\{L^{n}\right\}=M\left\{L^{\prime n}\right\}(n \geqq 1)$. Write $L=U L^{n}(n \geqq 0)$; evidently II follows.

(III) Given $H, H^{\prime} \in \mathcal{B}_{r}(T)$ such that $M\{H\}=M\left\{H^{\prime}\right\}$, we decompose them into equivalent bounded elements as follows. As before, let $\left\{a_{n}\right\}$ be a disjoint sequence of elements of $J$ such that $\mu\left(a_{n}\right)<\infty$ and $\mathrm{V} a_{n}=j$. Write $X_{n}=H \cap\left(a_{n}^{*} \times S\right), Y_{n}=H^{\prime} \cap\left(a_{n}^{*} \times S\right)$. Define $H_{1}=X_{1}$. From postulate II, there exists $H_{1}^{\prime} \subset H^{\prime}$ such that $M\left\{H_{1}^{\prime}\right\}=M\left\{H_{1}\right\} \leqq \mu\left(a_{1}\right)$; hence $M\left\{H-H_{1}\right\}$ $=M\left\{H^{\prime}-H_{1}^{\prime}\right\}$. Define $H_{2}^{\prime}=Y_{1} \cap\left(T-H_{1}^{\prime}\right)$; from II again there exists $H_{2} \subset H-H_{1}$ such that $M\left\{H_{2}\right\}=M\left\{H_{2}^{\prime}\right\}<\infty$, so that $M\left\{H-\left(H_{1} \cup H_{2}\right)\right\}$ $=M\left\{H^{\prime}-\left(H_{1}^{\prime} \cup H_{2}^{\prime}\right)\right\}$. Define $H_{3}=X_{2} \cap\left(T-\left(H_{1} \cup H_{2}\right)\right)$; and so on. We thus obtain disjoint sequences $\left\{H_{n}\right\},\left\{H_{n}^{\prime}\right\}$ such that $H=\bigcup H_{n}, H^{\prime}=\bigcup H_{n}^{\prime}$; and $M\left\{H_{n}\right\}=M\left\{H_{n}^{\prime}\right\}<\infty$, so that the elements $\left\{H_{n}\right\},\left\{H_{n}^{\prime}\right\}$ are bounded, as required.

REMARK. The construction of $J \otimes U$ will apply even if $J=(J, \mu)$ is an arbitrary numerical measure algebra in which $\mu$ is $\sigma$-finite. Naturally, if $J$ does not satisfy the postulates, $J \otimes U$ need not either.

(15) Let $K(H, s)=x^{*}+{ }_{2} z, K\left(H^{\prime}, s\right)=x^{*}+{ }_{2} z^{\prime}$; then (ignoring a first category set of $s^{\prime}$ s) $\mu(x)=\mu\left(x^{\prime}\right)$. If $x \geqq$ an atom of measure $\mu_{1}, x^{\prime} \geqq$ an element of $J$ of measure $\mu_{1}$, which must also be an atom. Iteration of this argument gives $\mu\left(b_{n} x\right)=\mu\left(b_{n} x^{\prime}\right)(n=0,1, \cdots)$, and the result follows. 
4.6. We conclude this section by noting, for future use, that the elements of $J \otimes U$ are (roughly speaking) approximable by countable sums of rectangles. More precisely:

(11) Lemma. Suppose $\mu(j)<\infty$. Then, given $H \in \mathcal{B}_{r}(T)$ and $\epsilon>0$, there exist sequences $x_{n}^{*}, u_{n}^{*}$ of open-closed subsets of $R$ and $S$ respectively, such that (i) the sets $u_{n}^{*}$ are disjoint and cover $S$ except for a set of first category, and (ii) we have

$$
M\left\{H+{ }_{2} \cup\left(x_{n}^{*} \times u_{n}^{*}\right)\right\}<\epsilon \quad(\text { for all } s \in S)\left({ }^{16}\right) .
$$

Consider the family $\mathcal{B}^{\prime}$ of sets $H \in \mathcal{B}_{r}(T)$ for which this is true (for every $\boldsymbol{\epsilon})$. Clearly $B^{\prime}$ contains the "rectangles" $x^{*} \times u^{*}$. It is also easy to see that if $H, H^{\prime} \in \mathcal{B}^{\prime}$ then so do $T-H$ and $H \cup H^{\prime}$; thus $\mathbb{B}^{\prime}$ is a finitely additive field, and to prove $\mathscr{B}^{\prime}=\mathscr{B}_{r}(T)$ it will suffice to prove that if $H_{n} \in \mathcal{B}^{\prime}$ and $H_{1} \subset H_{2} \subset \cdots$, then $\cup H_{n} \in \mathcal{B}^{\prime}$. This follows by an "Egoroff argument" (cf. the proof of $4.2(7))$.

5. Invariant elements.

5.1. Definition. Returning now to an abstract measure algebra $(E, \sim)$ satisfying the postulates of 2.1 , we define an element $u$ to be invariant if, whenever $x \sim u$, we have $x \leqq u$. (Thus if $u$ is invariant and bounded, we have further $x=u$.) Clearly no two distinct invariant elements can be equivalent. (The term "invariant" will be justified in 20.4(1).)

The invariant elements will play a fundamental part in the analysis of the structure of $(E, \sim)$. In fact, if $E$ were a non-atomic numerical measure algebra, the only invariant elements would evidently be $o$ and $e$; and this suggests, as is in fact the case, that the "trivial" factors in the desired "product" representation of $E$ (cf. 3.1(f)) in the general case, will be formed from invariant elements.

The set of all invariant elements of $E$ will be written $U$.

5.2. If $u$ is invariant and $x \sim x^{\prime} \leqq u$, then $x \leqq u$.

Write $y=x-u$; by II (2.1) there exists $y^{\prime} \leqq x^{\prime}$ such that $y \sim y^{\prime}$. Now let $z=\left(u-y^{\prime}\right) \bigvee y$. From $\mathrm{I}(2.1) z \sim\left(u-y^{\prime}\right) \bigvee y^{\prime}=u$; hence, from the definition of invariance, $z \leqq u$. A fortiori $y \leqq u$, and so $y=o$ and $x \leqq u$.

5.3. If $u$ is not invariant, there exist nonzero $x, y$ such that $x \leqq u, y \leqq-u$, and $x \sim y$.

For there exists $x_{1} \sim u$ such that $x_{1}-u \neq 0$. Define $y=x_{1}-u$. By $\operatorname{II}(2.1)$ there exists $x \leqq u$ such that $x \sim y$ (for $y \leqq x_{1} \sim u$ ).

5.4. The invariant elements form a complete Boolean sub-algebra of $E$.

It is trivial that $o$ and $e$ are invariant (2.3(4)). Thus we have only to prove (a) if $u$ is invariant so is $-u$, and (in view of the countable chain condition) (b) if $u_{n}$ is invariant $(n=1,2, \cdots)$ then so is $V u_{n}$. Now (a) is an immediate consequence of 5.3 and 5.2. To prove (b), let $u=\bigvee u_{n}$, and suppose

(16) This lemma can easily be modified so as to apply even if $\mu(j)=\infty$. Both lemma and proof can also be reformulated in terms of the properties given in 3.1 (e). 
that $u$ is not invariant. By 5.3 , there exist $x, y$ such that $x \leqq u, \dot{y} \leqq-u$, and $x \sim y \neq 0$. For some $n$ we must have $x u_{n} \neq 0$, and from postulate II we then have that there exists $y^{\prime} \leqq y$ such that $y^{\prime} \sim x u_{n}$. From $5.2, y^{\prime} \leqq u_{n} \leqq u$; but $y^{\prime} \leqq y \leqq-u$, and so $y^{\prime}=o$-that is, $x u_{n}=o$ (from 2.3(4)), a contradiction.

5.5. Definition. For each $x \in E$ we can (from 5.4) define the invariant closure $\bar{x}$ of $x$ to be the smallest invariant element containing $x$. To simplify the printing of complicated expressions, we shall sometimes also write $\mathrm{Cl}(x)$ instead of $\bar{x}$. This closure operation will then have the usual properties familiar in topology $(\bar{o}=o, \quad \bar{e}=e, \quad \mathrm{Cl}(\mathrm{Cl}(x))=\mathrm{Cl}(x) \geqq x, \quad \mathrm{Cl}(x) \vee \mathrm{Cl}(y)$ $=\mathrm{Cl}(x \vee y)$, and $x$ is invariant if and only if $\bar{x}=x)$, as well as some others, notably:

5.6. For any collection of elements $\left\{x_{\alpha}\right\}, \mathrm{Cl}\left(\mathrm{V}_{x_{\alpha}}\right)=\mathrm{V}_{\bar{x}_{\alpha}}$.

For it is trivial that $\mathrm{Cl}\left(\mathrm{V} x_{\alpha}\right) \geqq \mathrm{V}_{\bar{x}_{\alpha}}$, while conversely $\mathrm{V}_{\bar{x}_{\alpha}}$ is invariant (5.4) and greater than or equal to $V x_{\alpha}$, and so greater than or equal to $\mathrm{Cl}\left(\mathrm{V} x_{\alpha}\right)$.

5.7. For any $x, \bar{x}=\bigvee\left\{y \mid y \sim y^{\prime} \leqq x\right\}$.

Let $x^{*}$ denote the element on the right. Any invariant $u \geqq x$ will satisfy $u \geqq x^{*}$ (from 5.2 ), and so $\bar{x} \geqq x^{*}$. All that remains to be proved is that $x^{*}$ is invariant; for clearly $x^{*} \geqq x$. If $x^{*} \notin U$, then (5.3) there exist $z, t$ such that $z \leqq x^{*}, t \leqq-x^{*}, z \sim t$, and $z \neq o$. Hence for some $y \sim y^{\prime} \leqq x$ we have $z y \neq o$; and postulate II(2.1) then gives the existence of $p \leqq t$ and $q \leqq y^{\prime}$ such that $p \sim z y \sim q$. Since $q \leqq x$, this shows that $p \leqq x^{*}$; but $p \leqq t \leqq-x^{*}$, and so $p=o$. That is $(2.3(4)), z y=o$, a contradiction.

5.8. If $x \sim x^{\prime}$, then $\mathrm{Cl}(x)=\mathrm{Cl}\left(x^{\prime}\right)$.

This is immediate from 5.7 and postulate II $(2.1)$.

5.9. $\bar{x} \bar{y}=o \rightleftarrows$ whenever $h, k$ are such that $h \leqq x, k \leqq y$, and $h \sim k$, then $h=k=0$.

The implication $\rightarrow$ is trivial, since $h \leqq \bar{x}$ and also $h \leqq \bar{y}$ (5.7). Conversely, if nonzero $h, k$ exist such that $h \leqq x, k \leqq y$, and $h \sim k$, we have, on writing $u=\bar{h}=\bar{k}($ from 5.8$), u \leqq \bar{x} \bar{y}$.

5.10. If $u$ is invariant, $\mathrm{Cl}(x u)=\mathrm{Cl}(x) u$.

Trivially $\mathrm{Cl}(x u) \leqq \mathrm{Cl}(x) u$. Write $p=\mathrm{Cl}(x) u-\mathrm{Cl}(x u)$, and suppose $p \neq o$. Since $p \leqq \bar{x}, 5.7$ shows that $p y \neq o$ for some $y \sim y^{\prime} \leqq x$. Write $q=p y$; then, by postulate II, there exists $q^{\prime} \leqq x$ such that $q \sim q^{\prime}$. Since $q^{\prime} \sim q \leqq p \leqq u$, 5.2 shows that $q^{\prime} \leqq u$; thus $q^{\prime} \leqq x u$, and so (5.7) $q \leqq \mathrm{Cl}(x u)$. But $q \leqq p$ $\leqq-\mathrm{Cl}(x u)$, so that $q=o$, a contradiction.

6. Bounded elements. In this section we develop further fundamental properties of the invariant and other elements of $(E, \sim)$ which depend on the notion of boundedness. (One example of the way in which this property is of service has already been furnished by the proof of 2.3(4).)

6.1. ("Cancellation law"). If $a, a^{\prime}$ are both bounded and equivalent, and if $b \leqq a, b^{\prime} \leqq a^{\prime}$, and $b \sim b^{\prime}$, then $a-b \sim a^{\prime}-b^{\prime}$.

REMARK. As will be proved later, it suffices that $a$ be bounded; for the 
boundedness of $a^{\prime}$ will then follow from $a^{\prime} \sim a(6.4)$.

From 2.3(5) and 5.9, there exist $x \leqq a-b, y \leqq a^{\prime}-b^{\prime}$, such that (i) $x \sim y$, (ii) on writing $p=(a-b)-x, q=\left(a^{\prime}-b^{\prime}\right)-y$, we have $\bar{p} \bar{q}=o$. We shall prove $p=o$; a similar argument will then show that $q=o$, whence the result will follow.

Write $h=\varnothing a^{\prime}$; thus $h q=o$, and accordingly (since the elements $b^{\prime}, y, q$ are disjoint and $\left.b^{\prime} \vee y \vee q=a^{\prime}\right) \quad h \leqq b^{\prime} \vee y \sim b \vee x$. Thus (postulate II, 2.1) there exists $k \leqq b \bigvee x$ such that $k \sim h$. Since $p \bigvee k \leqq a \sim a^{\prime}$, there exists $m \leqq a^{\prime}$ such that $m \sim p \vee k$. Now, $k \leqq p$ (from 5.2, since $k \sim h \leqq p$ ), and therefore $h \bigvee k \leqq \bar{p}$; and since $\bar{p}$ is invariant, it follows (from 5.2 again) that $m \leqq \bar{p}$. Thus $m \leqq h \sim k$, so (from II again) there exists $n \leqq k$ such that $n \sim m \sim k \bigvee p$. But $k \bigvee p$ is bounded (from 2.3(2), for $a$ is bounded). Hence $n=k \bigvee p$, so that $k \bigvee p \leqq k$; and since $k p=o$ (for $k \leqq b \bigvee x$ ) it follows that $p=o$; Q.E.D.

6.2. Given $y \sim x=\bigvee x_{n}$, where the sequence $\left\{x_{n}\right\}$ is disjoint, there exists a disjoint sequence $\left\{y_{n}\right\}$ such that $y=\bigvee y_{n}$ and $y_{n} \sim x_{n}(n=1,2, \cdots)$.

First assume that $x$ and $y$ are both bounded. In this case, postulate II gives the existence of $y_{1} \leqq y$ such that $y_{1} \sim x_{1}$; and from 6.1 we have $x-x_{1} \sim y-y_{1}$. A straightforward induction, repeating this argument, gives a disjoint sequence of elements $y_{n} \leqq\left(y-\left(y_{1} \bigvee y_{2} \vee \cdots \vee y_{n-1}\right)\right)$ such that $y_{n} \sim x_{n}$. Write $y^{\prime}=\mathrm{V} y_{n}$; thus $y^{\prime} \leqq y$ and (from postulate I) $y^{\prime} \sim x \sim y$. Since $y$ is bounded, we have $y^{\prime}=y$, and the result is established in this case.

In the general case, we apply postulate III (2.1) to write $x=\bigvee f_{n}, y=\bigvee g_{n}$, where $f_{n} \sim g_{n}$ and the sequences $\left\{f_{n}\right\},\left\{g_{n}\right\}$ are each disjoint and bounded. Write $h_{m n}=x_{m} f_{n}$. The case already established enables us to write $g_{n}=\mathrm{V}_{m} k_{m n}$ where $k_{m n} \sim h_{m n}$ and $k_{m n} k_{m^{\prime} n}=o\left(m \neq m^{\prime}\right)$. Define $y_{m}=\bigvee_{n} k_{m n}$; from postulate I we have $y_{m} \sim \bigvee_{n} h_{m n}=x_{m}$; the elements $y_{m}$ are clearly disjoint; and finally $\mathrm{V} y_{m}=\mathrm{V}_{m, n} k_{m n}=\bigvee g_{n}=y$.

6.3. A necessary and sufficient condition that $x$ be unbounded is that there exist an infinite disjoint sequence $\left\{z_{n}\right\}$, with $z_{n} \leqq x, z_{n} \neq 0$, and $z_{1} \sim z_{2}$ $\sim \cdots \sim z_{n} \sim \cdots$.

The sufficiency is trivial, since $\vee\left\{z_{n} \mid n \geqq 1\right\}$ and $\vee\left\{z_{n} \mid n \geqq 2\right\}$ are (from postulate I) equivalent, so that $\mathrm{V}_{z_{n}}$ (and so, a fortiori, $x$ ) is not bounded.

Conversely, suppose $x$ is unbounded; then there exists $z_{1} \leqq x$ such that $z_{1} \neq 0$ and $x \sim x-z_{1}$. The elements $z_{n}$ are now defined by induction. When $z_{n}$ has been defined, in such a way that $z_{n} \sim z_{1}, z_{n} \leqq x-\left(z_{1} \bigvee \cdots \vee z_{n-1}\right)=y_{n-1}$, say, and $y_{n} \sim x$, we apply 6.2 to the decomposition $x=z_{1} \vee y_{1}$ to obtain an "equivalent" decomposition $y_{n}=z_{n+1} \bigvee y_{n+1}$ such that $z_{n+1} y_{n+1}=0, z_{n+1} \sim z_{1}$, and $y_{n+1} \sim y_{1}=x-z_{1} \sim x$. Thus the inductive hypotheses are maintained, and the desired sequence $\left\{z_{n}\right\}$ is thereby constructed.

6.4. If $x \sim x^{\prime}$ and $x$ is bounded, then so is $x^{\prime}$.

This follows from 6.3 and 6.2 , since if $x^{\prime}$ were unbounded, the decomposition $x^{\prime}=\bigvee z_{n} \bigvee\left(x^{\prime}-\bigvee z_{n}\right)$ given by 6.3 could (6.2) be imitated in $x$, contradicting the boundedness of $x$. 
REMARK. From these results one can show that if $x, y$ are both bounded, then so is $x \bigvee y$; we omit the proof, since the result will not be needed.

6.5. Let $\left\{x_{n}\right\}$ be a sequence of bounded elements whose invariant closures are disjoint (that is, $\bar{x}_{m} \bar{x}_{n}=o$ if $m \neq n$ ). Then $\mathrm{V}_{x_{n}}$ is bounded.

Suppose $\mathrm{V} x_{n}=x \sim x-y$ for some $y \leqq x$; we must prove $y=o$. Now, for each $n$ we have $x_{n} \leqq x \sim x-y$; hence (postulate II) there exists $x_{n}^{\prime} \leqq x-y$ such that $x_{n}^{\prime} \sim x_{n}$. Now if $m \neq n$ we have $x_{n}^{\prime} x_{m} \leqq \bar{x}_{n}$ (from 5.7), and also $x_{n}^{\prime} x_{m} \leqq x_{m} \leqq \bar{x}_{m}$; hence $x_{n}^{\prime} x_{m}=o(m \neq n)$. It follows that $x_{n}^{\prime} \leqq x_{n}$, and so (since $x_{n}$ is bounded) $x_{n}^{\prime}=x_{n}$; that is, $x_{n} y=o$ for each $n$, so that $y=x y=0$.

6.6. If $u$ is invariant and $x \sim y$, then $u x \sim u y$.

First suppose $x$ and $y$ are both bounded. Then (postulate II) there exists $z \leqq y$ such that $z \sim u x$. From $5.2, z \leqq u$, so $z \leqq u y$. Similarly there exists $t \leqq u x$ such that $t \sim u y$. By II again, there exists $t^{\prime} \leqq z$ such that $t^{\prime} \sim t \sim u y$. But $u y$ is bounded (2.3(2)), so $t^{\prime} \sim u y$; and consequently $z=u y$, whence the result.

The extension to the general case now follows from postulate III; we have $x=\mathrm{V} x_{n}, y=\mathrm{V} y_{n}$, where each of the sequences $\left\{x_{n}\right\},\left\{y_{n}\right\}$ is disjoint, and where $x_{n}, y_{n}$ are bounded and equivalent. The preceding case gives $u x_{n} \sim u y_{n}$; whence (from postulate I) $u x \sim u y$.

\section{Equivalence classes.}

7.1. Notation. We write $[x]$ for the "equivalence class" $\left\{x^{\prime} \mid x^{\prime} \sim x\right\}$, and write $[x] \leqq[y]$ (or $[y] \geqq[x]$ ) to mean that there exist $x^{\prime} \in[x]$ and $y^{\prime} \in[y]$ such that $x^{\prime} \leqq y^{\prime}$. Thus (from II, 2.1) there will then exist, for each $y^{\prime} \in[y]$, an $x^{\prime} \in[x]$ such that $x^{\prime} \leqq y^{\prime}\left({ }^{17}\right)$. It follows immediately that if $[x] \leqq[y]$ and $[y] \leqq[z]$ then $[x] \leqq[z]$ (so that $\leqq$ is in fact a partial ordering of the equivalence classes), and that if $[x] \leqq[y] \leqq[x]$ and $x$ is bounded then $[x]=[y]$. (This holds even if $x$ is unbounded; but this fact will not be needed.) We further write $[x]<[y]$ (or $[y]>[x]$ ) to mean that $[x] \leqq[y]$ and $[x] \neq[y]\left({ }^{18}\right)$. Of course, two given equivalence classes will in general be incomparable.

Note that $[x] \leqq[y]$ implies $\bar{x} \leqq \bar{y}$ (from 5.8). (The converse is false, in general.)

7.2. If $x_{1} \geqq x_{2} \geqq \cdots$ is a decreasing sequence of bounded elements such that $\left[x_{n}\right] \geqq[y]$ for all $n$, then $\left[\Lambda x_{n}\right] \geqq[y]$.

Since $\left[x_{1}\right] \geqq[y]$, there exist $x_{1}^{\prime} \sim x_{1}, y^{\prime} \sim y$, such that $x_{1}^{\prime} \geqq y^{\prime}$. Now elements $x_{2}^{\prime}, x_{3}^{\prime}, \cdots$, such that $x_{1}^{\prime} \geqq x_{2}^{\prime} \geqq \cdots \geqq y^{\prime}$ and $x_{n}^{\prime} \sim x_{n}$, are defined by induction, as follows. When $x_{n}^{\prime}$ has been defined, postulate II gives the existence of $y^{n+1} \leqq x_{n+1}$ such that $y^{n+1} \sim y \sim y^{\prime}$; and (from 6.4 and 6.1) $x_{n}^{\prime}-y^{\prime}$ $\sim x_{n}-y^{n+1} \geqq x_{n}-x_{n+1}$. Hence (II) there exists $z_{n+1} \leqq x_{n}^{\prime}-y^{\prime}$ such that

(17) There will also exist, for each $x^{\prime} \in[x]$, a $y^{\prime} \in[y]$ such that $x^{\prime} \leqq y^{\prime}$; but the proof is not easy, except in the case in which $y$ is bounded.

(18) One could go on to make $[x]$ play the role of an "abstract measure" $\lambda(x)$, by defining addition, and so on, suitably, along the lines of [12]; cf. also $\$ 12$. We shall not use this procedure, since a much more specific abstract measure will be constructed later. 
$z_{n+1} \sim x_{n}-x_{n+1}$. Define $x_{n+1}^{\prime}=x_{n}^{\prime}-z_{n+1}$; thus $x_{n}^{\prime} \geqq x_{n+1}^{\prime} \geqq y^{\prime}$, and (from 6.1) $x_{n+1}^{\prime} \sim x_{n}-\left(x_{n}-x_{n+1}\right)=x_{n+1}$.

Now $x_{1}^{\prime}-\Lambda x_{n}^{\prime}=\bigvee\left(x_{n}^{\prime}-x_{n+1}^{\prime}\right) \sim \bigvee\left(x_{n}-x_{n+1}\right) \quad($ from I $)=x_{1}-\Lambda x_{n}$; hence (6.1 and 6.4) $\Lambda x_{n} \sim \wedge x_{n}^{\prime} \geqq y^{\prime} \sim y$; and the result is proved.

7.3. If $x_{1} \leqq x_{2} \leqq \cdots$ is an increasing sequence such that $\left[x_{n}\right] \leqq[y]$ for all $n$, and if $y$ is bounded, then $\left[\mathrm{V} x_{n}\right] \leqq[y]$.

This is proved by an argument similar to the preceding $\left({ }^{19}\right)$.

7.4. If $[x] \leqq[y]$, and $u \in U$ (the set of invariant elements), then [ux] $\leqq[u y]$.

This is an immediate consequence of 6.6.

7.5. Given $a, b \in E$, there exist "maximal" equivalent elements $x \leqq a, y \leqq b$, such that (i) $\mathrm{Cl}(a-x), \mathrm{Cl}(b-y)=0$, (ii) given any other equivalent elements $x^{\prime} \leqq a$, $y^{\prime} \leqq b$, we have $\left[x^{\prime}\right] \leqq[x]$ and $\left[y^{\prime}\right] \leqq[y]$.

The existence of $x \leqq a$ and $y \leqq b$ such that $x \sim y$ and (i) holds is given by 2.3(5), in view of 5.9. We prove that they satisfy (ii). Write $z=x \mathrm{Cl}\left(x^{\prime}-x\right)$ and $t=x^{\prime} z \vee\left(x^{\prime}-x\right)$; thus $t \leqq x^{\prime} \mathrm{Cl}\left(x^{\prime}-x\right)$. Since $t \leqq x^{\prime} \sim y^{\prime}$, there exists $s \sim t$ such that $s \leqq y^{\prime} \leqq b$. But $s \leqq \mathrm{Cl} t \leqq \mathrm{Cl}\left(x^{\prime}-x\right) \leqq \mathrm{Cl}(a-x)$, so $s(b-y)=o$, and therefore $s \leqq y$. Thus $s \leqq y \mathrm{Cl}\left(x^{\prime}-x\right) \sim x \mathrm{Cl}\left(x^{\prime}-x\right) \quad$ (from 6.6$)=z$, so that there exists $s^{\prime} \leqq z$ such that $s^{\prime} \sim s \sim t$. From postulate I we now have $x^{\prime}=t \bigvee x^{\prime}(x-z) \sim s^{\prime} \bigvee x^{\prime}(x-z) \leqq x$, and so $\left[x^{\prime}\right] \leqq[x]$. Similarly $\left[y^{\prime}\right] \leqq[y]$.

7.6. Given elements $a, b \in E$, of which at least $a$ is bounded, there exists $u \in U$ such that (i) $[a u] \leqq[b u]$, (ii) if $v$ is a nonzero invariant element disjoint from $u$, then $[a v]>[b v]$.

Let $x, y$ be the elements given by 7.5. Write $u=-\mathrm{Cl}(a-x)$; thus $u$ is invariant (5.4). Further, $u a=u x \sim u y$ (from 6.6) $u b$, establishing (i). Finally, if $v$ is invariant and $u v=o$, we have $v \leqq \mathrm{Cl}(a-x)$ and therefore $v \mathrm{Cl}(b-y)=o$; hence $b v=y v \sim x v \leqq a v$, which shows that $[b v] \leqq[a v]$, and that if $[b v]=[a v]$ then $x v \sim a v$-that is, $v(a-x)=0$, since $a$ is bounded. But this would imply $o=\mathrm{Cl}(v(a-x))=v \mathrm{Cl}(a-x)$ (from 5.10) $=v$. Hence if $v \neq 0$ we must have $[b v]<[a v]$.

\section{Relativization.}

8.1. Given any $a \in E$, it was pointed out in 3.1(c) that the "relative algebra" $(E(a), \sim)$, where $E(a)$ consists of all elements $x \leqq a$, satisfies our postulates. We shall here derive, for later use, some relations between this relative algebra $E(a)$ and $E$.

An element $v \leqq a$ which is invariant in $E(a)$ (that is, in $(E(a), \sim)$ ) will be said to be invariant relative to $a$, and the set of elements invariant relative to $a$ will be denoted by $U_{a}$. For the other notations used in this section, we refer to 1.3 .

8.2. A necessary and sufficient condition that an element $v \leqq a$ be invariant relative to $a$ is that there exist $u \in U$ such that $v=a u$. Further, $u$ can be

${ }^{(19)}$ The restriction of boundedness can be removed in 7.3 , but not in 7.2 -just as in the familiar numerical case. 
chosen so that $u \leqq \bar{a}$; and if this is done, the correspondence between $u$ and $v$ is $1-1$.

The condition is sufficient; for if $v=a u$, where $u \in U$, and if $v \sim v^{\prime} \leqq a$, then $v^{\prime} \leqq u$ (from the invariance of $u$ ), and so $v^{\prime} \leqq v$ that is, $v \in U_{a}$.

The condition is necessary. For if $v \in U_{a}$, define $u=\bar{v}$. Trivially $v \leqq a u$; let $w=a u-v$, and suppose $w \neq o$. Then, since $w \leqq \bar{v}, 5.7$ shows that, for some $x \sim x^{\prime} \leqq v$, we have $w x=y$ (say) $\neq 0$. From II, there exists $y^{\prime} \leqq x^{\prime}$ such that $y \sim y^{\prime}$; and since $y^{\prime} \leqq v$ and $y \leqq w \leqq a, 5.2$ applied to $E(a)$ shows that $y \leqq v$. But $y \leqq w \leqq-v$; hence $y=o$, a contradiction. Thus $v=a u$, where $u=\bar{v}$, so that $u \in U$ and $u \leqq \bar{a}$.

To show that the correspondence is $1-1$, we must show that if $a u_{1}=a u_{2}$ (where $u_{1}, u_{2} \in U(\bar{a})$ ) then $u_{1}=u_{2}$; and, writing $u=u_{1}+{ }_{2} u_{2}$, it will suffice to show that if $a u=o$ (where, from 5.4, $u \in U(\bar{a})$ ) then $u=o$. But we have $o=\mathrm{Cl}(a u)=\mathrm{Cl}(a) u(5.10)=u$.

The result just established can be restated as:

8.3 CoROLlary. The algebra $U_{a}=a U$, and is isomorphic to the principal ideal $U(\bar{a})$ (of all elements of $U$ which are $\leqq \bar{a}$ ) under the correspondence $v \rightarrow u=\bar{v}, u \rightarrow v=a u$.

8.4. An element of $E(a)$ is bounded relative to $a$ (that is, in $E(a)$ ) if and only if it is bounded.

This is trivial from the definition of boundedness.

8.5. For any set $P \subset E$, the Borel field relative to a generated by aP (in symbols, $\left.\mathbb{B}_{a}(a P)\right)$ is $a \mathrm{~B}(P)$.

Write $\mathcal{B}^{\prime}=a \mathcal{B}(P)=$ set of all elements $a x$, where $x \in \mathcal{B}(P)$. Clearly $\mathbb{B}^{\prime} \supset a P$; and if $y$ and $y_{n}$ belong to $\mathcal{B}^{\prime}$, then so do $a-y$ and $V_{y_{n}}$; hence, $\mathcal{B}^{\prime} \supset B_{a}(a P)$. Now write $\mathbb{B}^{\prime \prime}=\mathbb{B}_{a}(a P) \bigvee E(-a)=$ set of all elements expressible as $y \bigvee z$ where $y \in \mathscr{B}_{a}(a P)$ and $z \in E(-a)$. It is easy to see that $\mathbb{B}^{\prime \prime} \supset P$, and that if $x$ and $x_{n} \in \mathbb{B}^{\prime \prime}$ then so do $-x$ and $\bigvee x_{n}$. Hence $\mathbb{B}^{\prime \prime} \supset \mathbb{B}(P)$; so $a \mathscr{B}(P) \subset a \mathbb{B}^{\prime \prime}=\mathscr{B}_{a}(a P)$, completing the proof.

\section{Indecomposable elements.}

9.1. Definitions. An element $x$ is decomposable if there exist $y_{1}, y_{2}$ such that $o<y_{i} \leqq x(i=1,2), y_{1} \sim y_{2}$, and $y_{1} y_{2}=o$. Otherwise $x$ is said to be indecomposable. Clearly $o$ is indecomposable, and so is each atom of $E$; there may, of course, be other indecomposable elements. The indecomposable elements are the easiest to deal with, and we shall dispose of them in this section; this will in effect reduce the problem of representing $E$ to the case in which all nonzero elements are decomposable. tion.

9.2. The following properties are easy consequences of the above defini-

(1) If $x$ is indecomposable, and $y \leqq x$, then $y$ is indecomposable.

(2) Indecomposable elements are bounded. (From 6.3.)

(3) If $x \sim x^{\prime}$ and $x$ is indecomposable, then so is $x^{\prime}$. (From 6.2.) 
(4) If $x$ is indecomposable, and $y \leqq x$, then $y=x \bar{y}$.

For trivially $y \in x \bar{y}$. Write $z=x \bar{y}-y$; if $z \neq 0,5.7$ shows that $z y^{\prime} \neq 0$ for some $y^{\prime} \sim y^{\prime \prime} \leqq y$, and then II gives the existence of $z_{1} \leqq z$ and $y_{1} \leqq y$ such that $z_{1} \sim z y^{\prime} \sim y_{1}$. The definition of indecomposability gives $z_{1}=o$, whence $z y^{\prime}=o$, a contradiction.

(5) If $x$ is indecomposable and $y_{1} \bigvee y_{2} \leqq x$, and if $y_{1} \sim y_{2}$, then $y_{1}=y_{2}$.

For, from (4) and 5.8, $y_{1}=x \bar{y}_{1}=x \bar{y}_{2}=y_{2}$.

(6) If $x$ is indecomposable, $y \bigvee z \leqq x$, and $y z=o$, then $\bar{y} \bar{z}=o$. (From 5.9.)

9.3. Let $\left\{x_{n}\right\}$ be a sequence of indecomposable elements such that $\bar{x}_{m} \bar{x}_{n}=o$ whenever $m \neq n$. Then $V_{x_{n}}$ is indecomposable.

Let $y \bigvee z \leqq \bigvee x_{n}$, and suppose $y \sim z$ and $y z=o$. (We must prove $y=o$.) Let $y_{n}=y x_{n}$; thus the elements $y_{n}$ are disjoint, and $\bigvee y_{n}=y$. Hence (6.2) we can write $z=\bigvee z_{n}^{\prime}$, where the elements $z_{n}^{\prime}$ are disjoint and $z_{n}^{\prime} \sim y_{n}$. Let $z_{n m}=z_{n}^{\prime} x_{m}$; from 6.2 again we can write $y_{n}=\bigvee_{m} y_{n m}$, where $y_{n m} \sim z_{n m}$. Now, if $m \neq n$, we have $y_{n m} \leqq \bar{x}_{m}$ (from 5.7, since $y_{m n} \sim z_{m n} \leqq x_{m}$ ), and so $y_{n m} \leqq x_{n} \bar{x}_{m}=o$. And if $m=n, y_{n m} \bigvee z_{n m} \leqq x_{m}$, which is indecomposable; hence again $y_{n m}=o$. Hence $y=\bigvee_{m, n} y_{n m}=o$.

9.4. If $b_{1} \leqq b_{2} \leqq \cdots$ is an increasing sequence of indecomposable elements, then $\mathrm{V} b_{n}$ is indecomposable.

Write $x_{1}=b_{1}, x_{n}=b_{n}-b_{n-1}$; thus $x_{n}$ is indecomposable (9.2(1)), and $x_{m} x_{n}=o$ if $m \neq n$. Since $x_{m} \bigvee x_{n} \leqq b_{m+n}$, which is indecomposable, 9.2(6) shows that $\bar{x}_{m} \bar{x}_{n}=o$. So $\mathrm{V} x_{n}$ is indecomposable (9.3); and $\mathrm{V} x_{n}=\mathrm{V} b_{n}$.

9.5. Given any collection $C$ of indecomposable elements, there exists an indecomposable element $b$ such that (i) $b \leqq \bigvee\{c \mid c \in C\}$, (ii) for each $c \in C,[c] \leqq[b]$.

By transfinite induction, using 9.4, we see that there is a maximal indecomposable $b$ satisfying (i). We prove that $b$ satisfies (ii) also. In fact, given $c \in C$, there exist (7.5) elements $b_{1} \leqq b, c_{1} \leqq c$, such that $b_{1} \sim c_{1}$ and $\mathrm{Cl}\left(c-c_{1}\right)$ . $\mathrm{Cl}\left(b-b_{1}\right)=o$. Also $(9.2(6)) \mathrm{Cl}\left(c_{1}\right) \mathrm{Cl}\left(c-c_{1}\right)=o$, and $\bar{b}_{1}=\bar{c}_{1}(5.8)$, so that $\mathrm{Cl}\left(b_{1}\right) \mathrm{Cl}\left(c-c_{1}\right)=o$. Again, from 9.2(6), we have $\mathrm{Cl}\left(b_{1}\right) \mathrm{Cl}\left(b-b_{1}\right)=o$. Hence 9.3 shows that the element $p=\mathrm{Cl}\left(b_{1}\right) \vee \mathrm{Cl}\left(b-b_{1}\right) \vee \mathrm{Cl}\left(c-c_{1}\right)$ is indecomposable. But $b \leqq p \leqq \bigvee\{c \mid c \in C\}$. Hence $b=p$, proving that $c-c_{1}=o$, that is, that $c \sim b_{1} \leqq b, Q$.E.D.

9.6. Notation. Let $e^{o}$ denote the supremum of all the indecomposable elements. (Naturally, $e^{o}$ may be $o$, and also need not itself be indecomposable.)

THEOREM. There exists a disjoint sequence $\left\{b_{n}\right\}$ of indecomposable elements, such that (i) $\mathrm{V} b_{n}=e^{o}$, and (ii) for each $m, n$, either $\left[b_{m}\right] \leqq\left[b_{n}\right]$ or $\left[b_{n}\right]$ $\leqq\left[b_{m}\right]$. Further, (iii) $e^{o}$ is invariant.

We first define a transfinite sequence $\left\{b_{\alpha}\right\}$ of disjoint indecomposable elements, as follows. From 9.5 applied to the collection $C_{1}$ of all indecomposable elements, there exists an indecomposable element $b_{1} \leqq e^{o}$ such that $[c] \leqq\left[b_{1}\right]$ for every indecomposable $c$. When $b_{\beta}$ has been defined for all $\beta<\alpha$, we define $C_{\alpha}=$ set of all indecomposable elements $c$ satisfying $c \leqq e^{o}-\mathrm{V}_{\beta<\alpha} b_{\beta}$. By 9.5, 
there exists an indecomposable $b_{\alpha} \leqq e^{o}-\bigvee_{\beta<\alpha} b_{\beta}$ such that $[c] \leqq\left[b_{\alpha}\right]$ for every $c \in C_{\alpha}$. From postulate $0(2.1)$ we have $b_{\alpha_{0}}=o$ for some $\alpha_{0}<\omega_{1}$, and the process is terminated. $C_{\alpha_{0}}$ must then consist of $o$ only, so that the element $d=e^{o}$ $-\mathrm{V}_{\alpha<\alpha_{0}} b_{\alpha}$ must be $o$ (for otherwise, since $d \leqq e^{o}$, there would exist an indecomposable $c$ such that $c d \neq o$; and, from 9.2(1), $c d \in C_{\alpha_{0}}$ ). On renumbering the elements $b_{\alpha}\left(\alpha<\alpha_{0}\right)$ into a simple sequence, we see that (i) and (ii) are satisfied.

To prove (iii), suppose $y \sim x \leqq e^{o}$; it will suffice to prove $y \leqq e^{o}$. From (i), $x=\mathrm{V} x b_{n}$; hence (6.2) we can write $y=\mathrm{V} y_{n}$ where $y_{n} \sim x b_{n}$. But $x b_{n}$ is indecomposable, since $b_{n}$ is $(9.2(1))$; hence so is $y_{n}(9.2(3))$, so that $y_{n} \leqq e^{o}$ for each $n$. It follows that $y \leqq e^{o}$.

9.7. Let $K$ (as in $3.1(a)$ ) be the numerical measure algebra generated by $\aleph_{0}$ atoms $p_{n}(n=1,2, \cdots)$ each of measure 1 ; that is, the elements of $K$ are the subsets of $U\left(p_{n}\right)$, and the measure of any such set is the number of elements $p_{n}$ in it.

TheOREm. The relative algebra $\left(E\left(e^{o}\right), \sim\right)$ is isomorphic to a principal ideal in the direct product $K \otimes U\left(e^{o}\right)$.

(Here $U\left(e^{o}\right)$ denotes, as usual, the principal ideal in $U$ of $e^{o-w h i c h ~ b e-~}$ longs to $U$, as just shown.)

Let $R, S$ denote the representation spaces of $K, U\left(e^{o}\right)$, respectively (cf. $\S 4)$. The open-closed subset $p_{n}^{*}$ of $R$ which corresponds to $p_{n} \in K$ is now easily seen to consist of a single point (say) $q_{n}$. Write $Q=R-U\left(q_{n}\right)$; thus (from 4.2(2)) $Q$ is of the first category in $R$.

Now let $b_{n}$ be the sequence of indecomposable elements given by 9.6. For each $x \leqq e^{o}$, define $\psi(x)$ to be the subset $\cup_{n=1}^{\infty}\left(q_{n},\left(\mathrm{Cl}\left(x b_{n}\right)\right)^{*}\right)$ of $T=R \times S$; clearly $\psi(x) \in \mathcal{B}_{r}(T)$. And define $\theta(x)$ to be the element $\{\psi(x)\}$ of $K \otimes U\left(e^{o}\right)$, that is, the class of all sets $H \in \mathcal{B}_{r}(T)$ such that $M\left\{H+{ }_{2} \psi(x)\right\}$ is identically $0, M$ being the continuous-function-valued "measure" defined in $\$ 4$. We shall show that $\theta$ is the desired isomorphism.

(1) For any sequence $\left\{x_{n}\right\}$ with $x_{n} \leqq e^{o}, \theta\left(\mathrm{V} x_{n}\right)=\mathrm{V} \theta\left(x_{n}\right)$.

For we have $\mathrm{Cl}\left(\mathrm{V}_{m} x_{m} b_{n}\right)=\mathrm{V}_{m} \mathrm{Cl}\left(x_{m} b_{n}\right)(5.6)$, and so (4.2(2)) $\left(\mathrm{Cl}\left(\mathrm{V}_{m} x_{m} b_{n}\right)\right)^{*}$ $=\mathrm{U}_{m}\left(\mathrm{Cl}\left(x_{m} b_{n}\right)\right)^{*}+{ }_{2} Y_{n}$, where $Y_{n}$ is of the first category in $S$. Hence $\psi\left(\mathrm{V} x_{m}\right)$ $=\mathrm{U} \psi\left(x_{m}\right)+{ }_{2} \mathrm{U}\left(q_{n}, Y_{n}\right)$, so that (the sets $\left(q_{n}, Y_{n}\right)$ being null) $\theta\left(\mathrm{V} x_{m}\right)=\mathrm{V} \theta\left(x_{m}\right)$.

(2) If $x \leqq e^{o}, \theta\left(e^{o}-x\right)=\theta\left(e^{o}\right)-\theta(x)$.

For we have $\mathrm{Cl}\left(\left(e^{o}-x\right) b_{n}\right) \quad \mathrm{Cl}\left(x b_{n}\right)=o$ from $9.2(6)$; and trivially $\mathrm{Cl}\left(\left(e^{o}-x\right) b_{n}\right) \vee \mathrm{Cl}\left(x b_{n}\right)=\mathrm{Cl}\left(b_{n}\right)$. Hence

$$
\begin{aligned}
\psi\left(e^{o}\right)-\psi(x) & =\bigcup\left(q_{n},\left(\mathrm{Cl}\left(b_{n}\right)\right)^{*}\right)-U\left(q_{n},\left(\mathrm{Cl}\left(x b_{n}\right)\right)^{*}\right) \\
& =\bigcup\left(q_{n},\left(\mathrm{Cl}\left(\left(e^{o}-x\right) b_{n}\right)\right)^{*}\right)=\psi\left(e^{o}-x\right),
\end{aligned}
$$

and (2) follows.

(3) If $x, y \leqq e^{o}$ and $\theta(x)=\theta(y)$, then $x=y$. 
From (1) and (2), it will suffice to prove (on writing $z=x+{ }_{2} y$ ) that if $\theta(z)=o$ then $z=o$. We are given, then, that $M\left\{\mathrm{U}\left(q_{n},\left(\mathrm{Cl}\left(z b_{n}\right)\right)^{*}\right)\right\}$ is identically 0 ; hence, for each $n, M\left\{\left(q_{n},\left(\mathrm{Cl}\left(z b_{n}\right)\right)^{*}\right)\right\}=0$, and since this function is 1 for each $s \in\left(\mathrm{Cl}\left(z b_{n}\right)\right)^{*}$, it follows that $\left(\mathrm{Cl}\left(z b_{n}\right)\right)^{*}=0$. That is, $\mathrm{Cl}\left(z b_{n}\right)=o$ for each $n$. Thus $z=\mathrm{V}_{z} b_{n}=0$.

(4) If $\{H\} \in K \otimes U\left(e^{o}\right)$ and $\{H\} \leqq \theta\left(e^{o}\right)$, there exists $x \leqq e^{o}$ such that $\theta(x)=\{H\}$.

We first note that, given any $H \in \mathcal{B}_{r}(T)$, there exists $H^{\prime} \in\{H\}$ and expressible as $H^{\prime}=\mathrm{U}\left(q_{n}, u_{n}^{*}\right)\left(u_{n}^{*}\right.$ open-closed in $\left.S\right)$. (This is substantially the result of 4.6 in the present case, and follows by the same reasoning applied here.) Now if $\{H\} \leqq \theta\left(e^{o}\right)=\left\{U\left(q_{n}, b_{n}^{*}\right)\right\}$, the corresponding $H^{\prime}$ must satisfy $u_{n} \leqq \bar{b}_{n}$ for each $n$. Define $x=\mathrm{V} b_{n} u_{n}$. Then $x \leqq e^{o}$, and (since the elements $b_{n}$ are disjoint) we have $\mathrm{Cl}\left(x b_{n}\right)=\mathrm{Cl}\left(b_{n} u_{n}\right)=\left(\mathrm{Cl}\left(b_{n}\right)\right) u_{n} \quad(5.10)=u_{n}$. Thus $\psi(x)$ $=H^{\prime}$, so that $\theta(x)=\{H\}$.

Properties (1)-(4) show that $\theta$ is an algebraic isomorphism between $E\left(e^{o}\right)$ and the principal ideal of $\theta\left(e^{o}\right)$ in $K \otimes U\left(e^{o}\right)$. All that remains is to show that $\theta$ is equivalence-preserving (both ways).

(5) If $x, y \leqq e^{o}$ and $x \sim y, M\{\theta(x)\}=M\{\theta(y)\}$.

First suppose $x \leqq b_{n}, y \leqq b_{m}$. We have $\bar{x}=\bar{y}=u$, say (5.8); and clearly $\psi(x)=U\left(q_{k}, u_{k}^{*}\right)$ where $u_{k}=o$ if $k \neq n$, and $u_{n}=u$. Hence, from the definition of $M, M\{\theta(x)\}=M\{\psi(x)\}=0$ for $s \notin u$, and 1 for $s \in u$. The same applies to $M\{\theta(y)\}$, which thus equals $M\{\theta(x)\}$.

In the general case, 6.2 shows that we can write $y=V_{y_{n}}$, where the sequence $y_{n}$ is disjoint and $y_{n} \sim x b_{n}$. Now write $y_{n m}=y_{n} b_{m}$; by 6.2 again we can write $x b_{n}=\mathrm{V}_{m} x_{n m}$ where the elements $x_{n m}$ are disjoint and $x_{n m} \sim y_{n m}$. Since $y_{n m} \leqq b_{m}$ and $x_{n m} \leqq b_{n}$, the case already established shows that $M\left\{\theta\left(x_{n m}\right)\right\}$ $=M\left\{\theta\left(y_{n m}\right)\right\}$. But $\mathrm{V}_{m, n} x_{m n}=x$, and $\mathrm{V}_{m, n} y_{m n}=y$; hence, from the countable additivity of $M, M\{\theta(x)\}=M\{\theta(y)\}$.

(6) If $x, y \leqq e^{o}$ and $M\{\theta(x)\}=M\{\theta(y)\}$, then $x \sim y$.

First suppose $\theta(x) \leqq\left\{\left(q_{n}, \bar{b}_{n}^{*}\right)\right\}$ and $\theta(y) \leqq\left\{\left(q_{m}, \bar{b}_{m}^{*}\right)\right\}$. Then $x b_{k}=o$ unless $k=n$, so that $x \leqq b_{n}$; and similarly $y \leqq b_{m}$. The equality of the measure functions now gives $\bar{x}=\bar{y}$. Now (9.6) we can assume without loss of generality that $\left[b_{n}\right] \leqq\left[b_{m}\right]$; and, on applying postulate II, we obtain $x \sim x^{\prime} \leqq b_{m}$. Hence $x^{\prime}=\bar{x}^{\prime} b_{m}$ (from 9.2(4)) $=\bar{x} b_{m}$ (from 5.8) $=\bar{y} b_{m}=y$ (from 9.2(4) again). That is, $y=x^{\prime} \sim x$.

The extension to the general case now follows by an argument similar to that used in proving (5). (We apply 6.2 to $K \otimes U\left(e^{o}\right)$.) The proof of the theorem is thus complete.

Remark. In the present case, the direct product (as constructed in \$4) can be simplified. It is not hard to see that the abstract measure-algebra $K \otimes U\left(e^{o}\right)$ is isomorphic with the algebra of sequences $\left\{u_{n}\right\}\left(u_{n} \in U\left(e^{o}\right)\right)$, in which the algebraic operations are defined "coordinatewise" (cf. 3.1(d)), but in which the equivalence relation is induced by the continuous numerical 
function $M\left\{u_{n}\right\}$ on $S$, defined by: the value of $M\left\{u_{n}\right\}$ at $s \in S$ is (except for a first category set of $s$ 's) the number of values of $n$ for which $s \in u_{n}^{*}$.

\section{Reduction to the bounded case.}

10.1. The ideal $E\left(e^{o}\right)$ having been disposed of by the theorem just established, we next study the ideal (that is, relative algebra) $E\left(-e^{\circ}\right)$, in which (from the definition of $e^{o}$ ) no nonzero element can be indecomposable. In effect this means that the indecomposable elements have been eliminated. In the present section we shall make a further reduction which will in effect eliminate the unbounded elements.

10.2. Definitions. We write $e^{\prime}$ to denote the supremum of all bounded invariant elements in $E\left(-e^{o}\right)$, and write $e^{\prime \prime}=\left(-e^{o}\right)-e^{\prime}$. Thus $e^{o}, e^{\prime}$ and $e^{\prime \prime}$ are disjoint, and $e^{\circ} \bigvee e^{\prime} \bigvee e^{\prime \prime}=e$.

\subsection{Theorem. $e^{\prime}$ is bounded and invariant.}

That $e^{\prime}$ is invariant follows from 5.4. Further, the countable chain condition (postulate 0 ) gives $e^{\prime}=\mathrm{V} u_{n}$ for some sequence $\left\{u_{n}\right\}$ of bounded invariant elements. Define $v_{1}=u_{1}, v_{n}=u_{n}-\bigvee_{1}^{n-1} u_{i}$; thus $e^{\prime}=\bigvee v_{n}$ where each $v_{n}$ is bounded $(2.3(2))$ and invariant (5.4), and where the sequence $\left\{v_{n}\right\}$ is disjoint. Hence $e^{\prime}$ is bounded, from 6.5.

10.4. Lemma. There exists a sequence $\left\{z_{n}\right\}(n=1,2, \cdots$ to $\infty)$ of disjoint equivalent bounded elements such that $e^{\prime \prime}=\bar{z}_{n}$ for each $n$.

We can obviously assume $e^{\prime \prime} \neq 0$. Then since $e^{\prime \prime}$ is invariant (from 9.6(iii) and 10.3) and disjoint from $e^{\prime}, e^{\prime \prime}$ is unbounded, and consequently (6.3) there exists an infinite sequence of disjoint equivalent nonzero elements (say) $t_{n} \leqq e^{\prime \prime}$. From postulate III (2.1) there exists $p_{11} \leqq t_{1}$ such that $p_{11}$ is bounded and nonzero; and then from postulate II there exists $p_{1 n} \leqq t_{n}$ such that $p_{1 n} \sim p_{11}$. Thus the elements $p_{1 n}$ are disjoint, equivalent, bounded and nonzero, and $\mathrm{V}_{p_{1 n}} \leqq e^{\prime \prime}$. Write $q_{2}=\mathrm{Cl}\left(\mathrm{V}_{p_{1 n}}\right) \leqq e^{\prime \prime}$; thus $e^{\prime \prime}-q_{2}$ is invariant and therefore unbounded (unless it is $o$ ). Thus we obtain (by repeating the argument) a disjoint sequence $\left\{p_{2 n}\right\}$ of bounded equivalent nonzero elements less than or equal to $e^{\prime \prime}-q_{2}$. The argument is repeated transfinitely; when $p_{\beta n}$ has been defined for all $n$ and all $\beta<\alpha$, we set $q_{\alpha}=\mathrm{Cl}\left(\mathrm{V}_{p_{\beta n}}\right)(\beta<\alpha, n=1,2, \cdots)$; if $e^{\prime \prime}-q_{\alpha}$ is not $o$, it is invariant and unbounded, and so there exists a disjoint infinite sequence $\left\{p_{\alpha_{n}}\right\}$ of bounded equivalent nonzero elements less than or equal to $e^{\prime \prime}-q_{\alpha}$. The process must terminate countably; and, on defining $z_{n}=\mathrm{V}_{\alpha} p_{\alpha n}$, the elements $z_{n}$ are disjoint, equivalent (postulate I), bounded (6.5), and satisfy $\mathrm{Cl}\left(\mathrm{V}_{n}\right)=e^{\prime \prime}$. But $\mathrm{Cl}\left(\mathrm{V}_{n}\right)=\operatorname{VCl}\left(z_{n}\right) \quad$ (5.6) $=\mathrm{Cl}\left(z_{n}\right)$ for each $n$ (from 5.8).

10.5. Lemma. Given $u \in U$ such that $o<u \leqq e^{\prime \prime}$, there exists $v \in U$ such that $o<v \leqq u$ and $v=V s_{n}$, where the elements $s_{n}$ are disjoint, equivalent, and bounded $(n=1,2, \cdots$, to $\infty)$. 
Let $\left\{z_{n}\right\}$ be the sequence given by 10.4 . Then the elements $u z_{n}$ are disjoint, equivalent (6.6) and bounded; also they are nonzero, since $\mathrm{Cl}\left(u z_{n}\right)$ $=u \mathrm{Cl}\left(z_{n}\right)(5.10)=u \neq o$. The collection $\left\{u z_{n}\right\}$ can thus be extended to a maximal collection of disjoint equivalent (and thus also bounded and nonzero) elements less than or equal to $u$; and this collection (from postulate 0 ) will consist of exactly $\aleph_{0}$ elements (say) $t_{n}(n=1,2, \cdots$, to $\infty)$. Let $y_{1}, y^{\prime}$ be maximal equivalent elements such that $y_{1} \leqq t_{1}$ and $y^{\prime} \leqq u-\vee t_{n}$, so that (7.5) $\mathrm{Cl}\left(t_{1}-y_{1}\right) \mathrm{Cl}\left(u-\mathrm{V}_{n}-y^{\prime}\right)=o$. Define $v=\mathrm{Cl}\left(t_{1}-y_{1}\right)$; we shall show that $v$ has the required properties.

Trivially, $v$ is invariant and $v \leqq \bar{t}_{1} \leqq u$. Also, $v \neq o$, since otherwise $t_{1}=y_{1} \sim y^{\prime}$, contradicting the maximality of the collection $\left\{t_{n}\right\}$ (for $y^{\prime}$ could then be adjoined to it).

Now, from postulate II, there exists $y_{n} \leqq t_{n}$ such that $y_{n} \sim y_{1}$, and (since then $\left.y_{n} \sim y^{\prime}\right)$ there exists $x_{n} \leqq y_{n}$ such that $x_{n} \sim y^{\prime} v$. Define $s_{1}=y^{\prime} v \vee\left(t_{1}-y_{1}\right)$ and (if $n>1) s_{n}=x_{n-1} \vee\left(t_{n}-y_{n}\right)$; thus the elements $s_{n}(n=1,2, \cdots$ to $\infty)$ are disjoint and equivalent (from 6.1 and postulate III). Further, $s_{n} \sim x_{n}$ $\bigvee\left(t_{n}-y_{n}\right) \leqq t_{n}$, so that $s_{n}$ is bounded (6.4). We have $t_{n}-y_{n} \leqq v$, from 5.7, and $x_{n} \leqq v$, from 5.2 ; thus $\mathrm{V}_{s_{n}} \leqq v$. On the other hand, $v\left(u-\bigvee t_{n}-y^{\prime}\right)=o$, so that $v \leqq \bigvee t_{n} \bigvee y^{\prime}$, and therefore $v \leqq y^{\prime} v \bigvee \bigvee\left\{\left(t_{n}-y_{n}\right) \bigvee\left(y_{n}-x_{n}\right) \bigvee x_{n}\right\} v$; but $\left(y_{n}-x_{n}\right) v$ $\sim\left(y^{\prime}-y^{\prime} v\right) v$ (from 6.6$)=0$, and it follows that $v \leqq \bigvee s_{n}$. Thus $v=\bigvee s_{n}$, as required.

10.6. Theorem. We can write $e^{\prime \prime}=\mathrm{V}^{n}(n=1,2, \cdots$ to $\infty)$, where the elements $f^{n}$ are disjoint, equivalent, bounded, and satisfy $\mathrm{Cl}\left(f^{n}\right)=e^{\prime \prime}$ for each $n$.

Let $\left\{v_{m}\right\}$ be a maximal disjoint (and thus countable) collection of nonzero invariant elements less than or equal to $e^{\prime \prime}$, each of which can be written as $v_{m}=V_{s_{m n}}(n=1,2, \cdots$ to $\infty)$, where the elements $s_{m n}$ are, for each $m$, disjoint, equivalent and bounded. Let $u=e^{\prime \prime}-V v_{m}$; then $u$ is invariant (5.4), so that if $u \neq 0$ an application of 10.5 would at once contradict the maximality of the collection $v_{m}$. Hence $\mathrm{V}_{m, n} s_{m n}=\mathrm{V}_{v_{m}}=e^{\prime \prime}$. Now define $f^{n}=\mathrm{V}_{m} s_{m n}$; thus $V_{f^{n}}=e^{\prime \prime}$, and the elements $f^{n}$ are disjoint, equivalent (postulate I) and bounded (from 6.5). Lastly, $e^{\prime \prime}=\operatorname{VCl}\left(f^{n}\right)=\mathrm{Cl}\left(f^{n}\right)$ for each $n$, from 5.8.

COROLlaRY. For each $n$, the algebra $U_{f^{n}}$ of invariant elements relative to $f^{n}$ is isomorphic to the ideal $U\left(e^{\prime \prime}\right)$.

(From 8.3.)

10.7. Convention. The considerations in what follows (till §19) are going to be applied to the relative abstract measure algebras $E\left(e^{\prime}\right)$ and $E\left(f^{n}\right)$. Thus we shall assume, throughout $\$ \$ 11-18$, that all elements (except o) are decomposable), and that all elements are bounded.

11. Decomposition into homogeneous parts.

11.1 Before the desired direct product representation can be obtained, two further reductions are necessary. In this section we carry out the first 
of these, which will have the effect of enabling us to assume that $E$ is, in a sense to be defined, "homogeneous" with respect to its invariant elements.

Definitions. Given $x \in E$, and given any nonempty subset $P \subset E$, consider all sets $Q \subset E$ such that $E(x) \subset x B(P \cup Q)$ - so that (8.5) $E(x)$ $=x \mathbb{B}(P \cup Q)=\mathbb{B}_{x}(x P \cup x Q)$. The smallest cardinal $\mathfrak{m}$ of such a $Q$ is called the order of $x$ over $P$. (Thus, for example, the order of $o$ over any $P$ is 0 .) If $x \neq 0$, and every $y$ such that $o<y \leqq x$ has order $\mathfrak{m}$ over $P, x$ is said to be homogeneous of order $\mathfrak{m}$ over $P$. Clearly, if $o<y \leqq x$ and $x$ is homogeneous of order $\mathfrak{m}$ over $P$, then so is $y$.

11.2. If $x$ has order $m$ over $P$, and $y \leqq x$, then the order of $y$ over $P$ is not greater than $\mathrm{m}$.

For $E(x)=x \mathbb{B}(P \cup Q)$, where $|Q|=\mathrm{m}$. Given $z \leqq y$, we have $z \in E(x)$, and so $z=x t$ where $t \in \mathbb{B}(P \cup Q)$. Hence $z=y t$; and thus $E(y) \subset y \mathcal{B}(P \cup Q)$.

11.3. If $x$ is homogeneous of order $\mathfrak{m}$ over $P$, where $\mathfrak{m}$ is infinite, and if $a \geqq x$, then $x$ is homogeneous of order $\mathfrak{m}$ over $a P$, and further there exists a set $Q \subset E(x)$, with $|Q|=\mathfrak{m}$, such that $E(x)=x \mathcal{B}(Q \cup a P)$.

We have $E(x)=x B\left(P \cup Q^{\prime}\right)$ for some $Q^{\prime} \subset E$ with $\left|Q^{\prime}\right|=m$. Write $Q=x Q^{\prime}$; then $x \mathscr{B}\left(P \cup Q^{\prime}\right)=x \mathbb{B}(Q \cup a P)$, by 8.5 , since both equal $\mathcal{B}_{x}(Q \cup x P)$. This proves the last part of the statement, and shows that $x$ has order not greater than $m$ over $a P$. To complete the proof, it will suffice to show that if $o<y \leqq x$ than the order of $y$ over $a P$ is not less than $m$. Suppose not; then there exists $Q^{\prime \prime} \subset E$, with $\left|Q^{\prime \prime}\right|<\mathfrak{m}$, such that $E(y)=y \mathcal{B}\left(Q^{\prime \prime} \cup a P\right) \subset y \mathcal{B}\left(Q^{\prime \prime} \cup P \cup(a)\right)$. The homogeneity of $x$ over $P$ now gives $\left|Q^{\prime \prime} \cup(a)\right| \geqq \mathfrak{m}$; and since $\mathfrak{m}$ is infinite this is a contradiction.

11.4. If $\mathfrak{m}$ is an infinite cardinal, and if $x_{n}$ is homogeneous of order $\mathfrak{m}$ over $P(n=1,2, \cdots)$, then so is $\mathrm{V}_{x_{n}}$.

Let $\mathrm{V} x_{n}=x$; we must prove (i) $x$ is of order not greater than $\mathfrak{m}$ over $P$, and (ii) if $o<y \leqq x, y$ is of order not less than $m$ over $P$.

We have $E\left(x_{n}\right)=x_{n} B\left(P \cup Q_{n}\right)$ where $\left|Q_{n}\right|=m$. Let $\cup Q_{n} \cup \cup\left(x_{n}\right)=Q$; thus $|Q|=\mathfrak{m}$ also. Given $y \leqq x$, write $y_{n}=y x_{n} \in E\left(x_{n}\right)$; thus $y_{n}=x_{n} z_{n}$ where $z_{n} \in \mathbb{B}\left(P \cup Q_{n}\right) \subset \mathcal{B}(P \cup Q)$. Since $x_{n} \in Q \subset \mathcal{B}(P \cup Q)$, this gives that each $y_{n}$, and thus also $y=\vee y_{n}$, belongs to $\mathbb{B}(P \cup Q)$, which proves (i).

For (ii), we again write $y_{n}=y x_{n}$; then for some $n$ we have $y_{n} \neq 0$. If $y$ has order less than $\mathfrak{m}$ over $P$, then so does $y_{n}(11.2)$; but this contradicts the homogeneity of $x_{n}$.

11.5. If $x$ is a nonzero element of finite order over $P$, there exists a nonzero element $y \leqq x$ of order 0 over $P$.

We have (say) $E(x)=x \mathbb{B}\left\{P \cup\left(a_{1}\right) \cup\left(a_{2}\right) \cup \cdots \cup\left(a_{n}\right)\right\}$. Consider the $2^{n}$ elements of the form $b_{1} b_{2} \cdots b_{n}$, where each $b_{i}$ is either $a_{i}$ or $-a_{i}$, and all combinations are taken; enumerate them as $t_{1}, t_{2}, \cdots, t_{N}\left(N=2^{n}\right)$. Let $\mathbb{B}^{\prime}$ denote the set of all elements expressible as $\mathrm{V}_{1}^{N} x_{j} t_{j}\left(x_{j} \in \mathcal{B}(P)\right)$. It is easy to verify that $\mathbb{B}^{\prime}$ contains, and thus coincides with, $\mathbb{B}\left\{P \cup\left(a_{1}\right) \cup \cdots \cup\left(a_{n}\right)\right\}$. Thus $E(x)=x \mathbb{B}^{\prime}$. so for at least one $j$ we have $x t_{j}=y$, say, $\neq o$. Now if $z \in E(y)$, 
we have $z \in E(x)=x \mathcal{B}^{\prime}$, so $z=\bigvee_{1}^{N} x_{i} t_{i} x\left(x_{i} \in \mathcal{B}(P)\right)$; but $z \leqq y \leqq t_{j}$, and $t_{i} t_{j}=0$ unless $i=j$. Hence $z=x_{j} t_{j} x=x_{j} y$; and this proves that $E(y) \subset y B(P)$ - that is, $y$ is of order 0 over $P$.

11.6. If $y$ is of order 0 over $U$, then $y$ is indecomposable and therefore zero.

If $y$ were decomposable, there would exist (9.1) $z_{1}, z_{2} \leqq y$ such that $z_{1} \sim z_{2}$, $z_{1} z_{2}=o$, and $z_{i} \neq o(i=1,2)$. Since $E(y)=y U$, by hypothesis, we have $z_{i}=y u_{i}$ $\left(u_{i} \in U\right)$. Thus $y \bar{z}_{i}=y\left(\bar{y} u_{i}\right)\left(\right.$ from 5.10) $=y u_{i}=z_{i}$, so that $z_{1}=y \bar{z}_{1}=y \bar{z}_{2}(5.8)=z_{2}$, whence a contradiction. Since all nonzero elements are decomposable (in accordance with the convention introduced in 10.7), $y=o$.

COROLLARY. Every nonzero element has infinite order over $U$.

(From 11.5 and 11.6.)

11.7. Theorem. There exists a disjoint sequence $\left\{a_{n}\right\}$ (finite or infinite) such that $\mathrm{V} a_{n}=e$ and each $a_{n}$ is homogeneous of infinite order over $U\left({ }^{20}\right)$.

Let $\mathfrak{m}_{1}$ be the smallest of the orders of nonzero elements $x$ over $U$; thus $\mathrm{m}_{1}$ is infinite, as just shown, and from 11.2 each $x$ of order $\mathrm{m}_{1}$ over $U$ is homogeneous. Let $a_{1}=\mathrm{V}\left\{x \mid x\right.$ is of order $\mathrm{m}_{1}$ over $\left.U\right\}$. The countable chain condition shows that $a_{1}$ is the supremum of a countable set of such elements $x$; and 11.4 then shows that $a_{1}$ is homogeneous of order $m_{1}$ over $U$. The construction is iterated transfinitely, $a_{\alpha}$ being defined as the supremum of all nonzero elements less than or equal to $-\bigvee_{\beta<\alpha} a_{\beta}$ (if any) having the smallest possible order $\mathfrak{m}_{\alpha}$ over $U$; as before, $a_{\alpha}$ is homogeneous of order $\mathfrak{m}_{\alpha}$ over $U$. The construction terminates for some $\alpha_{0}<\omega_{1}$, for the elements $a_{\alpha}$ are disjoint and nonzero, and we have $e=\mathrm{V}_{\alpha<\alpha_{0}} a_{\alpha}$. All that remains is to reorder the elements $a_{\alpha}\left(\alpha<\alpha_{0}\right)$ into a simple sequence.

12. Scalar multiplication of equivalence classes.

12.1 We could now consider the relative abstract measure algebras $E\left(a_{n}\right)$, in which all nonzero elements are now decomposable, bounded, and (from 11.3 and 8.3) homogeneous over the algebra of relatively invariant elements. However, though it would be possible to prove a representation theorem for each $E\left(a_{n}\right)$, it would not be easy to extend the representation to all of $E$; this is because (roughly speaking) the elements $a_{n}$ are not related in any convenient way to their invariant closures in $E$. In the next section we shall replace them by elements which are "comparable," in the desired way, with their invariant closures in $E$. Here we shall define and develop the necessary properties of this comparability. It would be possible to elaborate these notions further into a systematic arithmetic of equivalence classes (cf. footnote 17 ), but we shall confine ourselves to the properties actually needed in the sequel.

12.2. Given $x \neq 0$, and a positive integer $n$, there exist $n$ disjoint equiva-

(20) This theorem generalizes the decomposition of a numerical measure algebra into homogeneous parts; cf. [11]. 
lent nonzero elements $y_{i} \leqq x(i=1, \cdots, n)$.

For $x$ is decomposable (cf. 10.7), so there exist $z_{1}, z_{2} \leqq x$ such that $z_{i} \neq 0$, $z_{1} z_{2}=0$, and $z_{1} \sim z_{2}$. Since $z_{1}$ is decomposable, there exist $z_{11}, z_{12} \leqq z_{1}$ such that $z_{1 i} \neq 0, z_{11} z_{12}=0$, and $z_{11} \sim z_{12}$; and from 6.2 there exist disjoint elements $z_{21}$, $z_{22} \leqq z_{2}$ such that $z_{21} \sim z_{22} \sim z_{1 i}$. Proceeding in this way we obtain, for each $m$, $2^{m}$ disjoint equivalent nonzero elements less than or equal to $x$; and the result follows.

12.3. In 12.2 we can further suppose $\mathrm{V}_{i}=x$.

This follows from 12.2 by the usual "exhaustion" argument.

12.4. If $y_{1}, y_{2}, \cdots, y_{n}$ are disjoint equivalent elements, and if $z_{1}, z_{2}, \cdots$, $z_{n}$ are disjoint equivalent elements such that $\mathrm{V} y_{i} \sim \mathrm{V}_{i}$, then $\boldsymbol{y}_{i} \sim z_{i}$.

Let $p_{i}, q_{i}$ be maximal equivalent sub-elements of $y_{i}, z_{i}(7.5)$. Since all elements are now bounded (10.7), it readily follows from 7.5(ii) that the $2 n$ elements $p_{i}, q_{i}$, are all equivalent. Write $h_{i}=y_{i}-p_{i}, k_{i}=z_{i}-q_{i}$; thus $\bar{h}_{i} \bar{k}_{i}=o$, and (6.1) we have $h_{1} \sim h_{2} \sim \cdots \sim h_{n}, k_{1} \sim k_{2} \sim \cdots \sim k_{n}$, and $\mathrm{V} h_{i} \sim \vee k_{i}$. Thus (from 5.6 and 5.8) $\mathrm{Cl}\left(h_{i}\right)=\mathrm{Cl}\left(\mathrm{V} h_{i}\right)=\mathrm{Cl}\left(\mathrm{V}_{i}\right)=\mathrm{Cl}\left(k_{i}\right)$; and the relation $\bar{h}_{i} \bar{k}_{i}=o$ now gives $\bar{h}_{i}=o=\bar{k}_{i}$. Thus $y_{i}=p_{i} \sim q_{i}=z_{i}$.

12.5. Definitions. Given any $x$ and any positive integer $n, 12.3$ shows that, for each $x^{\prime} \in[x]$ (that is, $x^{\prime} \sim x$ ) we can write $x^{\prime}=\bigvee_{1}^{n} y_{i}$ where the $n$ elements $y_{i}$ are disjoint and equivalent; and 12.4 then shows that the equivalence class $\left[y_{1}\right]\left(=\left[y_{i}\right]\right)$ is uniquely determined. We write $\left[y_{1}\right]=(1 / n)[x]$, and more generally $\left[y_{1} \bigvee y_{2} \bigvee \cdots \vee y_{m}\right]=(m / n)[x]$, for each $m \leqq n$. Thus $(m / n) x$ is uniquely determined by the equivalence class $x$ and the integers $m, n(0 \leqq m \leqq n \geqq 1)$. Clearly $(0 / n)[x]=o,(n / n)[x]=[x],(m / n)[o]=[o]$.

The following properties follow easily from this definition, together with the results in $\$ 7$.

(1) If $p \geqq 1, n \geqq 1$, and $0 \leqq m \leqq n$, then $(m p / n p)[x]=(m / n)[x]$.

Thus $(m / n)[x]$ depends only on $[x]$ and the value of the proper fraction $m / n$. If $m / n=\rho$, we write $(m / n)[x]$ as $\rho[x]$. In the following statements, $\rho$, $\rho_{1}$, and so on denote non-negative rational numbers not exceeding 1 .

(2) If $\rho_{1}<\rho_{2}$, then $\rho_{1}[x] \leqq \rho_{2}[x]$, with equality only if $[x]=o$.

(Immediate on writing $\rho_{1}$ and $\rho_{2}$ with a common denominator.)

(3) If $[x]<[y]$, then $\rho[x] \leqq \rho[y]$, with equality only if $\rho=0$.

(4) If $[y]=\rho_{1}[x]$ and $[z]=\rho_{2}[x]$, where $y \vee z \leqq x, x \neq o$, and $y z=o$, then $\rho_{1}+\rho_{2} \leqq 1$ and $[y \vee z]=\left(\rho_{1}+\rho_{2}\right)[x]$.

(5) If $\left[y_{n}\right]=\rho\left[x_{n}\right]$, where the elements $y_{n}$ are disjoint, and the elements $x_{n}$ are disjoint, then $\left[\mathrm{V} y_{n}\right]=\rho\left[\mathrm{V} x_{n}\right]$.

(6) If $[y]=\rho_{1}[x]$, then $\rho_{2}[y]=\left(\rho_{1} \rho_{2}\right)[x]$.

The next step will consist in extending this definition of "multiplication" of equivalence classes by (rational) scalars, to allow the multipliers to be irrational. Several lemmas are needed.

12.6. If $[x] \leqq \rho[e]$ for arbitrarily small values of $\rho$, then $x=o$.

For, from (2) above, we have $[x] \leqq\left(1 / 2^{n}\right)[e]$ for every $n$. Now (12.3) we 
can write $e=f_{1} \bigvee g_{1}$ where $f_{1}, g_{1}$ are disjoint and equivalent. Similarly, $g_{1}=f_{2}$ $\vee g_{2}$ where $f_{2}, g_{2}$ are disjoint and equivalent; and so on. The disjoint sequence $\left\{f_{n}\right\}$ is such that $\left[f_{n}\right]=\left(1 / 2^{n}\right)[e]$; hence there exists $x_{n} \leqq f_{n}$ such that $x_{n} \sim x$. Then $\bigvee_{1}^{\infty} x_{n} \sim \bigvee_{2}^{\infty} x_{n}$; but all elements are now bounded (10.7), and so $x_{1}=0$, whence $x=0$.

12.7. If $\left[y_{n}\right] \leqq\left(1 / 2^{n}\right)[x](n=1,2, \cdots)$ then $\left[\mathrm{V} y_{n}\right] \leqq[x]$.

This is proved by essentially the same argument as in 12.6 , since there is no loss in assuming the elements $y_{n}$ to be disjoint.

12.8. Suppose $x$ and $y$ are such that, for each positive integer $n$, there exist $p_{n}, q_{n} \in E$ such that $\left[p_{n}\right] \leqq\left(1 / 2^{n+1}\right)[e], q_{n} \leqq\left(1 / 2^{n+1}\right)[e]$, and $\left[x-p_{n}\right]$ $\leqq\left[y \vee q_{n}\right]$. Then $[x] \leqq[y]$.

There is no loss in assuming $p_{n} \leqq x$. Then there exist $x_{n} \sim x$ and $r_{n} \sim p_{n}$ such that $r_{n} \leqq x_{n}$ and $x_{n}-r_{n} \leqq y \vee q_{n}$. Thus $x_{n} \leqq y \vee r_{n} \vee q_{n} \leqq y \vee f_{n}$, where $f_{n}=\bigvee\left\{r_{m} \vee q_{m} \mid m \geqq n\right\}$. Now $\left[r_{n} \vee q_{n}\right] \leqq\left(1 / 2^{n}\right)[e]$, from 12.5(4); hence $\left[f_{n}\right] \leqq\left(1 / 2^{n-1}\right)[e]$, as readily follows from 12.7 . Thus (12.6) $\Lambda f_{n}=o$. But (7.2) we have $[x] \leqq\left[\Lambda\left(y \vee f_{n}\right)\right]=\left[y \vee \Lambda f_{n}\right]=[y]$.

12.9. Given $x \in E$ and a real number $\sigma$ such that $0 \leqq \sigma \leqq 1$, there exists $y \in E$ having the following property: Given any positive rational number $\epsilon<1$, there exist (i) a rational number $\rho(0 \leqq \rho \leqq 1)$ such that $|\rho-\sigma| \leqq \epsilon$, (ii) an element $d \in E$ such that $[d] \leqq \epsilon[e]$ and $\left[y+{ }_{2} d\right]=\rho[x]$.

As in the proof of 12.6 , we construct a disjoint sequence of elements $f_{n} \leqq x$ such that, on writing $g_{n}=x-\bigvee\left\{f_{m} \mid m \leqq n\right\}$, we have $\left[f_{n}\right]=\left[g_{n}\right]$ $=\left(1 / 2^{n}\right)[x]$. Expand $\sigma$ as a binary decimal, say $\sigma=\sum_{1}^{\infty} m_{n} / 2^{n}$, where each $m_{n}$ is 0 or 1 , and define $t_{n}=f_{n}$ if $m_{n}=1$, and $t_{n}=0$ otherwise. Write $y=V t_{n}$. To satisfy the requirements, we have only to choose $n$ so that $2^{-n}<\epsilon$, and take $d=\mathrm{V}\left\{t_{m} \mid m>n\right\}$ and $\rho=\sum_{1}^{n} m_{k} / 2^{k}$. Clearly $|\rho-\sigma|<2^{-n}$ and (from $12.5(4))\left[y+{ }_{2} d\right]=\rho[x]$. Finally, the fact that $[d] \leqq\left(1 / 2^{n}\right)[e]$ follows from 12.7 .

REMARK. The proof shows that a little more is true than was stated; we can further make $d \leqq y, \rho \leqq \sigma$, and $[d] \leqq \epsilon[x]$.

12.10. Given $x \in E$ and $\sigma(0 \leqq \sigma \leqq 1)$, suppose that $y^{\prime}$ also has the same property as $y$ in 12.9 ; then $y^{\prime} \sim y$.

For each $n$ we can write

$$
\begin{aligned}
& {\left[y+{ }_{2} d_{n}\right]=\rho_{n}[x], \quad\left|\rho_{n}-\sigma\right|<1 / 2^{n+2}, \quad\left[d_{n}\right] \leqq\left(1 / 2^{n+2}\right)[e],} \\
& {\left[y^{\prime}+{ }_{2} d_{n}^{\prime}\right]=\rho_{n}^{\prime}[x], \quad\left|\rho_{n}^{\prime}-\sigma\right|<1 / 2^{n+2}, \quad\left[d_{n}^{\prime}\right] \leqq\left(1 / 2^{n+2}\right)[e],}
\end{aligned}
$$

and can suppose without loss that $\rho_{n}^{\prime} \geqq \rho_{n}$ for infinitely many values of $n$. Thus we find $d_{n}^{\prime \prime} \leqq y^{\prime}+{ }_{2} d_{n}^{\prime}$ such that $\left[d_{n}^{\prime \prime}\right]=\left(\rho_{n}^{\prime}-\rho_{n}\right)[x] \leqq\left(1 / 2^{n+1}\right)[e]$, and then have (6.1) $\left[\left(y^{\prime}+{ }_{2} d_{n}^{\prime}\right)-d_{n}^{\prime \prime}\right]=\rho_{n}[x]$. Hence $\left(y^{\prime}+{ }_{2} d_{n}^{\prime}\right)-d_{n}^{\prime \prime} \sim y+{ }_{2} d_{n}$ for arbitrarily large $n$; and from 12.8 it readily follows that $[y] \leqq\left[y^{\prime}\right] \leqq[y]$. Since $y$ is bounded, we obtain $\left[y^{\prime}\right]=[y]$.

12.11. Definition. If $x, \sigma$ and $y$ are related as in 12.9 , we write $[y]=\sigma[x]$; from 12.9 and 12.10 , this relation determines one and only one equivalence 
class $[y]$ when $[x]$ and $\sigma(0 \leqq \sigma \leqq 1)$ are given. Further, if $\sigma$ is rational this definition reduces to that of 12.5 , since in 12.9 we can then take $d=o$ and $\rho=\sigma$.

Throughout the rest of this section, $\sigma, \sigma_{1}$, and so on will denote real numbers between 0 and 1 (inclusive), and $\epsilon$ denotes a real number such that $0<\epsilon<1$. Most of the proofs will be omitted, since they involve no new ideas.

12.12. If $y \leqq x$ and $[y]=\sigma[x]$, then $[x-y]=(1-\sigma)[x]$.

This is immediate from the way in which $y$ was constructed in 12.9 , if we note that $\mathrm{V}_{n}=x$ there (since $\Lambda g_{n}=o$, from 12.6).

12.13. If $[y]=\sigma[x]$, and $u$ is invariant, then $[u y]=\sigma[u x]$.

Immediate from 6.6 and the definitions -12.5 and 12.11 .

12.14. If $\sigma_{1} \leqq \sigma_{2}, \sigma_{1}[x] \leqq \sigma_{2}[x]$.

This follows from 12.8 by an argument similar to that in 12.10 .

Corollary. If $\sigma_{1}<\sigma_{2}, \sigma_{1}[x]<\sigma_{2}[x]$ unless $x=o$.

For we can choose rational $\rho_{1}, \rho_{2}$ such that $\sigma_{1}<\rho_{1}<\rho_{2}<\sigma_{2}$; the corollary now follows from 12.14 and $12.5(2)$.

12.15. If $[p]=\sigma_{1}[x],[q]=\sigma_{2}[x], p q=o, p \vee q \leqq x$, and $x \neq o$, then $\sigma_{1}+\sigma_{2} \leqq 1$ and $[p \vee q]=\left(\sigma_{1}+\sigma_{2}\right)[x]$

That $\sigma_{2} \leqq 1-\sigma_{1}$ follows easily from 12.12 . The remainder of the assertion follows from the definition of $\left(\sigma_{1}+\sigma_{2}\right)[x]$ and from the rational case $(12.5(4))$.

12.16. If $\left\{y_{n}\right\}$ is a disjoint sequence such that $y_{n} \leqq x \neq 0$ and $\left[y_{n}\right]=\sigma_{n}[x]$, then $\sigma \equiv \sum \sigma_{n} \leqq 1$, and $\left[\mathrm{V} y_{n}\right]=\sigma[x]$.

An easy induction based on 12.15 gives $\sum_{1}^{m} \sigma_{n} \leqq 1$, for each $m$, so that $\sigma \leqq 1$. It also gives $\left[\mathrm{V}_{1}^{m} y_{n}\right]=\left(\sum_{1}^{m} \sigma_{n}\right)[x] \leqq \sigma[x](12.14)$, for each $m$; hence (7.3) $\left[\mathrm{V}_{y_{i}}\right] \leqq \sigma[x]$. On the other hand, we can assume $\sigma>0$; let $\epsilon$ be any positive number less than $\sigma$. If $N$ is large enough, we have (on using 12.15 and 12.14) $\left[\mathrm{V} y_{n}\right] \geqq\left[\mathrm{V}_{1}^{N} y_{n}\right]=\left(\sum_{1}^{N} \sigma_{n}\right)[x] \geqq(\sigma-\epsilon)[x]$; and it readily follows from 12.8 that $\left[\mathrm{V} y_{n}\right] \geqq \sigma[x]$. Thus, since all elements are now bounded, $\left[\mathrm{V} y_{n}\right]$ $=\sigma[x]$.

12.17. If $\left\{y_{n}\right\}$ and $\left\{z_{n}\right\}$ are two sequences, each disjoint, such that $\left[y_{n}\right]=\sigma\left[z_{n}\right](n=1,2, \cdots)$, then $\left[\mathrm{V} y_{n}\right]=\sigma\left[\mathrm{V} z_{n}\right]$.

We may suppose $y_{n} \leqq z_{n}$. Given any rational $\epsilon>0$ (and $<1$ ) we choose a rational number $\rho$ so that $\sigma-\epsilon<\rho<\sigma$, and then (12.9, Remark) choose $d_{n} \leqq y_{n}$ so that $\left[d_{n}\right] \leqq\left(\epsilon / 2^{n}\right)[e]$ and $\left[y_{n}-d_{n}\right]=\rho_{n}\left[z_{n}\right]$, where $\rho<\rho_{n}<\sigma$ and $\rho_{n}$ is rational. The rest is routine.

12.18. If $x \geqq y \geqq z$, and $x \neq o$, and if $[y]=\sigma_{1}[x]$ and $z=\sigma_{2}[x]$, then $\sigma_{1} \geqq \sigma_{2}$ and $[y-z]=\left(\sigma_{1}-\sigma_{2}\right)[x]$.

12.19. If $y_{1} \leqq y_{2} \leqq \cdots \leqq x$, and if $\left[y_{n}\right]=\sigma_{n}[x]$ and $x \neq 0$, then $\sigma_{1} \leqq \sigma_{2}$ $\leqq \cdots \leqq 1$, and $\left[\mathrm{V} y_{n}\right]=\left(\lim _{n \rightarrow \infty} \sigma_{n}\right)[x]$.

(From 12.16 and 12.18 applied to the relation $\left.\mathrm{V}_{n}=\mathrm{V}\left(y_{n}-y_{n-1}\right).\right)$

A similar result holds for decreasing sequences.

12.20. If $[y]=\sigma_{1}[x]$, then $\sigma_{2}[y]=\left(\sigma_{1} \sigma_{2}\right)[x]$.

This will be needed only in the case in which $\sigma_{1}$ is rational, when it fol- 
lows fairly easily from $12.5(6)$ and the definition (12.11). The extension to the general case can then be derived, though with more trouble.

\section{Decomposition into parts comparable with their closures.}

13.1. We begin with a fundamental result which, roughly speaking, asserts that the abstract measures can be approximated by "step functions."

Theorem. Given $x \in E$ and a positive integer $n$, there exist $n$ disjoint invariant elements $u_{1}, u_{2}, \cdots, u_{n}$ such that (i) $\bigvee u_{i}=e$, and (ii) $((i-1) / n)\left[u_{i}\right]$ $\leqq\left[x u_{i}\right] \leqq(i / n)\left[u_{i}\right](1 \leqq i \leqq n)$.

By 12.3, we can write $e=t_{1} \bigvee t_{2} \bigvee \ldots \vee t_{n}$, where the elements $t_{i}$ are disjoint and equivalent. For each $i$, consider the elements $x$ and $t_{1} \bigvee t_{2} \bigvee \cdots \vee t_{i}$; from 7.6 there exists $w_{i} \in U$ such that

(a) $\left[x w_{i}\right] \leqq\left[w_{i}\left(t_{1} \vee \cdots \vee t_{i}\right)\right]$, (b) if $v \in U\left(-w_{i}\right)$ then $[x v] \geqq\left[v\left(t_{1} \vee \cdots\right.\right.$ $\left.\left.\bigvee t_{i}\right)\right]$, with equality only if $v=o$. Now $\left[t_{1} \bigvee \cdots \vee t_{i}\right]=(i / n)[e]$, by definition; hence, from 12.13, properties (a) and (b) may be restated as:

$\left(\mathrm{a}^{\prime}\right)\left[x w_{i}\right] \leqq(i / n)\left[w_{i}\right],\left(\mathrm{b}^{\prime}\right)$ if $v \in U\left(-w_{i}\right)$ then $[x v]>(i / n)[v]$ unless $v=o$.

We assert: $o \leqq w_{1} \leqq w_{2} \leqq \cdots \leqq w_{n}=e$. For suppose $i<j$ and let $v=w_{i}-w_{j}$; if $v \neq o$, we have at once from 12.13 that $[x v]>(j / n)[v]>(i / n)[v](12.5(2))$ $\geqq[x v]$, a contradiction. Thus $v=o$ and $w_{i} \leqq w_{j}$. The fact that $w_{n}=e$ is immediate from $\left(\mathrm{b}^{\prime}\right)$.

Write $w_{0}=o$, and define $u_{i}=w_{i}-w_{i-1}(1 \leqq i \leqq n)$. Thus the elements $u_{1}, \cdots, u_{n}$ are invariant and disjoint, and $\mathrm{V} u_{i}=e$. From $\left(\mathrm{a}^{\prime}\right),\left(\mathrm{b}^{\prime}\right)$ and 12.13 we have $((i-1) / n)\left[u_{i}\right] \leqq\left[x u_{i}\right] \leqq(i / n)\left[u_{i}\right]$ as desired; and have in fact more 一that $((i-1) / n)\left[u_{i}\right]<\left[x u_{i}\right]$ if $i<n$, unless $u_{i}=o$.

13.2 LemmA. Given $x \neq o$, there exists $y \in E$ such that $o<y \leqq x$ and $[y]$ $=\rho[\bar{y}]$ for some rational $\rho(0<\rho \leqq 1)$.

We apply 13.1, and note that if $n$ is large enough $u_{1} \neq e$, since otherwise $[x] \leqq(1 / n)[e]$ for arbitrarily large $n$, which is impossible (12.6). Thus for some $i>1$ we have $u_{i} \neq 0$; and since $((i-1) / n)\left[u_{i}\right] \leqq\left[x u_{i}\right]$ there exists $y \leqq x u_{i}$ such that $[y]=((i-1) / n)\left[u_{i}\right]$. Since $i>1$ and $u_{i} \neq o$ we have $y \neq o$; and, from 12.13, $[\bar{y} y]=\rho\left[\bar{y} u_{i}\right]$ (where $\left.\rho=(i-1) / n\right)$, that is, $[y]=\rho[\bar{y}]$.

13.3. Theorem. Given $x \neq o$, there exists a sequence $\left\{z_{n}\right\}$ (finite or infinite) of disjoint nonzero elements such that (i) $\mathrm{V} z_{n}=x$, (ii) $\left[z_{n}\right]=\rho_{n}\left[\bar{z}_{n}\right]$, where $0<\rho_{n} \leqq 1$ and $\rho_{n}$ is rational.

Let $\left\{z_{n}\right\}$ be a maximal disjoint collection of nonzero elements $\leqq x$ satisfying (ii). From 13.2 it readily follows that $\mathrm{V}_{n}=x$, proving the theorem.

REMARK. The theorem also holds if $x=o$ provided we adopt suitable conventions about "empty" sequences.

13.4. Theorem. There exists a disjoint sequence $\left\{e_{n}\right\}$ (finite or infinite) such that (i) $V e_{n}=e$, (ii) each $e_{n}$ is homogeneous of infinite order $\mathfrak{m}_{n}$ over $U$, (iii) for each $n,\left[e_{n}\right]=\rho_{n}\left[\bar{e}_{n}\right]$, where $\rho_{n}$ is rational and $0<\rho_{n} \leqq 1$. 
From 11.7 there exist disjoint (nonzero) elements $a_{n}$ such that $\mathrm{V} a_{n}=e$ and $a_{n}$ is homogeneous of infinite order over $U$. Applying 13.3, we write $a_{n}=\mathrm{V}_{m} e_{n m}$ where (for each $n$ ) the elements $e_{n m}$ are disjoint, nonzero, and $\left[e_{n m}\right]=\rho_{n m}\left[\bar{e}_{n m}\right]$. From the definition of homogeneity (11.1), each $e_{n m}$ is homogeneous of infinite order over $U$. We have only to renumber the elements $e_{n m}$ into a single sequence $\left\{e_{n}\right\}$.

This theorem provides the desired decomposition of $e\left(=e^{\prime}\right.$ or $f^{n}$; cf. $10.7)$. In the next sections $(\$ \$ 14-17)$ we shall consider the relative algebras $\left\{E\left(e_{n}\right), \sim\right\}$, showing that each is isomorphic with the direct product of a numerical measure algebra with a trivial abstract measure algebra. It will then be relatively easy $(\S \S 18,19)$ to derive a product representation for all of $E$.

\section{Structure of $E\left(e_{n}\right)$; separable case.}

14.1. Let $e_{n}$ be one of the elements in 13.4, and suppose that its order over $U$ is exactly $\boldsymbol{\aleph}_{0}$. In this section we shall analyze the structure of the relative algebra $E\left(e_{n}\right)$; and, as all considerations here will be relative to $e_{n}$, we shall write $E\left(e_{n}\right)$ simply as $E, e_{n}$ as $e$, and the relative sub-algebra of relatively invariant elements, $U_{e_{n}}$, as $U$. From 11.3 and 8.3 , we can thus assume that every nonzero $x \in E$ has order $\aleph_{0}$ over $U$, and is also decomposable and bounded (10.7). We shall deduce the following theorem:

THEOREM. There exists a sub-algebra $P$ of $E$ such that

(i) $E=\mathbb{B}(P \cup U)$,

(ii) $(P, \sim)$ is naturally isomorphic to a numerical measure algebra $(P, \mu)$, in such a way that, for each $p \in P,[p]=\mu(p)[e]$,

(iii) $(P, \mu)$ is isometric to $I^{1}$ (cf. 3.1(a)).

14.2. Since the order of $e$ over $U$ is now $\boldsymbol{\aleph}_{0}$, there exists a countably infinite set $B=\left\{b_{n}\right\}(n=1,2, \cdots)$ such that $E=\mathbb{B}(B \cup U)$. The first step in the proof consists in replacing the elements $b_{n}$ by others with improved properties; and this is done in two stages.

14.3. The elements $c(\tau)$. Let $\tau$ denote any fraction of the form $i / 4^{n}(n \geqq 0$, $\left.0 \leqq i \leqq 4^{n}\right)$. We define $c(\tau)$ by induction, as follows: When $n=0$, we set $c(0)=o$ and $c(1)=e$. When $c\left(i / 4^{n}\right)$ has been defined (for a particular $n \geqq 0$ and for all $i$ such that $\left.0 \leqq i \leqq 4^{n}\right)$, we choose elements $f\left(i / 4^{n}\right), g\left(i / 4^{n}\right)$, such that $f\left(i / 4^{n}\right) \leqq b_{n+1}\left\{c\left(i / 4^{n}\right)-c\left((i-1) / 4^{n}\right)\right\}=h\left(i / 4^{n}\right)$, say, $g\left(i / 4^{n}\right) \leqq\left(-b_{n+1}\right)\left\{c\left(i / 4^{n}\right)\right.$ $\left.-c\left((i-1) / 4^{n}\right)\right\}=k\left(i / 4^{n}\right)$, and $\left[f\left(i / 4^{n}\right)\right]=(1 / 2)\left[h\left(i / 4^{n}\right)\right],\left[g\left(i / 4^{n}\right)\right]=(1 / 2)$ $\left[k\left(i / 4^{n}\right)\right]$, using 12.3 , and then define (for $1 \leqq i \leqq 4^{n}$ )

and

$$
\begin{aligned}
& c\left((4 i-4) / 4^{n+1}\right)=c\left((i-1) / 4^{n}\right), \\
& c\left((4 i-3) / 4^{n+1}\right)=c\left((i-1) / 4^{n}\right) \vee f\left(i / 4^{n}\right), \\
& c\left((4 i-2) / 4^{n+1}\right)=c\left((i-1) / 4^{n}\right) \bigvee h\left(i / 4^{n}\right),
\end{aligned}
$$

$$
c\left((4 i-1) / 4^{n+1}\right)=c\left((4 i-2) / 4^{n+1}\right) \vee g\left(i / 4^{n}\right) \text {. }
$$


It readily follows by induction that

(1) If $\tau_{1} \leqq \tau_{2}$ then $c\left(\tau_{1}\right) \leqq c\left(\tau_{2}\right)$, and that

(2) $\left[c\left(i / 4^{n}\right)-c\left((i-1) / 4^{n}\right] \leqq\left(1 / 2^{n}\right)[e]\right.$.

Again, we have

$$
\begin{aligned}
\bigvee_{i=1}^{4^{n}}\left\{c\left((4 i-2) / 4^{n+1}\right)-c\left((4 i-4) / 4^{n+1}\right)\right\} & =\bigvee h\left(i / 4^{n}\right) \\
& =b_{n+1} \bigvee\left\{c\left(i / 4^{n}\right)-c\left((i-1) / 4^{n}\right)\right\} \\
& =b_{n+1}
\end{aligned}
$$

hence, on writing $C=$ set of all elements $c(\tau)$, we have $B \subset \mathcal{B}(C)$, and therefore

$$
E=\mathbb{B}(C \cup U) \text {. }
$$

14.4. The elements $d_{\rho}$. Now let $\rho$ be any rational number such that $0 \leqq \rho \leqq 1$. Choose any $y_{\rho}$ such that $\left[y_{\rho}\right]=\rho[e]$; an application of 7.6 to the elements $c(\tau), y_{\rho}$, then gives an invariant element $u_{\tau \rho}$ such that (on using 12.13)

(4) $[c(\tau) u] \leqq \rho[u]$ whenever $u \in U\left(u_{\tau \rho}\right)$, and $[c(\tau) v]>\rho[v]$ whenever $v \in U\left(-u_{\tau \rho}\right)$, unless $v=0$.

It readily follows (by the same argument as in 13.1) that

(5) $u_{\tau_{1 \rho} \rho} \geqq u_{\tau_{2 \rho}}$ if $\tau_{1} \leqq \tau_{2} ; u_{0 \rho}=e ; u_{1 \rho}=o$ unless $\rho=1$; and $u_{11}=e$.

Similarly

(6) $u_{\tau \rho_{1}} \leqq u_{\tau \rho_{2}}$ if $\rho_{1} \leqq \rho_{2} ; c(\tau) u_{\tau 0}=o$; and $u_{\tau 1}=e$.

Now define, for each $n$,

$$
d_{\rho n}=\bigvee\left\{c(\tau) u_{\tau \rho} \mid \tau=i / 4^{n}, 0 \leqq i \leqq 4^{n}\right\},
$$

and define

$$
d_{\rho}=\mathrm{V}\left\{c(\tau) u_{\tau \rho}, \text { all } \tau\right\} .
$$

It readily follows that $d_{\rho 1} \leqq d_{\rho 2} \leqq \cdots$, and $V_{n} d_{\rho n}=d_{\rho}$. Further, from (6) we have

$$
d_{\rho_{1}} \leqq d_{\rho_{2}} \quad \text { if } \quad \rho_{1} \leqq \rho_{2} ; \text { and } d_{0}=o, d_{1}=e .
$$

We next show that

$$
\left[d_{\rho}\right]=\rho[e] .
$$

Since $c\left((i+1) / 4^{n}\right) \geqq c\left(i / 4^{n}\right)$, we have (on writing $u_{\tau p}=0$ if $\tau>1$, so that (5) still holds)

$$
d_{\rho n}=\bigvee_{i=0}^{4^{n}} c\left(i / 4^{n}\right) v_{\rho n i}, \quad \text { where } \quad v_{\rho n i}=u_{\left(i / 4^{n}\right) \rho}-u_{\left((i+1) / 4^{n}\right) \rho} .
$$

From (4) and 12.5(5) it follows that $\left[d_{\rho n}\right] \leqq \rho\left[\mathrm{V}_{i} v_{\rho n i}\right]=\rho[e]$; and therefore (7.3) we have $\left[d_{p}\right] \leqq \rho[e]$. 
To obtain the opposite inequality, we note that (from (7)) we may assume $\rho<1$, so that now $u_{1 \rho}=0$; thus $v_{\rho n 4^{n}}=0$, so that

$$
d_{\rho n} \leqq \bigvee_{i=0}^{4^{n}-1} c\left((i+1) / 4^{n}\right) v_{\rho n i}=q_{\rho n}, \quad \text { say. }
$$

But $q_{\rho n}-d_{\rho n}=\bigvee_{i=0}^{4^{n}-1}\left[c\left((i+1) / 4^{n}\right)-c\left(i / 4^{n}\right)\right\} v_{\rho n i}$; and from (2) and 12.13 it now follows that $\left[q_{\rho n}-d_{\rho n}\right] \leqq\left(1 / 2^{n}\right)\left[\mathrm{V}_{i} v_{\rho n i}\right]=\left(1 / 2^{n}\right)[e]$. A fortiori, therefore, $\left[q_{\rho n}-d_{\rho}\right] \leqq\left(1 / 2^{n}\right)[e]$. But from (4) we have $\left[c\left((i+1) / 4^{n}\right) v_{\rho n i}\right] \geqq \rho\left[v_{\rho n i}\right]$, whence $\left[q_{\rho n}\right] \geqq \rho[e]$. It readily follows from 12.8 that $\left[d_{\rho}\right] \geqq \rho[e]$; and thus $\left[d_{\rho}\right]=\rho[e]$, Q.E.D.

Let $D$ denote the set of all elements $d_{\rho}$. We shall now prove that $C \subset B(D \cup U)$. For convenience, we define $d_{\rho}=o$ for $\rho<0$, and $d_{\rho}=e$ for $\rho>1$; thus (7) is still maintained.

By 13.1 there exist, for each $\tau\left(=j / 4^{m}\right)$ and each $n>0, n$ disjoint invariant elements $w_{\tau n i}(1 \leqq i \leqq n)$ such that

$$
\bigvee_{i} w_{\tau n i}=e,
$$

and

$$
((i-1) / n)\left[w_{\tau n i}\right] \leqq\left[c(\tau) w_{\tau n i}\right] \leqq(i / n)\left[w_{\tau n i}\right] \quad(1 \leqq i \leqq n) .
$$

From 12.13 and (4) we see that, on writing $\rho=(i-2) / n$ (where it is assumed for the moment that $i \geqq 2$ ), we have $w_{\tau n i} u_{\tau \rho}=o$, and therefore (from (5)) $w_{\tau n i} u_{\tau^{\prime} \rho}=o$ if $\tau^{\prime} \geqq \tau$. Hence

$$
d_{\rho} w_{\tau n i}=\underset{\tau^{\prime}}{\bigvee} c\left(\tau^{\prime}\right) w_{\tau n i} u_{\tau^{\prime} \rho} \leqq \underset{\tau^{\prime} \leqq \tau}{\bigvee} c\left(\tau^{\prime}\right) \leqq c(\tau)
$$

so that

$$
d_{\rho} w_{\tau n i} \leqq c(\tau) w_{\tau n i}, \text { where } \rho=(i-2) / n,
$$

a result which holds for all $i \geqq 0$ since $d_{\rho}=o$ if $\rho<0$. It follows that

$$
c(\tau) \geqq \bigvee_{i, n} d_{\rho} w_{\tau n i} \quad(\rho=(i-2) / n, 1 \leqq i \leqq n) .
$$

To obtain the reverse inequality, let $c^{\prime}(\tau)$ denote the element on the right of (12). Since $\left[c(\tau) w_{\tau n i}\right] \leqq(i / n)\left[w_{\tau n i}\right]$ (from (10)), while if $i \geqq 2 \quad\left[d_{\rho} w_{t n i}\right]$ $=((i-2) / n)\left[w_{\tau n i}\right]$ (from (8) and 12.13), we readily obtain $\left[c(\tau) w_{\tau n i}-d_{\rho} w_{\tau n i}\right]$ $\leqq(2 / n)\left[w_{\tau n i}\right]$, a result which also holds (trivially) for $i=0,1$. Hence, on summing over $i$, we obtain (using (9) and 12.17)

$$
(2 / n)[e] \geqq\left[c(\tau)-\bigvee_{i} d_{\rho} w_{\tau n i}\right] \geqq\left[c(\tau)-c^{\prime}(\tau)\right]
$$

and from 12.6 it follows that $c(\tau)-c^{\prime}(\tau)=o$. Thus $c(\tau)=c^{\prime}(\tau) \in \mathbb{B}(D \cup U)$; and (3) now gives 


$$
E=\mathbb{B}(D \cup U)=\mathscr{B}(P \cup U) \text {, where } P=\mathbb{B}(D) .
$$

14.5. The finitely additive algebra generated by $D$. Consider the set $F$ of all elements $x$ expressible as

$$
x=\left(d_{\rho_{1}}-d_{\rho_{2}}\right) \vee\left(d_{\rho_{3}}-d_{\rho_{4}}\right) \vee \cdots \vee\left(d_{\rho_{2 n-1}}-d_{\rho_{2 n}}\right),
$$

where $1 \geqq \rho_{1} \geqq \rho_{2} \geqq \cdots \geqq \rho_{2 n} \geqq 0$, and the numbers $\rho_{i}$ are rational. It is easy to see that, if $x$ and $y \in F$, then so do $x \vee y$ and $-x$; thus $F$ is a finitely additive sub-algebra of $E$. Clearly $\mathbb{B}(D) \supset F \supset D$ (for $d_{0}=o-(7)$ ).

From (8), 12.18 and 12.16, we obtain

(14) If $x \in F,[x]=\rho[e]$, where $\rho=\sum_{1}^{n}\left(\rho_{2 i-1}-\rho_{2 i}\right)$ (the numbers $\rho_{i}$ arising from any expression of $x$ as above, so that $\rho$ is rational and $0 \leqq \rho \leqq 1)$. Note that (from 12.14) the number $\rho$ in (14) is determined uniquely by $x$-unless $e=o$, which we shall assume not to be the case.

14.6. The $\sigma$-algebra $P^{\prime}$. Now consider the set $P^{\prime}$ of elements $y$ which have the following property: For each $\epsilon(>0$ and $<1)$ there exists $z \in E$ such that $y+{ }_{2} z \in F$ and $[z]<\epsilon[e]$.

It is easy to verify that, if $y$ and $y_{i}(1 \leqq i \leqq n)$ belong to $P^{\prime}$, then so do $-y$ and $\mathrm{V} y_{i}$; thus $P^{\prime}$ is at least a finitely additive sub-algebra of $E$. Now, we have

(15) If $y \in P^{\prime}$, there exists $\sigma(0 \leqq \sigma \leqq 1)$ such that $[y]=\sigma[e]$.

For there exist elements $z_{n}$ such that $y+{ }_{2} z_{n} \in F$ and $\left[z_{n}\right] \leqq \epsilon_{n}[e]$, where $\epsilon_{n}=1 /(n+1)$. Thus, writing $x_{n}=y+{ }_{2} z_{n}$, we have (14) $\left[x_{n}\right]=\rho_{n}[e]$, where $\rho_{n}$ is rational. Further, since $x_{m} x_{n} \in F$, we have $\left[x_{m} x_{n}\right]=\rho_{m n}[e]$, say. Thus $\left[x_{n}-x_{m} x_{n}\right]=\left(\rho_{n}-\rho_{m n}\right)[e], \quad\left[x_{m}-x_{m} x_{n}\right]=\left(\rho_{m}-\rho_{m n}\right)[e], \quad$ and so $\left[x_{m}+{ }_{2} x_{n}\right]$ $=\left(\rho_{m}+\rho_{n}-2 \rho_{m n}\right)[e]$. But $\left[x_{m}+{ }_{2} x_{n}\right]=\left[z_{m}+{ }_{2} z_{n}\right] \leqq\left[z_{m} \vee z_{n}\right] \leqq\left(\epsilon_{m}+\epsilon_{n}\right)[e]$, and so $\left(\rho_{n}-\rho_{m n}\right)+\left(\rho_{m}-\rho_{m n}\right) \leqq \epsilon_{m}+\epsilon_{n}(12.5(2))$. Thus, since each term on the left is non-negative, we have $\rho_{m n} \leqq \rho_{n} \leqq \rho_{m n}+\epsilon_{m}+\epsilon_{n}$ and $\rho_{m n} \leqq \rho_{m} \leqq \rho_{m n}+\epsilon_{m}+\epsilon_{n}$, whence $\left|\rho_{m}-\rho_{n}\right| \leqq \epsilon_{m}+\epsilon_{n}$. The sequence $\left\{\rho_{n}\right\}$ thus converges to a limit $\sigma(0 \leqq \sigma \leqq 1)$ as $n \rightarrow \infty$; and the definition of $\sigma[e]$ (12.11) shows at once that $[y]=\sigma[\bar{e}]$.

To prove that $P^{\prime}$ is a $\sigma$-subalgebra of $E$, we have only to show that if $y_{n} \in P^{\prime}$ and $y_{1} \leqq y_{2} \leqq \cdots$, then $\vee y_{n}=y$, say, $\in P^{\prime}$. Now from (15) we have $\left[y_{n}\right]=\sigma_{n}[e]$ and so (from 12.19) $y=\sigma[e]$ where $\sigma=\lim \sigma_{n}$. Thus, given $\epsilon$, there exists $n$ such that $\left[y-y_{n}\right]=\left(\sigma-\sigma_{n}\right)[e] \leqq(\epsilon / 2)[e]$. Since $y_{n} \in P^{\prime}$, there exists $z$ such that $[z] \leqq(\epsilon / 2)[e]$ and $y_{n}+{ }_{2} z \in F$. Let $z^{\prime}=y+{ }_{2} y_{n}+{ }_{2} z$; then, since $y+{ }_{2} z^{\prime} \in F$ and $\left[z^{\prime}\right] \leqq \epsilon[e]$, we have $y \in P^{\prime}$.

14.7. Since $P^{\prime} \supset F$, we have $P^{\prime} \supset \mathbb{B}(D)=P$ (in fact $P^{\prime}=P$, but this is not needed); and (15) then shows that to each $y \in P$ corresponds a unique real number $\mu(y)$, such that $0 \leqq \mu(y) \leqq 1$ and $[y]=\mu(y)[e]$. Properties 12.16, 12.14, show that $\mu$ is a countably additive numerical measure on $P$, vanishing only for $o$, and such that $\mu\left(y_{1}\right)=\mu\left(y_{2}\right)$ (where $y_{1}, y_{2} \in P$ ) if and only if $y_{1} \sim y_{2}$. That is, the sub-algebra $(P, \sim)$ of $E$ is naturally isomorphic to the numerical measure algebra $(P, \mu)$. We have already shown (13) that $E=\mathscr{B}(P \cup U)$. 
Finally, it is not hard to see that $(P, \mu)$ is isometric with $I^{1}$, the algebra of Lebesgue-measurable sets modulo null sets in the unit interval; one makes the interval $(0, \rho)$ correspond to the element $d_{\rho}$, and extends this correspondence in succession to $F$ and $P$. (We omit the details.) Thus the theorem stated in 14.1 is now proved.

15. Structure of $E\left(e_{n}\right)$; inseparable case.

15.1. Now let $e_{n}$ be one of the elements in 13.4 which has uncountable order over $U$; we analyze the structure of the relative algebra $E\left(e_{n}\right)$. As in the previous section, all considerations will be relative to $e_{n}$; thus we write $E\left(e_{n}\right)$ as $E, e_{n} U$ as $U$, and so on, and can now assume that every nonzero element of $E$ has order $\mathfrak{m}>\boldsymbol{N}_{0}$ over $U$, and is also decomposable and bounded. The fundamental theorem is the same as in the separable case (14.1), except that the measure-algebra is different.

Theorem. There exists a sub-algebra $P$ of $E$ such that

(i) $E=\mathrm{B}(P \cup U)$,

(ii) $(P, \sim)$ is naturally isomorphic to a numerical measure algebra $(P, \mu)$, in such a way that, for each $p \in P,[p]=\mu(p)[e]$,

(iii) $(P, \mu)$ is isometric to $I^{\mathfrak{m}}$ (cf. $\left.3.1(\mathrm{a})\right)$.

15.2. We shall require the following lemma.

PRINCIPAL Lemma. Let $S$ be a subalgebra of E such that (a) $S \supset U$, (b) every nonzero element of $E$ is of infinite order over $S$. Then, given any $x \in E-S$, there exists a sub-algebra $Q$ of $E$ such that (i) $x \in \mathcal{B}(Q \cup S)$, (ii) $(Q, \sim)$ is naturally $i$ somorphic to a numerical measure algebra $(Q, \mu)$, in such a way that, for each $q \in Q$ and $s \in S,[q s]=\mu(q)[s]$, and (iii) $(Q, \mu)$ is isometric to $I^{1}$.

This lemma will be proved in $\$ 16$. Taking it for granted, we now deduce the theorem.

15.3. There exists a set $A \subset E$, with $|A|=\mathfrak{m}$, such that $E=\mathscr{B}(A \cup U)$. Well-order the elements of $A$ as $a_{\alpha}, 1 \leqq \alpha<\Omega$, where $\Omega$ is the first ordinal of power $\mathrm{m}$. From the lemma (applied with $S=U$ and $x=a_{1}$ ), there exists a sub-algebra $Q_{1}$ such that $a_{1} \in \mathcal{B}\left(Q_{1} \cup U\right)$ and for each $q \in Q_{1}$ and $u \in U$ we have $[q u]=\mu_{1}(q)[u]$; further $\left(Q_{1}, \mu_{1}\right)$ is isometric to $I^{1}$. Now suppose that subalgebras $Q_{\beta}$ have been defined for all $\beta<\alpha$ (where $\alpha<\Omega$ ), in such a way that:

(i) $a_{\beta} \in \mathcal{B}\left(\cup_{\gamma \leqq \beta} Q_{\gamma} \cup U\right)$;

(ii) $\left(Q_{\beta}, \sim\right)$ is naturally isomorphic to a numerical measure algebra $\left(Q_{\beta}, \mu_{\beta}\right)$ in such a way that, on defining $S_{\beta}=\mathbb{B}\left(\cup_{\gamma<\beta} Q_{\gamma} \cup U\right)$, we have $[b c]=\mu_{\beta}(b)[c]$ for all $b \in Q_{\beta}$ and $c \in S_{\beta}$; and

(iii) $Q_{\beta}$ is isometric either to $I^{1}$ or to the trivial measure algebra $L$ consisting of $o$ and $e$ only (these elements having measures 0 and 1 respectively).

Define $Q_{\alpha}$ as follows: If $a_{\alpha} \in S_{\alpha}$, take $Q_{\alpha}$ to consist of the elements $o$ and $e$ only, with $\mu_{\alpha}(o)=0$ and $\mu_{\alpha}(e)=1$. The properties of the transfinite sequence are clearly maintained. 
If $a_{\alpha} \notin S_{\alpha}$, we first note that every nonzero element $x \in E$ is of infinite order (and in fact of order $\mathfrak{m}$ ) over $S_{\alpha}$; in fact, if $E(x)=x \mathbb{B}\left(X \cup S_{\alpha}\right)$, there exists an at most countable subset $A_{\beta}$ of $Q_{\beta}$ such that $Q_{\beta}=\mathscr{B}\left(A_{\beta}\right)$ (from the fact that $Q_{\beta}$ is isomorphic to $I^{1}$ or $\left.L\right)$, and we have $E(x)=x \mathcal{B}\left(\cup_{\beta<\alpha} A_{\beta} \cup U \cup X\right)$ so that $\left|\bigcup_{\beta<\alpha} A_{\beta} \cup X\right| \geqq \mathfrak{m}$ and therefore $|X| \geqq \mathfrak{m}$. Hence the lemma (15.2) can be applied, taking $S=S_{\alpha}$ and $x=a_{\alpha}$; the sub-algebra given by the lemma is taken to be $Q_{\alpha}$.

Thus $Q_{\alpha}$ is defined for all $\alpha<\Omega$; and since $a_{\alpha} \in S_{\alpha+1}$ we readily obtain

(1) $E=\mathrm{B}\left(S_{\Omega} \cup U\right)$, where $S_{\Omega}=\mathrm{B}\left(\cup Q_{\alpha}\right)$.

It follows (by the same order argument as before) that $Q_{\alpha}$ is isometric to $I^{1}$ for $m$ values of $\alpha$.

Suppose $x \in Q_{\alpha} \cap Q_{\beta}(\alpha \neq \beta)$. Since $[x]=\mu_{\alpha}(x)[e]$ and also $[x]=\mu_{\beta}(x)[e]$, we have (12.14) $\mu_{\alpha}(x)=\mu_{\beta}(x)$; hence we may omit the suffix $\alpha$, and write $\mu_{\alpha}(x)=\mu(x)$. Again, we may suppose $\beta<\alpha$; then $x \in S_{\alpha}$, so that (15.3(ii)) $[x]=\mu(x)[x]$. By 12.14 , either $x=o$ or $\mu(x)=1$; in the latter case $[x]=[e]$, and so ( $e$ being bounded) $x=e$. Thus:

(2) If $\alpha \neq \beta, Q_{\alpha} \cap Q_{\beta}=\{o, e\}$.

15.4. The desired sub-algebra $P$ of $E$ will actually be $S_{\Omega}$; but it is more convenient to define it in another way. Let

$D=$ set of all elements $d$ expressible as $d=b_{1} b_{2} \cdots b_{k}$, where $b_{i} \in Q_{\alpha_{i}}$ and $\alpha_{1}>\alpha_{2}>\cdots>\alpha_{k}$;

$F=$ set of all elements $f$ expressible as $f=d_{1} \bigvee d_{2} \vee \cdots \vee d_{n}$, where $d_{i} \in D$ and the elements $d_{i}$ are disjoint;

$$
\begin{aligned}
& G=\left\{g \mid g=\bigvee d_{i}, d_{i} \in D\right\} \quad(i=1,2, \cdots) ; \\
& P=\left\{p \mid \phi=\wedge g_{n}, g_{n} \in G, g_{1} \geqq g_{2} \geqq \cdots\right\} .
\end{aligned}
$$

Clearly $D \subset F \subset G \subset P$. The first step consists in proving:

(3) If $x \in P, x=\mu(x)[e]$ for some real number $\mu(x)(0 \leqq \mu(x) \leqq 1) .(\mu(x)$ is then unique-from 12.14, Corollary.)

For suppose first $x=d \in D$, say $x=b_{1} b_{2} \cdots b_{k}$ as above. Then, since $b_{2} \cdots b_{k} \in S_{\alpha_{1}}, \quad[d]=\mu\left(b_{1}\right)\left[b_{2} \cdots b_{k}\right] \quad($ from $15.3(\mathrm{ii}))=\mu\left(b_{1}\right) \mu\left(b_{2}\right)\left[b_{3} \cdots b_{k}\right]$ $=\mu\left(b_{1}\right) \mu\left(b_{2}\right) \cdots \mu\left(b_{k}\right)[e]$, similarly; thus (3) holds in this case, with $\mu(d)$ $=\prod_{1}^{k} \mu\left(b_{i}\right)$.

Next, suppose $x=f \in F$. Then $f=\bigvee_{1}^{n} d_{i}$, where the elements $d_{i}$ are disjoint and belong to $D$; by the preceding, $\left[d_{i}\right]=\mu\left(d_{i}\right)[e]$; and then (12.16) $[f]$ $=\mu(f)[e]$ where $\mu(f)=\sum_{1}^{n} \mu\left(d_{i}\right)$.

Before considering $G$, we note that $F$ is a finitely additive algebra. In fact, it is clear that if $d_{1}, d_{2} \in D$, then $d_{1} d_{2} \in D$; and it readily follows that if $f$, $f^{\prime} \in F$, then $f f^{\prime} \in F$. Again, given $d \in D$, we see that $-d \in F$ as follows: We have $d=b_{1} b_{2} \cdots b_{k}$, say, where $b_{i} \in Q_{\alpha_{i}}$; consider the $2^{k}$ elements $d_{i}\left(1 \leqq i \leqq 2^{k}\right)$ of the form $c_{1} c_{2} \cdots c_{i}$ where each $c_{i}$ is either $b_{i}$ or $-b_{i}$. They are disjoint, and we may suppose $d=d_{1}$, say; then $-d=\bigvee d_{i}\left(2 \leqq i \leqq 2^{k}\right)$, showing that $-d \in F$ if $d \in D$. From the fact that $F$ is closed under (finite) infima, it now follows 
that $-f \in F$ if $f \in F$; for we have $f=\bigvee d_{i} \quad\left(d_{i} \in D\right)$, and consequently $-f=\Lambda\left(-d_{i}\right) \in F$.

It follows that each $g \in G$ is expressible as $g=\bigvee f_{n}$, where $f_{n} \in F$ and the elements $f_{n}$ are disjoint. (For if $g=\bigvee d_{i}, d_{i} \in D$, we take $f_{n}=\bigvee_{1}^{n} d_{i}-\bigvee_{1}^{n-1} d_{i}$.) From the preceding, we have $\left[f_{n}\right]=\mu\left(f_{n}\right)[e]$, and therefore $(12.16)[g]=\mu(g)[e]$ where $\mu(g)=\sum_{1}^{\infty} \mu\left(f_{n}\right)$.

Finally, given $p \in P$, we have $p=\Lambda g_{n}$, where $g_{1} \geqq g_{2} \geqq \cdots$, and, as just shown, $\left[g_{n}\right]=\mu\left(g_{n}\right)[e]$. By 12.19, $[p]=\mu(p)[e]$ where $\mu(p)=\lim _{n \rightarrow \infty} \mu\left(g_{n}\right)$.

Next,

(4) If $p_{n} \in P(n=1,2, \cdots)$, then $\bigvee_{p_{n}} \in P$.

For, from the definition of $P$, we have $p_{n}=\Lambda_{m} g_{n m}$, where $g_{n 1} \geqq g_{n 2} \geqq \ldots$. and $g_{n m} \in G$; and it has just been shown that $\mu\left(p_{n}\right)=\lim _{m \rightarrow \infty} \mu\left(g_{n m}\right)$. Hence for each $k$ we can choose $m=m(k, n)$ so that (i) $\mu\left(g_{n m}\right) \leqq \mu\left(p_{n}\right)+1 / 2^{n} k$, (ii) for each $k$ and $n, m$ is the least such number. Write $h_{k}=\mathrm{V}_{n} g_{n, m(k, n)}$; then $h_{k} \geqq \bigvee_{p_{n}}, h_{k} \in G$ (from the definition of $G$ ), and (from (ii)) $h_{1} \geqq h_{2} \geqq \cdots$. Further, 12.7 gives that $\left[h_{k}-V_{p_{n}}\right] \leqq(1 / k)[e]$, and so (12.6) $\bigvee_{p_{n}}=\wedge h_{k}$. Thus $\vee_{p_{n}} \in P$.

Again,

(5) If $p \in P$, then $-p \in P$.

For we have $p=\wedge g_{n}$, where $g_{1} \geqq g_{2} \geqq \cdots$ and $g_{n} \in G$. Thus $-p=\bigvee\left(-g_{n}\right)$, and by (4) it will suffice to prove that $-g_{n} \in P$, for each $n$. But $g_{n}=\bigvee_{i} d_{n i}$, say, where $d_{n i} \in D$. Hence $-g_{n}=\Lambda_{i}\left(-d_{n i}\right)=\Lambda_{m} x_{n m}$, where $x_{n m}=\Lambda\left(-d_{i}\right)$ $(1 \leqq i \leqq m)$. Thus $x_{n 1} \geqq x_{n 2} \geqq \cdots$, and $x_{n i} \in F$ (for $F$ is finitely additive) $\subset G$, so that $-g_{n} \in P$ by definition.

(4) and (5) show that $P$ is a $(\sigma-)$ sub-algebra of $E$; and (3), together with the properties in $\S 12$, shows that $\mu$ is a countably additive measure on $P$, $(P, \mu)$ being naturally isomorphic to $(P, \sim)$. Since $P \supset D \supset \cup Q_{\alpha}(1)$ gives $E=\mathscr{B}(P \cup U)$. Finally, the fact that $(P, \mu)$ is isometric to $I^{\mathfrak{m}}$ is an immediate consequence of the way in which the values of $\mu$ were derived successively on $\cup Q_{\alpha}, D, F, G$ and $P$; this shows (virtually by definition) that $(P, \mu)$ is (to within isometry) the direct product of all the measure algebras $Q_{\alpha}$, and those $Q_{\alpha}$ 's for which $Q_{\alpha}=L$ can obviously be disregarded.

\section{Inseparable case continued; proof of the principal lemma.}

16.1. Let $S$ be a sub-algebra of $E\left(=E\left(e_{n}\right)\right.$, as in $\left.\$ 15\right)$ such that (a) $S \supset U$, (b) every nonzero element of $E$ is of infinite order over $S$. The first part of the proof of $15.2(16.2-16.13)$ consists essentially in showing that $S$ has properties similar to those of $U$. Once this is done, the desired numerical measure algebra is constructed in much the same way as in the "separable case" ( $\$ 14)$.

16.2. Notation. For any $X \subset E$, write $X^{\prime}=X-(o)$. Thus, if $z \in S^{\prime}, S^{\prime}(z)$ denotes $\{y \mid y \in S$ and $o<y \leqq z\}$.

Throughout 16.2-16.10 we suppose a fixed element $b \in E$ to be given, and we define, for each $z \in S^{\prime}$, 


$$
\begin{aligned}
& \sigma(z)=\inf \{\eta \mid 0 \leqq \eta \leqq 1 \text { and } \eta[z] \geqq[b z]\}, \\
& \tau(z)=\sup \{\eta \mid 0 \leqq \eta \leqq 1 \text { and } \tau[z] \leqq[b z]\}
\end{aligned}
$$

Thus (from 12.14 and 12.19) we have

$$
\sigma(z)[z] \geqq[b z] \geqq \tau(z)[z]
$$

and

$$
0 \leqq \tau(z) \leqq \sigma(z) \leqq 1
$$

In the next few paragraphs, we derive some properties of the function $\sigma$; similar results and arguments will hold throughout for $\tau$. (The immediate goal is 16.7.)

16.3. If $z_{n} \in S^{\prime}(n=1,2, \cdots)$, and the elements $z_{n}$ are disjoint, then $\sigma\left(\mathrm{V} z_{n}\right) \leqq \sup \sigma\left(z_{n}\right)$.

For otherwise $\sigma\left(\mathrm{V} z_{n}\right)>\eta \geqq \sigma\left(z_{n}\right)$ for all $n$; and then $\eta\left[z_{n}\right] \geqq\left[b z_{n}\right]$, whence $\eta\left[\mathrm{V} z_{n}\right] \geqq\left[b\left(\mathrm{~V}_{n}\right)\right]$ and therefore $\eta \geqq \sigma\left(\mathrm{V}_{z_{n}}\right)$-a contradiction.

16.4. If $z \in S^{\prime}$ and $\sigma(z)>\eta \geqq 0$, there exists $z_{0} \in S^{\prime}(z)$ such that, for all $y \in S^{\prime}\left(z_{0}\right), \sigma(y) \geqq \eta$.

For if this is false, transfinite induction gives a countable set of disjoint elements $z_{\alpha}$ such that $\mathrm{V}_{\alpha}=z$ and $\sigma\left(z_{\alpha}\right)<\eta$. From $16.3, \sigma(z) \leqq \eta$-a contradiction.

16.5. Given $z_{0} \in S^{\prime}$ and $\epsilon>0$, there exists $y \in S^{\prime}\left(z_{0}\right)$ such that, whenever $y_{1}, y_{2} \in S^{\prime}(y)$, we have

$$
\left|\sigma\left(y_{1}\right)-\sigma\left(y_{2}\right)\right|<\epsilon \text { and }\left|\tau\left(y_{1}\right)-\tau\left(y_{2}\right)\right|<\epsilon .
$$

Write $\eta=\sup \left\{\sigma(z) \mid z \in S^{\prime}\left(z_{0}\right)\right\}$, and choose $z_{1} \in S^{\prime}\left(z_{0}\right)$ such that $\sigma\left(z_{1}\right)$ $>\eta-\epsilon$. We can suppose $\eta \neq 0$ (else we merely take $y=z_{0}$ ), and thus that $\eta-\epsilon>0$. On applying 16.4 to $z_{1}$, we obtain (say) $y^{1} \in S^{\prime}\left(z_{1}\right) \subset S^{\prime}\left(z_{0}\right)$ having the desired property as far as the function $\sigma$ is concerned. An "exhaustion" argument then gives $z_{0}=\bigvee y^{n}$, where $y^{n} \in S^{\prime}\left(z_{0}\right)$ and $\left|\sigma\left(y_{1}\right)-\sigma\left(y_{2}\right)\right|<\epsilon$ whenever $y_{1}, y_{2} \in S^{\prime}\left(y^{n}\right)$. An entirely similar argument applied to the function $\tau$ will give $z_{0}=\bigvee t^{n}$, where $t^{n} \in S^{\prime}\left(z_{0}\right)$ and $\left|\tau\left(y_{1}\right)-\tau\left(y_{2}\right)\right|<\epsilon$ whenever $y_{1}, y_{2} \in S^{\prime}\left(t^{n}\right)$. Since $z_{0}=\bigvee_{m, n} y^{m} t^{n}$, at least one of the elements $y^{m} t^{n}$ is nonzero, and gives the desired element $y$.

16.6. Given $z_{0} \in S^{\prime}$ and $\epsilon>0$, there exists $z \in S^{\prime}\left(z_{0}\right)$ such that $\left|\sigma\left(y_{1}\right)-\tau\left(y_{2}\right)\right|$ $<\epsilon$ whenever $y_{1}, y_{2} \in S^{\prime}(z)$.

By 16.5, there exists $y \in S^{\prime}\left(z_{0}\right)$ such that, whenever $y_{1}, y_{2} \in S^{\prime}(y), \mid \sigma\left(y_{1}\right)$ $-\sigma\left(y_{2}\right) \mid<\epsilon / 3$ and $\left|\tau\left(y_{1}\right)-\tau\left(y_{2}\right)\right|<\epsilon / 3$. We can suppose $y b \neq 0$ (for otherwise we merely take $z=y$ ). Now (13.1) there exist, for each positive integer $n, n$ disjoint invariant elements $u_{n i}(1 \leqq i \leqq n) \in U$ such that $\bigvee_{i} u_{n i}=e$ and

$$
((i-1) / n)\left[u_{n i}\right] \leqq\left[y b u_{n i}\right] \leqq(i / n)\left[u_{n i}\right]
$$

and similarly there exist $n$ disjoint elements $v_{n j} \in U(1 \leqq j \leqq n)$ such that 
$\mathrm{V}_{j} v_{n j}=e$ and

$$
((j-1) / n)\left[v_{n j}\right] \leqq\left[y v_{n j}\right] \leqq(j / n)\left[v_{n i}\right] .
$$

There will exist integers $n_{0}$ and $i_{0}$, with $n_{0} \geqq i_{0} \geqq 2$, such that $u_{n_{0} i_{0}} \neq 0$; for otherwise $[y b] \leqq(1 / n)[e]$ for all $n$, and thus (12.6) $y b=o$, contrary to our supposition. Choose a positive integer $p$ so large that $p\left(i_{0}-1\right)>2+6 / \epsilon$.

Write $N=n_{0} p$, and consider the elements $u_{N i}(1 \leqq i \leqq N)$. At least one of them, say $u_{N k}$, meets $u_{n_{0} i_{0}}$ (for $\bigvee_{i} u_{N i}=e$ ); and from (1), 12.13 and 12.14 we have $k / N \geqq\left(i_{0}-1\right) / n_{0}$, and so $k>2+6 / \epsilon$. Similarly $u_{N k}$ meets some $v_{N j}$; write $u_{N k} v_{N j}=u$, and $u y=z$. Thus $z \in S$ and $z \leqq y \leqq z_{0}$. From (1), (2) and 12.13 we have

$$
((k-1) / N)[u] \leqq[z b] \leqq(k / N)[u]
$$

and

$$
((j-1) / N)[u] \leqq[z] \leqq(j / N)[u] .
$$

Since $u \neq o$, it follows $(12.14$, Corollary) that $j \geqq k-1>1+6 / \epsilon$. Thus $z \neq 0$. Further, on using $12.5(6)$ we obtain $((k-1) / j)[z] \leqq((k-1) / N)[u] \leqq[z b]$, so that $(k-1) / j \leqq \tau(z)$. Again, $\sigma(z) \leqq k /(j-1)$; for this is trivial if $k>j-1$, and otherwise we have $[z b] \leqq(k /(j-1))((j-1) / N)[u] \leqq(k /(j-1))[z]$. Thus $0 \leqq \sigma(z)-\tau(z) \leqq k /(j-1)-(k-1) / j<\epsilon / 3$ (from the choice of $p)$. Finally, if $y_{1}, y_{2} \in S^{\prime}(z)$, we have $y_{1}, z \in S^{\prime}(y)$ and so $\left|\sigma\left(y_{1}\right)-\sigma(z)\right|<\epsilon / 3$; similarly $\left|\tau\left(y_{2}\right)-\tau(z)\right|<\epsilon / 3$, and the result follows.

16.7. Given $\epsilon>0$, there exists a disjoint sequence $\left\{z_{n}\right\}$ such that $z_{n} \in S^{\prime}$, $\bigvee z_{n}=e$, and $\left|\sigma\left(y_{1}\right)-\tau\left(y_{2}\right)\right|<\epsilon$ whenever $y_{1}, y_{2} \in S^{\prime}\left(z_{n}\right)$.

This follows from the preceding by "exhaustion."

16.8. Given a real number $\eta$ such that $0<\eta<1$, there exists $z \in S$ such that $\eta[y] \leqq[b y]$ for all $y \in S(z)$, and $\eta[t] \geqq[b t]$ for all $t \in S(-z)$.

On applying the preceding result, with $\epsilon=1 / n$, we obtain elements $z_{n i} \in S^{\prime}$ such that $z_{n i} z_{n j}=o$ if $i \neq j, \vee_{i} z_{n i}=e$, and $\left|\sigma\left(y_{1}\right)-\tau\left(y_{2}\right)\right| \leqq 1 / n$ whenever $y_{1}, y_{2} \in S^{\prime}\left(z_{n i}\right)$. We can further suppose that each $z_{(n+1) i}$ is less than or equal to some $z_{n j}$, since otherwise we replace (for each $n$ ) the elements $z_{n i}$ by all the nonzero elements of the form $z_{1 i_{1}} z_{2 i_{2}} \cdots z_{n i_{n}}$. Now let $I_{n}$ denote the set of values of $i$ (possibly empty) for which it is true that $\sigma(y) \leqq \eta$ whenever $y \in S\left(z_{n i}\right)$, and define

$$
s_{o}=o, \quad s_{n}=\bigvee\left\{z_{n i} \mid i \in I_{n}\right\} .
$$

Let $z=-\bigvee s_{n}$. Thus $s_{n} \in S, z \in S$, and $s_{1} \leqq s_{2} \leqq \cdots$. Hence if $t \in S(-z)$, we have $t=\mathrm{V}_{n}$ where $t_{n}=t\left(s_{n}-s_{n-1}\right) \quad(n=1,2, \cdots)$; thus $\sigma\left(t_{n}\right) \leqq \eta$, and therefore $(16.3) \sigma(t) \leqq \eta$, so that $\eta[t] \geqq[b t]$.

On the other hand, if $y \in S(z)$ then $y s_{n}=o$ for each $n$, and therefore $y=\bigvee\left\{y z_{n i} \mid i \notin I_{n}\right\}$. For each $z_{n i}$ occurring here, there exists (say) $r_{n i} \in S\left(z_{n i}\right)$ such that $\sigma\left(r_{n i}\right)>\eta$. Hence $\tau\left(y z_{n i}\right)>\eta-1 / n$, and therefore (from the property 
of $\tau$ analogous to 16.3$) \tau(y) \geqq \eta-1 / n$, so that $\tau(y) \geqq \eta$ and $\eta[y] \leqq[b y]$.

16.9. There exists a greatest $z$ having the properties stated in 16.8 .

Let $z_{0}$ be the supremum of all $z$ 's for which 16.8 holds. Then, from the countable chain condition, we have $z_{0}=\mathrm{V} z_{n}(n=1,2, \cdots)$, where 16.8 holds for each $z_{n}$. If $y \in S\left(z_{0}\right)$, we have $y=\bigvee y_{n}$ where $y_{1}=y z_{1}$ and $y_{n}=y\left(z_{n}\right.$ $\left.-\left(z_{1} \vee \cdots \vee z_{n-1}\right)\right)$. Thus $\eta\left[y_{n}\right] \leqq\left[b y_{n}\right]$, and it readily follows (cf. 12.17) that $\eta[y] \leqq[b y]$. If $t \in S\left(-z_{0}\right)$, then $t \in S\left(-z_{1}\right)$, and so $\eta[t] \geqq[b t]$. Thus 16.8 holds for $z_{0}$.

16.10. For each positive integer $n$, there exist $n$ disjoint elements $s_{1}, s_{2}, \ldots$, $s_{n} \in S$ such that (i) $\bigvee s_{i}=e$, (ii) whenever $z \in S\left(s_{i}\right),((i-1) / n)[z] \leqq[z b] \leqq(i / n)[z]$ $(1 \leqq i \leqq n)$.

(Compare 13.1.)

Using 16.8 and 16.9 , define $z_{i}$ to be the greatest element of $S$ such that $(i / n)[y] \leqq[b y]$ whenever $y \in S\left(z_{i}\right)$, and $(i / n)[t] \geqq[b t]$ whenever $t \in S\left(-z_{i}\right)$. Thus if $i<j$ we have $(i / n)\left[z_{j}-z_{i}\right] \geqq\left[b\left(z_{j}-z_{i}\right)\right] \geqq(j / n)\left[z_{j}-z_{i}\right]$, so that $z_{i}-z_{j}=o$; that is, $e \geqq z_{1} \geqq z_{2} \geqq \cdots \geqq z_{n}$. We have only to define $s_{1}=-z_{1}$, $s_{i}=z_{i-1}-z_{i}(1<i<n)$, and $s_{n}=z_{n-1}$.

(The maximality of $z_{i}$ has not been used, but will be convenient in the next paragraph.)

16.11. Given $c, d \in E$, there exists $z_{0} \in S$ such that $[c y] \leqq[d y]$ whenever $y \in S\left(z_{0}\right)$, and $[c t] \geqq[d t]$ whenever $t \in S\left(-z_{0}\right)$.

(Compare 7.6.)

Write $N=2^{n}$; then, for each $n(=1,2, \cdots)$ the preceding (applied with $b=c)$ gives $N$ disjoint elements $s_{n i}(1 \leqq i \leqq N) \in S$ such that $e=\bigvee_{i} s_{n i}$ and $((i-1) / N)[z] \leqq[z c] \leqq(i / N)[z]$ for all $z \in S\left(s_{n i}\right)$. Similarly there exist $2^{n}$ disjoint elements $t_{n j}(1 \leqq j \leqq N) \in S$ such that $e=\mathrm{V}_{j} t_{n j}$ and $((j-1) / N)[z]$ $\leqq[z d] \leqq(j / N)[z]$ for all $z \in S\left(t_{n j}\right)$. The method of construction used in 16.10 further shows that $s_{(n+1),(2 i-1)} \bigvee s_{(n+1), 2 i}=s_{n i}$, with a similar result for the $t$ 's. Thus, on writing $f_{n}=\bigvee\left\{s_{n i} t_{n j} \mid j \geqq i\right\}$, we have $f_{1} \geqq f_{2} \geqq \cdots$. Now define $z_{0}=\Lambda f_{n}$. It is not hard to verify that $z_{0}$ has the desired properties.

16.12. Given $b \in E^{\prime}$, there exists $y \in E^{\prime}(b)$ such that, for each $z \in S,[y z]$ $\leqq[b z] / 2$.

(Here we use for the first time the assumption that every element of $E^{\prime}$ has infinite order over $S$.)

Since $b$ is not of order 0 over $S$, there exists $h \leqq b$ such that $h \notin b S$.

Applying 16.11 to $c=h, d=b-h$, we obtain $z_{0} \in S$ such that [sh] $\leqq[s(b-h)]$ if $s \in S\left(z_{0}\right)$, and $[t h] \geqq[t(b-h)]$ if $t \in S\left(-z_{0}\right)$. Thus $[s h] \leqq(1 / 2)[s b]$ and $[t(b-h)] \leqq(1 / 2)[t b]$. Define $y=z_{0} h \bigvee\left(-z_{0}\right)(b-h)$. Thus clearly $y \leqq b$; and also $y \neq o$, since otherwise we obtain $b-z_{0}=h-z_{0}$ and $h z_{0}=o$, whence $h=b-z_{0}$ $\in b S$, a contradiction. Finally, given $z \in S$, we can write $y z=s h \vee t(b-h)$ where $s=z z_{0}$ and $t=z-z_{0}$. Hence $[y z]=(1 / 2)[s b \bigvee t b] \leqq(1 / 2)[b z]$

By iteration we now obtain:

16.12'. Corollary. Given $b \in E^{\prime}$ and $n>0$, there exists $y_{n}$ such that $o<y_{n}$ 
$\leqq b$ and, for each $z \in S,\left[y_{n} z\right] \leqq\left(1 / 2^{n}\right)[b z]$.

16.13. Let $b \in E, z \in S$, and $\eta(0<\eta<1)$ be such that $[b y] \geqq \eta[y]$ whenever $y \in S(z)$. Then there exists $b^{\prime} \leqq z b$ such that $\left[b^{\prime} y\right]=\eta[y]$ whenever $y \in S(z)$.

We can clearly suppose $z \neq 0$; thus $b z \neq 0$, and so $\left(16.12^{\prime}\right)$ there exists $c \in E^{\prime}(b z)$ such that, for each $y \in S,[c y] \leqq \eta[b y]$. Thus $c$ certainly satisfies: (i) $o<c \leqq b z$, (ii) for each $y \in S(z),[c y] \leqq \eta[y]$. An easy "exhaustion" argument now shows that there exists a maximal $c$, say $b^{\prime}$, satisfying (i) and (ii). Then $b^{\prime}$ is the desired element. For suppose not; then there exists $y \in S(z)$ such that $\left[b^{\prime} y\right]<\eta[y]$. From 16.10 we obtain, for each $n, n$ disjoint elements $s_{n i} \in S(1 \leqq i \leqq n)$ such that $\bigvee_{i} s_{n i}=e$ and, for all $s \in S\left(s_{n i}\right)$,

$$
((i-1) / n)[s] \leqq\left[s b^{\prime}\right] \leqq(i / n)[s] \text {. }
$$

Suppose first that, for every $n$, we have $y s_{n i}=o$ whenever $(i+1) / n<\eta$. It readily follows that $\left[b^{\prime} y\right] \geqq(\eta-2 / n)[y]$ for every $n$, and so $\left[b^{\prime} y\right] \geqq \eta[y]$, contrary to hypothesis. Thus we may choose $n$ and $i$ so that $(i+1) / n<\eta$ and $y s_{n i}=t$, say, $\neq 0$. Then, for every $s \in S(t)$, we have $\left[s b^{\prime}\right] \leqq(i / n)[s] \leqq(\eta-1 / n)[s]$, whence $\left[s\left(b-b^{\prime}\right)\right] \geqq(1 / n)[s]$.

It follows that $t\left(b-b^{\prime}\right) \neq o$, so that $\left(16.12^{\prime}\right)$ there exists $d \in E^{\prime}\left(t\left(b-b^{\prime}\right)\right)$ such that $[d s] \leqq(1 / n)\left[t\left(b-b^{\prime}\right) s\right] \leqq(1 / n)[t s]$ whenever $s \in S$. Define $c^{\prime}=b^{\prime} \bigvee d$; thus $o<c^{\prime} \leqq b$. Further, given any $y^{\prime} \in S(z)$, we have $c^{\prime} y^{\prime}=b^{\prime} y^{\prime} t \vee b^{\prime} y^{\prime}(-t)$ $\vee d y^{\prime} t$, a supremum of three disjoint elements in which

$$
\begin{aligned}
{\left[b^{\prime} y^{\prime} t\right] } & \leqq(\eta-1 / n)\left[y^{\prime} t\right], \\
\left.b^{\prime} y^{\prime}(-t)\right] & \leqq \eta\left[y^{\prime}(-t)\right] \quad\left(\text { from property (ii) of } b^{\prime}\right),
\end{aligned}
$$

and

$$
\left[d y^{\prime} t\right] \leqq(1 / n)\left[y^{\prime} t\right],
$$

from which we obtain $\left[c^{\prime} y^{\prime}\right] \leqq \eta\left[y^{\prime}\right]$, for all $y^{\prime} \in S(z)$. But this contradicts the maximality of $b^{\prime}$.

16.14. Given $x \notin S$, there exists elements $b(\rho) \in E\left(\rho=i / 2^{n}, 0 \leqq i \leqq 2^{n}\right.$, $n=0,1,2, \cdots)$ such that

(i) $[b(\rho) z]=\rho[z]$ whenever $z \in S$.

(ii) $b\left(\rho_{1}\right) \leqq b\left(\rho_{2}\right)$ if $\rho_{1} \leqq \rho_{2} ; b(0)=o$, and $b(1)=e$.

(iii) $x \in \mathcal{B}(S \cup B)$, where $B=\bigcup(b(\rho))$.

For each $\rho=i / 2^{n}\left(0 \leqq i \leqq 2^{n}, n \geqq 0\right)$, we define $s(\rho)$ (using 16.9) to be the greatest element of $S$ such that

$$
\begin{aligned}
& {[x z] \geqq \rho[z] \quad \text { whenever } \quad z \in S(s(\rho)), \text { and }} \\
& {[x z] \leqq \rho[z] \quad \text { whenever } \quad z \in S(-s(\rho)) .}
\end{aligned}
$$

Thus (cf. 16.10) we have

(2) If $\rho_{1} \leqq \rho_{2}, e=s(0) \geqq s\left(\rho_{1}\right) \geqq s\left(\rho_{2}\right)$, and $s(1) \leqq x$.

The elements $b\left(i / 2^{n}\right)$ are defined by induction over $n$, starting with 
$b(0) \equiv b\left(0 / 2^{0}\right)=o$, and $b(1) \equiv b\left(1 / 2^{0}\right)=e$. Suppose that $n \geqq 1$, and that $b\left(i / 2^{m}\right)$ has been defined for all $m<n$ and $i \leqq 2^{m}$ in such a way that properties (i) and (ii) (of the statement of the present proposition) hold, and so that, in addition, we have

$$
b(\rho) s(\rho) \leqq x, \text { and } x-s(\rho) \leqq b(\rho)
$$

for all $\rho=i / 2^{m}, m<n$. (This will trivially be the case when $n=1$.) We define $b\left(2 k / 2^{n}\right)=b\left(k / 2^{n-1}\right)$, so that the definition is consistent. The definition of $b\left((2 k-1) / 2^{n}\right)$ is more troublesome, and in giving it we suppose (to save suffixes) that $k$ and $n$ are fixed $\left(1 \leqq k \leqq 2^{n-1}\right)$, and write $\rho^{-}=(k-1) / 2^{n-1}$, $\rho^{0}=(2 k-1) / 2^{n}$, and $\rho^{+}=k / 2^{n-1}$. We use $s_{1}, s_{2}$ and $s_{3}$ to denote (arbitrary) elements of $S\left(-s\left(\rho^{0}\right)\right), S\left(s\left(\rho^{0}\right)-s\left(\rho^{+}\right)\right)$and $S\left(s\left(\rho^{+}\right)\right)$, respectively.

First we show

$$
\left[s_{1} x-b\left(\rho^{-}\right)\right] \leqq\left(1 / 2^{n}\right)\left[s_{1}\right] .
$$

For $s_{1}=z^{\prime} \bigvee z^{\prime \prime}$ where $z^{\prime}=s_{1}-s\left(\rho^{-}\right)$and $z^{\prime \prime}=s_{1}\left\{s\left(\rho^{-}\right)-s\left(\rho^{0}\right)\right\}$. Now $\left[z^{\prime \prime} x\right]$ $\leqq \rho^{0}\left[z^{\prime \prime}\right]$ from (1); $\left[z^{\prime \prime} x b\left(\rho^{-}\right)\right]=\left[z^{\prime \prime} b\left(\rho^{-}\right)\right]=\rho^{-}\left[z^{\prime \prime}\right]$ from (3) and (i); and $z^{\prime} x-b\left(\rho^{-}\right)=o$ from (3). (4) now follows easily.

A fortiori, $\left[s_{1} x\left\{b\left(\rho^{+}\right)-b\left(\rho^{-}\right)\right\}\right] \leqq\left(1 / 2^{n}\right)\left[s_{1}\right]$; and since $\left[s_{1}\left\{b\left(\rho^{+}\right)-b\left(\rho^{-}\right)\right\}\right]$ $=\left(1 / 2^{n-1}\right)\left[s_{1}\right]\left(\right.$ from $(\mathrm{i})$ and (ii)), we have $\left[s_{1}(-x)\left\{b\left(\rho^{+}\right)-b\left(\rho^{-}\right)\right\}\right] \geqq\left(1 / 2^{n}\right)\left[s_{1}\right]$. Here $s_{1}$ is any element of $S\left(-s\left(\rho^{0}\right)\right)$. Thus, from 16.13 , there exists $y_{0} \leqq$ $(-x)\left\{b\left(\rho^{+}\right)-b\left(\rho^{-}\right)\right\}-s\left(\rho^{0}\right)$ such that $\left[y_{0} s_{1}\right]=\left(1 / 2^{n}\right)\left[s_{1}\right]$ for all $s_{1}\left(\in S\left(-s\left(\rho^{0}\right)\right)\right)$. Write $y_{1}=\left\{b\left(\rho^{+}\right)-b\left(\rho^{-}\right)\right\}\left(-s\left(\rho^{0}\right)\right)-y_{0}$. Thus for all $s_{1}$ we have (on using (i) and (ii))

$$
\left\lfloor y_{1} s_{1}\right\rfloor=\left(1 / 2^{n}\right)\left[s_{1}\right\rfloor .
$$

Similar arguments prove the existence of $y_{2} \leqq x\left\{b\left(\rho^{+}\right)-b\left(\rho^{-}\right)\right\}\left\{s\left(\rho^{0}\right)\right.$ $\left.-s\left(\rho^{+}\right)\right\}$such that

$$
\left[y_{2} s_{2}\right]=\left(1 / 2^{n}\right)\left[s_{2}\right] \quad\left(s_{2} \in S\left\{s\left(\rho^{0}\right)-s\left(\rho^{+}\right)\right\}\right),
$$

and of $y_{3} \leqq\left\{b\left(\rho^{+}\right)-b\left(\rho^{-}\right)\right\} s\left(\rho^{+}\right) \leqq x$ such that

$$
\left[y_{3} s_{3}\right]=\left(1 / 2^{n}\right)\left[s_{3}\right] \quad\left(s_{3} \in S\left(s\left(\rho^{+}\right)\right)\right) .
$$

Define $y=y_{1} \bigvee y_{2} \bigvee y_{3}$, and $b\left(\rho^{0}\right)=b\left(\rho^{-}\right) \bigvee y$. Clearly property (ii) is maintained. To establish (i), it will suffice to verify that $[y s]=\left(1 / 2^{n}\right)[s]$ for all $s \in S$. But we can write $s=s_{1} \bigvee s_{2} \bigvee s_{3}$; and it is easily verified that $y s_{i}=y_{i} s_{i}$ $(i=1,2,3)$. Since the elements $s_{i}$ are necessarily disjoint, the desired relation follows from (5), (6) and (7). Property (3) can likewise be verified, and the inductive definition of the elements $b(\rho)$ is complete.

Finally, to prove (iii), we show

$$
x=\mathrm{V} x_{n}, \quad \text { where } \quad x_{n}=\bigvee b\left(i / 2^{n}\right) s\left(i / 2^{n}\right)
$$

From (3), $x \geqq x_{n}$ for every $n$. Now 


$$
\begin{aligned}
x-x_{n} & \leqq x-x\left\{\bigvee_{i=0}^{2^{n}-1} b\left(i / 2^{n}\right)\left\{s\left(i / 2^{n}\right)-s\left((i+1) / 2^{n}\right)\right\}\right\} \\
& =\bigvee_{0}^{2^{n}-1}\left\{x-b\left(i / 2^{n}\right)\right\}\left\{s\left(i / 2^{n}\right)-s\left((i+1) / 2^{n}\right)\right\} \\
& \left.=\mathrm{V}_{i}, \text { say. } \quad\left(\text { since } x=\bigvee_{i} x\left\{s\left(i / 2^{n}\right)-s(i+1) / 2^{n}\right)\right\}\right)
\end{aligned}
$$

But from (1), $\left[x\left\{s\left(i / 2^{n}\right)-s\left((i+1) / 2^{n}\right)\right\}\right] \leqq\left((i+1) / 2^{n}\right)\left[s\left(i / 2^{n}\right)-s\left((i+1) / 2^{n}\right)\right]$; and, from (3) and (i), $\left[x b\left(i / 2^{n}\right)\left\{s\left(i / 2^{n}\right)-s\left((i+1) / 2^{n}\right)\right\}\right]=\left(i / 2^{n}\right)\left[s\left(i / 2^{n}\right)\right.$ $\left.-s\left((i+1) / 2^{n}\right)\right]$. Hence $\left.\left[t_{i}\right] \leqq\left(1 / 2^{n}\right)\left[s\left(i / 2^{n}\right)-s(i+1) / 2^{n}\right)\right]$, and so $(12.5(5))$ $\left[x-x_{n}\right] \leqq\left(1 / 2^{n}\right)[e]$. That is, $\left[x-\bigvee_{x_{n}}\right] \leqq\left(1 / 2^{n}\right)[e]$ for every $n$; and therefore (12.6) $x=\mathrm{V} x_{n}$, completing the proof.

16.15. The "principal lemma" (15.2). In 16.14, let $Q=\mathbb{B}(B)$; then $(Q, \sim)$ is naturally isomorphic to a numerical measure algebra $(Q, \mu)$, in such a way that, for each $q \in Q$ and $s \in S,[q s]=\mu(q)[s]$. Further, $(Q, \mu)$ is isometric to $I^{1}$.

This following from 16.14 by essentially the same arguments as in the "separable case" (14.5 and 14.6), the elements $b\left(i / 2^{n}\right)$ here playing the role of the elements $d_{\rho}$ in $\$ 14$.

17. $E\left(e_{n}\right)$ as a direct product.

17.1. As in $\S \S 14,15$, we consider the relative algebra $E\left(e_{n}\right), e_{n}$ being any one of the elements of 13.4. The theorems of 14.1 and 15.1 show that, in every case, $E\left(=E\left(e_{n}\right)\right)=\mathscr{B}(P \cup U)$, where $P$ is a (relative) sub-algebra naturally isomorphic with $I^{1}$ or $I^{\mathfrak{m}}$, in such a way that (from 12.13) we have the fundamental relation:

$[p u]=\mu(p)[u]$ whenever $p \in P$ and $u \in U$. $\$ 4)$.

We shall now deduce that $E$ is isomorphic to the direct product $P \otimes U$ (cf.

17.2. Definitions. Let $R, S$ denote the respective representation spaces of $P$ and $U$; as in 4.2 , we use $p^{*}, u^{*}$ to denote the open-closed subsets of $R, S$ respectively which correspond to $p \in P, u \in U$. Let $Q^{*}$ denote the class of all subsets $Y$ of $R \times S$ which are expressible in the form $Y=U\left(p_{i}^{*} \times u_{i}^{*}\right)$, where the (open-closed) sets $u_{i}^{*}$ are disjoint and their union is residual in $S$ (that is, $S-U u_{i}{ }^{*}$ is of first category). Similarly let $Q$ denote the class of all elements $y \in E$ expressible as $y=\bigvee_{p_{i}} u_{i}$, where $p_{i} \in P, u_{i} \in U$, the elements $u_{i}$ are disjoint, and $\mathrm{V} u_{i}=e$.

We observe that $Q$ is a finitely additive sub-algebra of $E$. In fact, if $y_{1}, y_{2} \in Q$ and $y_{j}=\bigvee_{i} p_{i j} u_{i j}$ as above, then $-y_{1}=\mathrm{V}\left(-p_{i 1}\right) u_{i 1} \in Q$ and $y_{1} \bigvee y_{2}$ $=\bigvee_{i, k}\left(p_{i 1} \bigvee p_{k 2}\right)\left(u_{i 1} u_{k 2}\right) \in Q$. Similarly $Q^{*}$ is a finitely additive field of sets.

If $y \in Q$ is expressed as above in two ways, say $y=V_{p_{i}} u_{i}=V_{q_{j}} v_{j}$ (where $p_{i}, q_{j} \in P ; u_{i}, u_{j} \in U ; \bigvee u_{i}=e=\bigvee v_{j}$, and each of the sequences $u_{i}, v_{j}$, is disjoint), then the "corresponding" sets $U\left(p_{i}^{*} \times u_{i}^{*}\right)$ and $U\left(q_{j}^{*} \times v_{j}^{*}\right)$ in $Q^{*}$ are equal. For we have $\left(p_{i}+{ }_{2} q_{j}\right) u_{i} v_{j}=\left(y+{ }_{2} y\right) u_{i} v_{j}=o$, so (from the fundamental 
property of $P$ ) $\mu\left(p_{i}+{ }_{2} q_{j}\right)\left[u_{i} v_{j}\right]=o$, and therefore $p_{i}=q_{j}$ whenever $u_{i} v_{j} \neq 0$. Thus $p_{i}^{*}=q_{j}^{*}$ whenever $u_{i}^{*} \bigcap v_{j}^{*} \neq 0$, so that

$$
\bigcup\left(p_{i}^{*} \times u_{i}^{*}\right)=\bigcup_{i, j} p_{i}^{*} \times\left(u_{i}^{*} \cap v_{j}^{*}\right)=\bigcup_{i, j} q_{j}^{*} \times\left(u_{i}^{*} \cap v_{j}^{*}\right)=\bigcup_{j} q_{j}^{*} \times v_{j}^{*} .
$$

Hence we may define a mapping $\phi_{1}$ of $Q$ in $Q^{*}$ by setting $\phi\left(V_{p_{i}} u_{i}\right)$ $=\mathrm{U}\left(p_{i}^{*} \times u_{i}^{*}\right)$. An argument similar to the preceding shows that $\phi_{1}$ is $1-1$; and $\phi_{1}$ is evidently a finitely additive algebraic isomorphism between $Q$ and Q*. 4.3)

We have ( $M$ denoting the continuous-function "measure" in $P \otimes U-\mathrm{cf}$.

(1) If $Y=\phi_{1}(y)\left(y \in Q, Y \in Q^{*}\right)$, then $[y] \leqq \epsilon[e] \rightleftarrows M\{Y\} \leqq \epsilon(0<\epsilon<1)$.

For suppose $y=V_{p_{i}} u_{i}$ in "normal form," as above. Then if $[y] \leqq \epsilon[e]$ we have (12.13) $\left[u_{i} y\right] \leqq \epsilon\left[u_{i}\right]$; but $\left[u_{i} y\right]=\left[u_{i} p_{i}\right]=\mu\left(p_{i}\right)\left[u_{i}\right]$, and thus (12.14) $\mu\left(p_{i}\right) \leqq \epsilon$ whenever $u_{i} \neq 0$. Thus $M\{Y\} \leqq \epsilon$ (for each $s \in S$ ). The converse implication is proved similarly.

17.3. The desired isomorphism between $E$ and $P \otimes U$ is now obtained by "extending" $\phi_{1}$, as follows. Suppose two elements $x \in E$ and $X \in P \otimes U$ are so related, that to every $\epsilon>0$ (and $<1$ ) there correspond $y \in Q$ and $Y \in Q^{*}$ such that $\left[x+{ }_{2} y\right] \leqq \epsilon[e], M\left\{X+{ }_{2}\{Y\}\right\} \leqq \epsilon$, and $Y=\phi_{1}(y)$. We then write $X=\phi(x)$. It is easily seen from (1) that this relation is $1-1$ (where it exists); and clearly if $x \in Q$ then $\phi(x)$ exists and equals $\left\{\phi_{1}(x)\right\}$. Before proving that $\phi$ is defined for all $x \in E$, we need some lemmas.

17.4. Let $y=\mathrm{V} y_{n}$, where $y_{n} \in Q(n=1,2, \cdots)$. Then given $\epsilon>0$ (and $<1)$, there exists $z \in Q$ such that $\left[y+{ }_{2} z\right] \leqq \epsilon[e]$.

We may suppose that $y_{1} \leqq y_{2} \leqq \cdots$ (on replacing $y_{n}$ by $\bigvee_{1}^{n} y_{i}$ ). By 7.6 there exists for each $n$ an invariant element $w_{n}$ such that (i) $\left[\left(y-y_{n}\right) w_{n}\right] \leqq \epsilon\left[w_{n}\right]$, (ii) if $v$ is a nonzero invariant element less than or equal to $-w_{n},\left[\left(y-y_{n}\right) v\right]$ $>\epsilon[v]$. It readily follows that $w_{1} \leqq w_{2} \leqq \cdots$; further, $V w_{n}=e$, since if $e-V w_{n}$ $=v_{0}$, we have $\left[y-y_{n}\right] \geqq\left[\left(y-y_{n}\right) v_{0}\right] \geqq \epsilon\left[v_{0}\right]$ for every $n$, whence $(7.2)[o] \geqq \epsilon\left[v_{0}\right]$, and so (12.14, Corollary) $v_{0}=o$. Now write $v_{1}=w_{1}, v_{n}=w_{n}-w_{n-1}(n \geqq 2)$, so that the elements $v_{n}$ are disjoint and $V v_{n}=e$; and define $z=\mathrm{V} y_{n} v_{n}$. Then $y+{ }_{2} z=\mathrm{V}\left(y+{ }_{2} y_{n}\right) v_{n}=\mathrm{V}\left(y-y_{n}\right) v_{n}$ where, since $v_{n} \leqq w_{n}$, we have $\left[\left(y-y_{n}\right) v_{n}\right]$ $\leqq \epsilon\left[v_{n}\right]$. Thus (12.17) $\left[y+{ }_{2} z\right] \leqq \epsilon[e]$. Finally, since $y_{n} \in Q$, we may write $y_{n}=\mathrm{V}_{i} p_{n i} u_{n i}$ in "standard form"; and then $z=\bigvee_{n, i} p_{n i}\left(u_{n i} v_{n}\right) \in Q$. $\leqq \epsilon[e]$.

17.5. Given $x \in E$ and $\epsilon>0$ (and $<1$ ), there exists $y \in Q$ such that $\left[x+{ }_{2} y\right]$

Let $B^{\prime}$ be the set of those elements $x \in E$ for which the above statement is true (for all $\epsilon$ ). Evidently $B^{\prime} \supset Q \supset P \cup U$; also if $x \in B^{\prime}$ so does $-x$, and if $x_{n} \in \mathcal{B}^{\prime}$ then $\mathrm{V} x_{n} \in \mathcal{B}^{\prime}$, as readily follows from 17.4. Thus $\mathcal{B}^{\prime} \supset \mathcal{B}(P \cup U)=E$.

17.6. If $y_{n} \in E$ and $\left[y_{m}+{ }_{2} y_{n}\right] \leqq\left(1 / 2^{n}\right)[e]$ whenever $m \geqq n$, there exists $y \in E$ such that $\left[y+{ }_{2} y_{n}\right] \leqq\left(1 / 2^{n-2}\right)[e]$ for all $n \geqq 2$; further, this $y$ is unique, and is given by $y=\Lambda_{n} \bigvee_{i \geqq n} y_{i}\left(=\lim \sup y_{i}\right)$. 
REMARK. On complementation we obtain that $y$ is also given by $y=\Lambda_{n} \bigvee_{i \geqq n} y_{i}=\lim$ inf $y_{i}$; thus the assertion is, roughly speaking, that "Cauchy sequences" converge.

Write $\quad t_{n}=y_{n}+{ }_{2} \mathrm{~V}_{i \geqq n} y_{i}=\mathrm{V}_{i \geqq n}\left(y_{i}-y_{n}\right)=\mathrm{V}_{i \geqq n}\left(y_{i+1}-y_{i} y_{i+1}\right) \leqq \mathrm{V}_{i \geqq n}\left(y_{i+1}\right.$ $\left.+{ }_{2} y_{i}\right)$. Thus (12.7) $\left[t_{n}\right] \leqq\left(1 / 2^{n-1}\right)[e]$. Now define $y=\Lambda_{n} \bigvee_{i \geqq n} y_{i}$. Then $y=\Lambda_{n \geqq N} \bigvee_{i \geqq n} \quad y_{i}=\Lambda_{n \geqq N}\left(y_{n}+{ }_{2} t_{n}\right)$ so that, for each $N, y_{N}+{ }_{2} y \leqq\left\{y_{N} \vee \Lambda_{n} \leqq N\right.$ $\left.\left(y_{n} \bigvee t_{n}\right)\right\}-\left\{y_{N} \wedge \bigwedge_{n \geqq N}\left(y_{n}-t_{n}\right)\right\} \leqq\left[y_{N} \bigvee \bigvee_{n \geqq N} t_{n}\right\}-\left\{y_{N}-\bigvee_{n \geqq N} t_{n}\right\}=\bigvee_{n} \geqq N t_{n}$. Hence (12.7 again) $\left[y_{N}+{ }_{2} y\right] \leqq\left(1 / 2^{N-2}\right)[e]$ for all $N>1$. Finally, $y$ is unique, since if $z$ also has the same property we have $\left[y+{ }_{2} z\right] \leqq\left(1 / 2^{n-3}\right)[e]$ for all $n \geqq 3$, and so (12.6) $y=z$.

17.7. $\phi$ is a finitely additive algebraic isomorphism between $E$ and $P \otimes U$.

Given $x \in E$, we first show that $\phi(x)$ exists. From 17.5, there exists a sequence $y_{n} \in Q$ with $\left[x+{ }_{2} y_{n}\right] \leqq\left(1 / 2^{n+2}\right)[e]$. By $17.2(1)$, the corresponding sets $Y_{n} \in Q^{*}$ (that is, $\left.Y_{n}=\phi_{1}\left(y_{n}\right)\right)$ satisfy $M\left\{Y_{m}+{ }_{2} Y_{n}\right\} \leqq 1 / 2^{n}$ whenever $m \geqq n$. Hence, by 17.6 applied to the algebra $P \otimes U$, there exists $X \in P \otimes U$ such that $M\left\{X+{ }_{2}\left\{Y_{n}\right\}\right\} \leqq 1 / 2^{n-2}$. Clearly $X=\phi(x)$, by definition (17.3).

A similar argument (starting from 4.6) shows that $\phi^{-1}(X)$ exists for every $X \in P \otimes U$. Finally, it has already been pointed out that $\phi$ is $1-1$, and the relations $\phi(-x)=-\phi(x), \phi\left(x_{1} \bigvee x_{2}\right)=\phi\left(x_{1}\right) \bigvee \phi\left(x_{2}\right)$ are obvious.

17.8. If $y_{n} \in Q, \phi\left(\bigvee y_{n}\right)=\bigvee_{\phi}\left(y_{n}\right)$.

It will suffice to prove this assuming $y_{1} \leqq y_{2} \leqq \cdots$. Given $\epsilon>0$, consider the element $z \in Q$ constructed in 17.4. Thus $\left[y+{ }_{2} z\right] \leqq \epsilon[e]$, where $y=\bigvee y_{n}$; further, the construction shows that $z=\bigvee y_{n} v_{n}$, where $v_{n} \in U, \bigvee v_{n}=e$, and the elements $v_{n}$ are disjoint. Thus $z v_{n} \leqq y_{n}$. Now write $Y_{n}=\phi_{1}\left(y_{n}\right), Z=\phi_{1}(z)$; we have $Z \cap\left(R \times v_{n}^{*}\right) \subset Y_{n}$, so that $Z \subset \cup Y_{n} \cup(R \times H)$ where $H$ is of the first category in $S$.

Now, we have from 17.4 that $\left[y v_{n}-z v_{n}\right] \leqq \epsilon\left[v_{n}\right]$; a fortiori, therefore, $\left[y_{m} v_{n}-z v_{n}\right] \leqq \epsilon\left[v_{n}\right]$ when $m \geqq n$, so that $(17.2(1)) \quad M\left\{\left(Y_{m} \cap\left(R \times v_{n}^{*}\right)\right)\right.$ $\left.-\left(Z \cap\left(R \times v_{n}^{*}\right)\right)\right\} \leqq \epsilon M\left\{R \times v_{n}^{*}\right\}$. The countable additivity of $M$ then gives (on summing first over $m$ and then over $n$ )

$$
M\left\{\cup Y_{n}-Z\right\} \leqq \epsilon .
$$

Thus $M\left\{\cup Y_{n}+{ }_{2} Z\right\} \leqq \epsilon$; and so $\phi(y)=\left\{\cup Y_{n}\right\}$ from the definition.

17.9. If $x_{n} \in E, \phi\left(\bigvee x_{n}\right)=\mathrm{V} \phi\left(x_{n}\right)$.

As before, we may suppose that $x_{1} \leqq x_{2} \leqq \ldots$. Let $\epsilon$ be given, and choose $y_{n} \in Q$ so that $\left[x_{n}+{ }_{2} y_{n}\right] \leqq\left(\epsilon / 2^{n}\right)[e]$ and $M\left\{\phi\left(x_{n}\right)+{ }_{2} \phi\left(y_{n}\right)\right\} \leqq \epsilon / 2^{n}$ (using the definition of $\phi)$. Write $x=\mathrm{V} x_{n}, y=\mathrm{V} y_{n}$. It is easily verified that $x+{ }_{2} y$ $\leqq \mathrm{V}\left(x_{n}+{ }_{2} y_{n}\right)$, so that $\left[x+{ }_{2} y\right] \leqq \epsilon[e]$. Similarly $M\left\{X+{ }_{2} Y\right\} \leqq \epsilon$, where $X=\mathrm{V}_{\phi}\left(x_{n}\right), \quad Y=\mathrm{V}_{\phi}\left(y_{n}\right)$. But 17.8 shows that $Y=\phi(y)$; hence there exists $z \in Q$ such that $\left[y+{ }_{2} z\right] \leqq \epsilon[e]$ and $M\left\{Y+{ }_{2}\{Z\}\right\} \leqq \epsilon$, where $Z=\phi_{1}(z)$. Thus $\left[x+{ }_{2} z\right] \leqq(2 \epsilon)[e]$ and $M\left\{X+{ }_{2}\{Z\}\right\} \leqq 2 \epsilon$, showing that $X=\phi(x)$.

This completes the proof that $\phi$ is an algebraic $(\sigma-)$ isomorphism between $E$ and $J \otimes U$. To prove that $\phi$ is an isomorphism of the abstract meas- 
ure algebras, we must show that $\phi$ is "measure-preserving" both ways.

17.10. If $x_{1} \sim x_{2}, M\left\{\phi\left(x_{1}\right)\right\}=M\left\{\phi\left(x_{2}\right)\right\}$.

Given $\epsilon>0$ (and $<1)$, we choose $y_{i} \in Q(i=1,2)$ so that $\left[x_{i}+{ }_{2} y_{i}\right] \leqq \epsilon[e]$ and $M\left\{X_{i}+{ }_{2}\left\{Y_{i}\right\}\right\} \leqq \epsilon$, where $X_{i}=\phi\left(x_{i}\right)$ and $Y_{i}=\phi_{1}\left(y_{i}\right)$. Let $y_{1}=\mathrm{V}_{p_{n}} u_{n}$, $y_{2}=\mathrm{V} q_{n} v_{n}$, where (as usual) $p_{n}, q_{n} \in P, u_{n}, v_{n} \in U, \bigvee u_{n}=e=\bigvee v_{n}$, and the sequences $\left\{u_{n}\right\},\left\{v_{n}\right\}$ are each disjoint. We can further suppose $u_{n}=v_{n} \neq o$ (on replacing both sequences $u_{n}, v_{n}$ by an enumeration of the nonzero elements $\left.u_{n} v_{m}\right)$.

First we show

$$
\mu\left(p_{n}\right) \leqq \mu\left(q_{n}\right)+2 \epsilon .
$$

For we can suppose $\mu\left(q_{n}\right)+2 \epsilon \leqq 1$, else there is nothing to prove. Now, since $x_{1} \sim x_{2}$, there exists $t \leqq x_{2}$ such that $t \sim x_{1} y_{1}$. For each $n$ we have $\left[u_{n} x_{1} y_{1}\right]=\left[u_{n} t\right] \leqq\left[u_{n} y_{2} \bigvee u_{n}\left(x_{2}-y_{2}\right)\right]$, where $\left[u_{n} y_{2}\right]=\left[u_{n} q_{n}\right]=\mu\left[\left(q_{n}\right)\left[u_{n}\right]\right.$, and $\left[u_{n}\left(x_{2}-y_{2}\right)\right] \leqq \epsilon\left[u_{n}\right]$. Thus $\left[u_{n} x_{1} y_{1}\right] \leqq\left(\mu\left(q_{n}\right)+\epsilon\right)\left[u_{n}\right]$. Since further $\left[u_{n}\left(y_{1}-x_{1}\right)\right]$ $\leqq \epsilon\left[u_{n}\right]$, we have $\left[u_{n} y_{1}\right] \leqq\left(\mu\left(q_{n}\right)+2 \epsilon\right)\left[u_{n}\right]$. But $\left[u_{n} y_{1}\right]=\left[u_{n} p_{n}\right]=\mu\left(p_{n}\right)\left[u_{n}\right]$; and (1) follows.

A similar argument applies with $p_{n}$ and $q_{n}$ interchanged. Thus we evidently have

$$
\left|M\left\{Y_{1}\right\}-M\left\{Y_{2}\right\}\right| \leqq 2 \epsilon .
$$

But $M\left\{X_{i}\right\}=M\left\{X_{i}\left\{Y_{i}\right\}\right\}+M\left\{X_{i}-\left\{Y_{i}\right\}\right\}=M\left\{Y_{i}\right\}-M\left\{\left\{Y_{i}\right\}-X_{i}\right\}$ $+M\left\{X_{i}-\left\{Y_{i}\right\}\right\}$, so that $\left|M\left\{X_{i}\right\}-M\left\{Y_{i}\right\}\right| \leqq \epsilon$. Hence $\left|M\left\{X_{1}\right\}-M\left\{X_{2}\right\}\right|$ $\leqq 4 \epsilon$, and therefore

$$
M\left\{X_{1}\right\}=M\left\{X_{2}\right\} .
$$

17.11. If $M\left\{\phi\left(x_{1}\right)\right\}=M\left\{\phi\left(x_{2}\right)\right\}$, then $x_{1} \sim x_{2}$.

Given $\epsilon(0<\epsilon<1 / 9)$, we take $y_{1}, y_{2} \in Q$ so that $\left[x_{i}+{ }_{2} y_{i}\right] \leqq \epsilon[e]$ and $M\left\{X_{i}+{ }_{2}\left\{Y_{i}\right\}\right\} \leqq \epsilon$, where $X_{i}=\phi\left(x_{i}\right)$ and $Y_{i}=\phi_{1}\left(y_{i}\right), i=1,2$. As in the previous argument, we can suppose $y_{1}=\bigvee_{p_{n}} u_{n}, y_{2}=\bigvee q_{n} u_{n}$, where $p_{n}, q_{n} \in P, u_{n} \in U$, $u_{n} \neq o$, and the sequence $u_{n}$ is disjoint. As at the end of 17.10 , we have $\left|M\left\{X_{i}\right\}-M\left\{Y_{i}\right\}\right| \leqq \epsilon$ and therefore $\left|M\left\{Y_{1}\right\}-M\left\{Y_{2}\right\}\right| \leqq 2 \epsilon$; and it readily follows that $\left|\mu\left(p_{n}\right)-\mu\left(q_{n}\right)\right| \leqq 2 \epsilon$, for each $n$. We may therefore choose $p_{n}^{\prime} \in P\left(p_{n}\right), q_{n}^{\prime} \in P\left(q_{n}\right)$ so that $\mu\left(p_{n}^{\prime}\right)=\mu\left(q_{n}^{\prime}\right), \mu\left(p_{n}\right)-\mu\left(p_{n}^{\prime}\right) \leqq 2 \epsilon$, and $\mu\left(q_{n}\right)$ $-\mu\left(q_{n}^{\prime}\right) \leqq 2 \epsilon$. Define $y_{1}^{\prime}=\bigvee p_{n}^{\prime} u_{n}$ and $y_{2}^{\prime}=\bigvee q_{n}^{\prime} u_{n}$; thus $y_{1}^{\prime} \sim y_{2}^{\prime}, y_{i}^{\prime} \leqq y_{i}$, and $\left[y_{i}-y_{i}^{\prime}\right] \leqq(2 \epsilon)[e]$. Hence $\left.\left[x_{i}+{ }_{2}\right]_{i}^{\prime}\right] \leqq(3 \epsilon)[e]$.

Since $y_{1}^{\prime} \sim y_{2}^{\prime}$, we can write $y_{2}^{\prime}=t \bigvee z_{1}$, where $t \sim x_{1} y_{1}^{\prime}$ and $z_{1} \sim y_{1}^{\prime}-x_{1}$, so that $\left[z_{1}\right] \leqq(3 \epsilon)[e]$; and we then have $y_{2}^{\prime}=t x_{2} y_{2}^{\prime} \bigvee z_{2}$ where $z_{2}=z_{1} \bigvee t\left(y_{2}^{\prime}-x_{2}\right)$, so that $\left[z_{2}\right] \leqq(6 \epsilon)[e]$. Now $t x_{2} y_{2}^{\prime}$ is less than or equal to $x_{2}$, and is equivalent to a sub-element of $x_{1}$ (for $t$ is); hence if $h_{1}, h_{2}$ denote maximal equivalent subelements of $x_{1}, x_{2}$, respectively, 7.5 shows that there exists $k_{2} \leqq h_{2}$ such that $t x_{2} y_{2}^{\prime} \sim k_{2}$. Then we have $x_{2}-h_{2} \leqq x_{2}-k_{2} \sim x_{2}-t x_{2} y_{2}^{\prime} \leqq\left(x_{2}-y_{2}^{\prime}\right) \bigvee z_{2}$, so that $\left[x_{2}-h_{2}\right] \leqq(9 \epsilon)[e]$. This holds for every $\epsilon$ sufficiently small, so that $x_{2}=h_{2}$. 
Similarly $x_{1}=h_{1}$, and therefore $x_{1} \sim x_{2}$.

The isomorphism between $(E, \sim)$ and $P \otimes U$ is now established $\left({ }^{21}\right)$.

18. Imbedding of $E\left(e^{\prime}\right)$ in a direct product.

18.1. Having fully considered the relative algebras $E\left(e_{n}\right)$, we turn now to $E\left(e^{\prime}\right)$, where $e^{\prime}$ is the greatest bounded invariant element disjoint from all indecomposable elements $(10.2,10.3)$. It was shown in 13.4 that $e^{\prime}=\mathrm{V} e_{n}$, that the elements $e_{n}$ are disjoint, and that $\left[e_{n}\right]=\rho_{n}\left[\bar{e}_{n}\right]$ for each $n$ (where $\rho_{n}$ is rational and $\left.0<\rho_{n} \leqq 1\right)$. In the preceding section it was shown that each principal ideal $\left(E\left(e_{n}\right), \sim\right)$ is isomorphic to the direct product (say) $P_{n} \otimes U_{n}$, where $P_{n}$ is a (relative) sub-algebra of $E\left(e_{n}\right)$ which is (to within isomorphism) a numerical measure algebra $\left(I^{1}\right.$ or $\left.I^{\mathfrak{m}}\right)$, and $U_{n}$ is the (relative) algebra of relatively invariant elements in $E\left(e_{n}\right)$. Since $U_{n}=e_{n} U$ is isomorphic, in a natural way, to the principal ideal $U\left(\bar{e}_{n}\right)(8.3)$, we have an isomorphism $\phi_{n}$ mapping $\left(E\left(e_{n}\right), \sim\right)$ on $P_{n} \otimes U\left(\bar{e}_{n}\right)$.

To combine these $\phi_{n}$ 's into an isomorphism of $E\left(e^{\prime}\right)$, we first define a numerical measure algebra $J^{\prime}$ as follows: $J^{\prime}$ is the direct sum of the measure algebras $\rho_{n} P_{n}$ obtained by multiplying all measures in $P_{n}$ by $\rho_{n}$. That is, $J^{\prime}$ is the set of all sequences $x=\left(x_{n}\right), x_{n} \in P_{n}$, in which algebraic operations are defined in the obvious way (for example, $\left(x_{n}\right) \bigvee\left(y_{n}\right)=\left(x_{n} \bigvee y_{n}\right)$ ), and in which a (numerical) measure is defined by: $\mu^{\prime}(x)=\sum \rho_{n} \mu_{n}\left(x_{n}\right), \mu_{n}$ being the measure in $P_{n}$. It is easy to see that $J^{\prime}\left(=\left(J^{\prime}, \mu^{\prime}\right)\right)$ is a non-atomic numerical measure algebra in which the measure is at most countably infinite, so that it satisfies our postulates. We use $j^{\prime}$. to denote the unit-element in $J^{\prime}$ (that is, the sequence $\left(e_{n}\right)$ ), and $j_{n}$ to denote the element of $J^{\prime}$ whose " $n$th coordinate" is $e_{n}$ and whose other coordinates are all $o$. The ideal $J^{\prime}\left(j_{n}\right)$ is thus isomorphic to $\left(P_{n}, \mu_{n}\right)$; and to save notation we shall usually not distinguish between them. Note that they are not isometric, in general; we have, for $x \in J^{\prime}\left(j_{n}\right)$, $\mu^{\prime}(x)=\rho_{n} \mu_{n}(x)$. We shall prove that $\left(E\left(e^{\prime}\right), \sim\right)$ is isomorphic to a principal ideal in $J^{\prime} \otimes U\left(e^{\prime}\right)$.

All considerations in the present section will be relative to $e^{\prime}$, so we write $e^{\prime}$ as $e$ and $E\left(e^{\prime}\right)$ as $E$ in what follows-noting for future use that, since $e^{\prime}$ is invariant, the relatively invariant elements form the ideal $U\left(e^{\prime}\right)$.

18.2. Let $R, S$ be the representation spaces of $J^{\prime}$ and $U$ respectively. To the element $j_{n} \in J^{\prime}$ there corresponds an open-closed subset $j_{n}^{*} \subset R$; and it is easy to see that $j_{n}^{*}$ is homeomorphic to the representation space $R_{n}$ of $J^{\prime}\left(j_{n}\right)$ 一that is, of $P_{n}$. Thus we can take $R_{n}=j_{n}{ }^{*}$, so that $U R_{n}$ is residual in $R$. Similarly, we can take the representation space $S_{n}$ of $U\left(\bar{e}_{n}\right)$ to be the openclosed subset $\bar{e}_{n}^{*}$ of $S$ which corresponds to $\bar{e}_{n} \in U$, and have that $U S_{n}$ is residual in $S$. It is easy to see that the direct product $P_{n} \otimes U\left(\bar{e}_{n}\right)$ is isomorphic

(21) Substantially the same arguments prove that the "direct product" $J \otimes U$, as characterized in 3.1 (e), is isomorphic to the product constructed in $\$ 4$. More precisely, the arguments apply provided that $J$ is non-atomic and of finite total measure; and the general case can be reduced to this one. 
to the principal ideal of $\left\{R_{n} \times S_{n}\right\}$ in $J^{\prime} \otimes U$, and we identify them accordingly. We write $M^{\prime}\{H\}$ for the measure-function on $J^{\prime} \otimes U$, and $M_{n}\{H\}$ for the measure-function on $P_{n} \otimes U\left(\bar{e}_{n}\right)$, with the natural convention that $M_{n}$ is always 0 for each $s \in S-S_{n}$. Thus, if $H \subset j_{n}^{*} \times e_{n}^{*}, M^{\prime}\{H\}=\rho_{n} M_{n}\{H\}$.

Note that, while the sets $R_{n}$ are necessarily disjoint (for the elements $j_{n}$ are), the sets $S_{n}$ are not disjoint in general (for the same is true of the elements $\left.\bar{e}_{n}\right)$.

For each $n$ we have an isomorphism $\phi_{n}$ between $E\left(e_{n}\right)$ and the ideal of $\left\{j_{n}^{*} \times e_{n}^{*}\right\}\left(=\left\{R_{n} \times S_{n}\right\}\right)$ in $J^{\prime} \otimes U$. Define, for each $x \in E, \phi(x)=V_{\phi_{n}}\left(x e_{n}\right)$. It is immediate that $\phi$ is an algebraic $\left(\sigma_{-}\right)$isomorphism between $E$ and the principal ideal of $\mathrm{V}\left\{j_{n}^{*} \times e_{n}^{*}\right\}$ in $J^{\prime} \otimes U$. We have only to verify that $\phi$ is "measure-preserving" both ways.

18.3. If $x \leqq e_{n}, x^{\prime} \leqq e_{m}$, and $x \sim x^{\prime}$, then $M^{\prime}\{\phi(x)\}=M^{\prime}\left\{\phi\left(x^{\prime}\right)\right\}$.

If $m=n$ this follows from the corresponding property of $\phi_{n}$; thus we can assume $m \neq n$. Now, as was shown in $\$ 17$, there exists $y \leqq e_{n}$ such that (i) $\left[x+{ }_{2} y\right] \leqq \epsilon\left[e_{n}\right]$, (ii) $M_{n}\left\{\phi_{n}(x)+{ }_{2} \phi_{n}(y)\right\} \leqq \epsilon$, and (iii) $y=\bigvee_{p_{i}} v_{i}$ where $p_{i} \in P_{n}$, $v_{i} \in U_{e_{n}}=e_{n} U$, and the elements $v_{i}$ are disjoint and nonzero. Thus $v_{i}=e_{n} u_{i}$, say, where $u_{i} \in U\left(\bar{e}_{n}\right)$ (cf. 8.2) and the invariant elements $u_{i}$ are disjoint; and, since $p_{i} \leqq e_{n}$, we have $y=\mathrm{V}_{p_{i}} u_{i}$. Similarly there exists $y^{\prime} \leqq e_{m}$ such that ( $\left.\mathrm{i}^{\prime}\right)$ $\left[x^{\prime}+{ }_{2} y^{\prime}\right] \leqq \epsilon\left[e_{m}\right]$, (ii') $M_{m}\left\{\phi_{m}\left(x^{\prime}\right)+{ }_{2} \phi_{m}\left(y^{\prime}\right)\right\} \leqq \epsilon$, and (iii') $y=\bigvee q_{j} u_{j}^{\prime}$, where $q_{j} \in P_{m}, u_{j}^{\prime} \in U\left(\bar{e}_{m}\right)$, and the invariant elements $u_{j}^{\prime}$ are disjoint. Now enumerate the nonzero elements $u_{i} u_{j}^{\prime}, u_{i}-\bar{e}_{m}, u_{j}^{\prime}-\bar{e}_{n}$, into a sequence $w_{k}$; thus the elements $w_{k}$ are disjoint and invariant, and we may write $y=\bigvee r_{k} w_{k}, y^{\prime}=\bigvee s_{k} w_{k}$, where $r_{k} \in P_{n}$ and $s_{k} \in P_{m}$.

We have $\left[w_{k} y\right]=\left[r_{k} e_{n} w_{k}\right]=\mu_{n}\left(r_{k}\right)\left[e_{n} w_{k}\right]$, from the fundamental property of $P_{n}$; but $\left[e_{n}\right]=\rho_{n}\left[\bar{e}_{n}\right]$, and therefore $(12.13)\left[e_{n} w_{k}\right]=\rho_{n}\left[w_{k}\right]$ if $w_{k} \leqq \bar{e}_{n}$. If $w_{k}$ is not $\leqq \bar{e}_{n}$, then $w_{k} \leqq-\bar{e}_{n}$, by construction, so that there is no loss in supposing $r_{k}=o$. Thus (using 12.20) we see that, in all cases, $\left[w_{k} y\right]=\rho_{n} \mu_{n}\left(r_{k}\right)\left[w_{k}\right]$. Similarly $\left[w_{k} y^{\prime}\right]=\rho_{m} \mu_{m}\left(s_{k}\right)\left[w_{k}\right]$. But since $x \sim x^{\prime}$, we have $\left[w_{k} x\right]=\left[w_{k} x^{\prime}\right]$; and by arguments similar to those in 17.10 it follows that $\left|\rho_{n} \mu_{n}\left(r_{k}\right)-\rho_{m} \mu_{m}\left(s_{k}\right)\right|$ $\leqq 2 \epsilon$. Thus $\left|\rho_{n} M_{n}\left\{\phi_{n}(y)\right\}-\rho_{m} M_{m}\left\{\phi_{m}\left(y^{\prime}\right)\right\}\right| \leqq 2 \epsilon$, whence $\mid \rho_{n} M_{n}\left\{\phi_{n}(x)\right\}$ $-\rho_{m} M_{m}\left\{\phi_{m}\left(x^{\prime}\right)\right\} \mid \leqq 4 \epsilon$. That is, $\left|M^{\prime}\{\phi(x)\}-M^{\prime}\left\{\phi\left(x^{\prime}\right)\right\}\right| \leqq 4 \epsilon$ for every $\epsilon$, so that $M^{\prime}\{\phi(x)\}=M^{\prime}\left\{\phi\left(x^{\prime}\right)\right\}$.

18.4. If $x \sim x^{\prime}$, then $M^{\prime}\{\phi(x)\}=M^{\prime}\left\{\phi\left(x^{\prime}\right)\right\}$.

From 6.2, we can write $x^{\prime}=V_{x_{m}}{ }^{\prime}$ where the elements $x_{m}^{\prime}$ are disjoint, and $x_{m}^{\prime} \sim x e_{m}$. Similarly we can write $x e_{m}=\mathrm{V}_{n} x_{m n}$ where the elements $x_{m n}$ are disjoint and $x_{m n} \sim x_{m}{ }^{\prime} e_{n}$. From 18.3 we have $M^{\prime}\left\{\phi\left(x_{m n}\right)\right\}=M^{\prime}\left\{\phi\left(x_{m}{ }^{\prime} e_{n}\right)\right\}$; and the countable additivity of $M^{\prime}$ then gives $M^{\prime}\{\phi(x)\}=M^{\prime}\left\{\phi\left(x^{\prime}\right)\right\}$.

18.5. If $M^{\prime}\{\phi(x)\}=M^{\prime}\left\{\phi\left(x^{\prime}\right)\right\}$ then $x \sim x^{\prime}$.

By an argument similar to that in 18.4 , we see that it will be enough to prove this assuming $x \leqq e_{m}$ and $x^{\prime} \leqq e_{n}$. Let $y, y^{\prime}$ be maximal equivalent subelements of $x, x^{\prime}$, respectively (7.5), and write $z=x-y, z^{\prime}=x^{\prime}-y^{\prime}$. By 18.4 , $M^{\prime}\{\phi(y)\}=M^{\prime}\left\{\phi\left(y^{\prime}\right)\right\}$; also here $M^{\prime}\{\phi(x)\}<\infty$; hence the additivity of $M^{\prime}$ 
gives $M^{\prime}\{\phi(z)\}=M^{\prime}\left\{\phi\left(z^{\prime}\right)\right\}$. Now it is clear from the construction in $\$ 17$ that $M^{\prime}\{\phi(z)\}$ has the value 0 for all $s \in S-\bar{z}^{*}$; similarly $M^{\prime}\left\{\phi\left(z^{\prime}\right)\right\}=0$ outside $\bar{z}^{\prime *}$. Since $\bar{z} \bar{z}^{\prime}=o$, we have $\bar{z}^{*} \cap \bar{z}^{\prime *}=0$, and therefore $M^{\prime}\{\phi(z)\}$ $=M^{\prime}\left\{\phi\left(z^{\prime}\right)\right\}=0$ for all $s \in S$. Since $M_{m}\left\{\phi_{m}(z)\right\}=0$, we have $z=0$; similarly $z^{\prime}=0$, so that $x=y \sim y^{\prime}=x^{\prime}$.

The proof is now complete; but for later use we note the property:

18.6. If $u \in U, M^{\prime}\{\phi(u)\}$ is the characteristic function of $u^{*}$.

We have $\left[e_{n} u\right]=\rho_{n}\left[\bar{e}_{n} u\right]$, and so, since $\phi$ is an isomorphism, $M^{\prime}\left\{\phi\left(e_{n} u\right)\right\}$ $=\rho_{n} M^{\prime}\left\{\phi\left(\bar{e}_{n} u\right)\right\}$. But $M^{\prime}\left\{\phi\left(e_{n} u\right)\right\}=\rho_{n} M_{n}\left\{\phi_{n}\left(e_{n} u\right)\right\}$, by definition; hence $M^{\prime}\left\{\phi\left(\bar{e}_{n} u\right)\right\}=M_{n}\left\{\phi_{n}\left(e_{n} u\right)\right\}$. Since $e_{n} u$ is invariant relative to $e_{n}$, the construction in $\$ 17$ shows that $M_{n}\left\{\phi_{n}\left(e_{n} u\right)\right\}$ is the characteristic function of the open-closed subset of $S_{n}$ which "corresponds" to $e_{n} u$; and since the (absolute) invariant element corresponding to $e_{n} u$ in the isomorphism of 8.3 is $\bar{e}_{n} u$, we here have $M^{\prime}\left\{\phi\left(\bar{e}_{n} u\right)\right\}=$ characteristic function of $\left(\bar{e}_{n} u\right)^{*}$.

It readily follows that $M^{\prime}\left\{\phi\left(u\left(\bar{e}_{n}-\bar{e}_{1}-\bar{e}_{2}-\cdots-\bar{e}_{n-1}\right)\right)\right\}=$ characteristic function of $u^{*} \cap\left(\bar{e}_{n}^{*}-\bar{e}_{1}^{*}-\bar{e}_{2}^{*}-\cdots-\bar{e}_{n-1}^{*}\right)$; and on summing (and neglecting a subset of $S$ of the first category) we obtain $M^{\prime}\{\phi(u)\}=$ characteristic function of $u^{*}$.

19. The representation of $(E, \sim)$.

19.1 We begin by imbedding $E\left(e^{\prime \prime}\right)$ in a direct product, where $e^{\prime \prime}$ is the element defined in 10.2. It has been shown (10.6) that $e^{\prime \prime}=V f^{n}$, where the elements $f^{n}$ are disjoint, bounded and equivalent, and where $\bar{f}^{n}=e^{\prime \prime}$, for each $n$. Now $E\left(f^{n}\right)$ has, relative to $f^{n}$, all the properties which were used in studying $E\left(e^{\prime}\right)$. Hence the imbedding obtained in $\$ 18$ for $E\left(e^{\prime}\right)$ applies also to each $E\left(f^{n}\right)$. Remembering that the algebra $U_{f^{n}}$ of invariant elements relative to $f^{n}$ is isomorphic to $U\left(\bar{f}^{n}\right)(8.3)$ - that is, to $U\left(e^{\prime \prime}\right)$ - we thus have, for each $n$, an isomorphism $\psi_{n}$ between $\left(E\left(f^{n}\right), \sim\right)$ and a principal ideal in a direct product (say) $J^{n} \otimes U\left(e^{\prime \prime}\right), J^{n}$ being a non-atomic numerical measure algebra. Let $J^{\prime \prime}$ denote the "direct sum" of the measure algebras $J^{n}$-that is, $J^{\prime \prime}$ is the set of sequences $x=\left(x^{n}\right), x^{n} \in J^{n}$, with algebraic operations defined "co-ordinatewise," and with a (numerical) measure $\mu^{\prime \prime}$ defined by: $\mu^{\prime \prime}(x)=\sum \mu^{n}\left(x^{n}\right), \mu^{n}$ being the measure in $J^{n}$. We now show that $E\left(e^{\prime \prime}\right)$ is isomorphic to a principal ideal in $J^{\prime \prime} \otimes U\left(e^{\prime \prime}\right)$.

As in 18.1 and 18.2, we can express the unit-element $j^{\prime \prime}$ of $J^{\prime \prime}$ as $V j^{n}$, where the elements $j^{n}$ are disjoint and the principal ideal $J^{\prime \prime}\left(j^{n}\right)$ can be regarded as being $J^{n}$. Also as before, the open-closed subset $j^{n *}$ of the representation space $R$ of $J^{\prime \prime}$ can be regarded as the representation space $R^{n}$ of $J^{n}$; and the principal ideal of $\left\{R^{n} \times S\right\}$ in $J^{\prime \prime} \otimes U\left(e^{\prime \prime}\right)$, where $S$ is the representation space of $U\left(e^{\prime \prime}\right)$, can be regarded as being $J^{n} \otimes U\left(e^{\prime \prime}\right)$. We use $M^{n}$ for the measure-function in $J^{n} \otimes U\left(e^{\prime \prime}\right)$, and $M^{\prime \prime}$ for the measure-function in $J^{\prime \prime} \otimes U\left(e^{\prime \prime}\right) ;$ thus $M^{\prime \prime}\{H\}=\sum M^{n}\left\{H \cap\left(j^{n *} \times S\right)\right\}$.

We define, for each $x \leqq e^{\prime \prime}, \psi(x)=\bigvee \psi_{n}\left(x f^{n}\right)$; trivially $\psi$ is an algebraic $(\sigma-)$ isomorphism between $E\left(e^{\prime \prime}\right)$ and the principal ideal of $\bigvee \psi_{n}\left(f^{n}\right)$ in 
$J^{\prime \prime} \otimes U\left(e^{\prime \prime}\right)$. All that remains is to verify that $\psi$ is "measure-preserving"; and as in doing this all considerations will be relative to $e^{\prime \prime}$, we may suppose $e^{\prime \prime}=e$ in 19.2 and 19.3 .

19.2. If $x \sim y, M^{\prime \prime}\{\psi(x)\}=M^{\prime \prime}\{\psi(y)\}$.

By the same argument as in 18.4 , it is enough to prove this when $x \leqq f^{m}$ and $y \leqq f^{n}$. Now from 13.1 applied relative to $f^{m}$ (so that the "invariant elements" are of the form $\left.u f^{m}, u \in U\right)$, there exist, for any given integer $N$, elements $u_{i} \in U(1 \leqq i \leqq N)$ such that $f^{m} u_{i} u_{j}=o$ when $i \neq j, \vee u_{i} \geqq f^{m}$ (so that $\left.V u_{i}=\bar{f}^{m}=e\right)$, and $((i-1) / N)\left[u_{i} f^{m}\right] \leqq\left[u_{i} x\right] \leqq(i / N)\left[u_{i} f^{m}\right]$. From 5.10 it follows that the elements $u_{i}$ are actually disjoint. Choose $t_{i} \leqq u_{i} x$ with $\left[t_{i}\right]$ $=((i-1) / N)\left[u_{i} f^{m}\right]$, and write $z_{i}=u_{i} x-t_{i}$ and $z=\bigvee_{z_{i}}$. Thus $x=\bigvee t_{i} \bigvee z$, where the elements $t_{i}$ and $z$ are disjoint, and (from 12.5(5)) $[z] \leqq(1 / N)\left[f^{m}\right]$.

Since $y \sim x$, we can (6.2) write $y=\bigvee t_{i}^{\prime} \vee z^{\prime}$, where the elements $t_{i}^{\prime}$ and $z^{\prime}$ are disjoint, $t_{i}^{\prime} \sim t_{i}$, and $z^{\prime} \sim z$.

Now $u_{i} f^{m}$ is invariant relative to $f^{m}$; hence (18.6) $M^{m}\left\{\psi_{m}\left(u_{i} f^{m}\right)\right\}$ is the characteristic function of the open-closed subset of $S$ which "corresponds" to $u_{i} f^{m}$. Here $u_{i} f^{m}$ was replaced by $u_{i} \bar{f}^{m}=u_{i}$ in the isomorphism between $U_{f^{m}}$ and $U\left(=U\left(e^{\prime \prime}\right)\right)$; thus we have $M^{m}\left\{\psi_{m}\left(u_{i} f^{m}\right)\right\}=1$ for $s \in u_{i}^{*}, 0$ for $s \in S-u_{i}^{*}$. Since $\psi_{m}$ is an isomorphism, the relation $\left[t_{i}\right]=((i-1) / N)\left[u_{i} f^{m}\right]$ gives $M^{\prime \prime}\left\{\psi\left(t_{i}\right)\right\}=(i-1) / N$ for $s \in u_{i}^{*}, 0$ for $s \in S-u_{i}^{*}$. Similarly we have $M^{\prime \prime}\{\psi(z)\}$ $\leqq 1 / N$ for all $s$.

Again, we have $f^{m} \sim f^{n}$ and therefore (6.6) $u_{i} f^{m} \sim u_{i} f^{n}$. Thus $\left[t_{i}^{\prime}\right]$ $=((i-1) / N)\left[u_{i} f^{n}\right]$, so by the same reasoning as before (applied to the isomorphism $\left.\psi_{n}\right)$ we have $M^{\prime \prime}\left\{\psi\left(t_{i}^{\prime}\right)\right\}=(i-1) / N$ for $s \in u_{i}^{*}, 0$ for $s \in S-u_{i}^{*}$, and $M^{\prime \prime}\left\{\psi\left(z^{\prime}\right)\right\} \leqq 1 / N$.

Thus from the additivity of $M^{\prime \prime}$, we have $\mid M^{\prime \prime}\{\psi(x)\}-M^{\prime \prime}\{\psi(y)\}$ $\leqq 1 / N$; and since $N$ here was arbitrary, it follows that $M^{\prime \prime}\{\psi(x)\}$ $=M^{\prime \prime}\{\psi(y)\}$.

19.3. If $M^{\prime \prime}\{\psi(x)\}=M^{\prime \prime}\{\psi(y)\}$, then $x \sim y$.

This follows by the same argument as in 18.5. The desired isomorphic imbedding of $\left(E\left(e^{\prime \prime}\right), \sim\right)$ is thus established.

19.4. It is now easy to extend the imbedding to one of all of $E$. For convenience of exposition, we assume that none of the elements $e^{o}, e^{\prime}, e^{\prime \prime}$ (cf. 9.6, 10.2) is zero; the modifications needed otherwise are obvious. We have from 9.7 an isomorphism $\theta$ of $E\left(e^{o}\right)$ on a principal ideal in $K \otimes U\left(e^{o}\right)$, and from $\$ 18$ an isomorphism $\phi$ of $E\left(e^{\prime}\right)$ on a principal ideal in $J^{\prime} \otimes U\left(e^{\prime}\right)$. We have just constructed an isomorphism $\psi$ of $E\left(e^{\prime \prime}\right)$ on a principal ideal in $J^{\prime \prime} \otimes U\left(e^{\prime \prime}\right)$. Further, the elements $e^{o}, e^{\prime}, e^{\prime \prime}$ are disjoint and invariant, and $e^{o} \bigvee e^{\prime} \bigvee e^{\prime \prime}=e$.

Now form the usual direct sum $J=J^{\prime} \oplus J^{\prime \prime}$ of the numerical measure algebras $J^{\prime}, J^{\prime \prime}$; thus $J$ consists of the pairs $\left(x^{\prime}, x^{\prime \prime}\right)\left(x^{\prime} \in J^{\prime}, x^{\prime \prime} \in J^{\prime \prime}\right)$, with measure $\mu$ given by $\mu\left(x^{\prime}, x^{\prime \prime}\right)=\mu^{\prime}\left(x^{\prime}\right)+\mu^{\prime \prime}\left(x^{\prime \prime}\right), \mu^{\prime}$ and $\mu^{\prime \prime}$ being the measures in $J^{\prime}$ and $J^{\prime \prime}$. As a non-atomic algebra of at most countably infinite measure, $J$ satisfies our postulates. Let $\mathcal{E}$ denote the free direct sum (cf. $3.1(\mathrm{~d})$ ) of the 
direct products $K \otimes U\left(e^{o}\right)$ and $J \otimes U\left(-e^{o}\right)$. To $x \in E$ we make correspond the pair $\left(\theta\left(x e^{o}\right), \phi\left(x e^{\prime}\right) \vee \psi\left(x e^{\prime \prime}\right)\right)$ in $\varepsilon$. (The products $J^{\prime} \otimes U\left(e^{\prime}\right)$ and $J^{\prime \prime} \otimes U\left(e^{\prime \prime}\right)$ are regarded as imbedded in $J \otimes U\left(e^{\prime} \vee e^{\prime \prime}\right)$ in the obvious way.) This evidently produces an algebraic isomorphism between $E$ and the principal ideal of the element $\left(\theta\left(e^{o}\right), \phi\left(e^{\prime}\right) \bigvee \psi\left(e^{\prime \prime}\right)\right)$ in $\varepsilon$. Further, if $x \sim y$, then (since $e^{o}, e^{\prime}$ and $e^{\prime \prime}$ are invariant) 6.6 gives $x e^{\circ} \sim y e^{\circ}, x e^{\prime} \sim y e^{\prime}$ and $x e^{\prime \prime} \sim y e^{\prime \prime}$, so that the corresponding elements of $\mathcal{E}$ are equivalent in $\mathcal{E}$. Conversely, if $x, y \in E$ correspond to equivalent elements in $\varepsilon$, we have $x e^{o} \sim y e^{o}$ and also $M\left\{\phi\left(x e^{\prime}\right)\right\}$ $+M\left\{\psi\left(x e^{\prime \prime}\right)\right\}=M\left\{\phi\left(y e^{\prime}\right)\right\}+M\left\{\psi\left(y e^{\prime \prime}\right)\right\}, M$ denoting the measure function in $J \otimes U\left(-e^{o}\right)$. But $M\left\{\phi\left(x e^{\prime}\right)\right\}$ is a function on the representation space $S$ of $U\left(-e^{o}\right)$ which vanishes outside the open-closed subset $e^{*}$ corresponding to $e^{\prime}$. Similarly $M\left\{\psi\left(x e^{\prime \prime}\right)\right\}$ vanishes outside $e^{\prime \prime *}$. Since $e^{\prime *} \cap e^{\prime *}=0$, and $e^{\prime *} \cup e^{\prime \prime *}=S$, we obtain $M^{\prime}\left\{\phi\left(x e^{\prime}\right)\right\}=M^{\prime}\left\{\phi\left(y e^{\prime}\right)\right\}$ and $M^{\prime \prime}\left\{\psi\left(x e^{\prime \prime}\right)\right\}$ $=M^{\prime \prime}\left\{\psi\left(y e^{\prime \prime}\right)\right\}$, so that $x e^{\prime} \sim y e^{\prime}$ and $x e^{\prime \prime} \sim y e^{\prime \prime}$. Hence, finally, $x \sim y$, and the main theorem of this paper is proved: $(E, \sim)$ is isomorphic to a principal ideal in the free direct sum of $K \otimes U\left(e^{o}\right)$ and $J \otimes U\left(-e^{o}\right)$, each of the two summands being the direct product of a numerical measure algebra with a trivial abstract measure algebra.

19.5. The above argument would evidently also prove that $(E, \sim)$ is isomorphic to a principal ideal in the single direct product $(K \oplus J) \otimes U$. This way of stating the theorem has the drawback that the numerical measure algebra $K \oplus J$ will in general not satisfy postulate II (for $K$ has atoms of measure 1 , and $J$ has in general non-atomic elements of the same measure), so that $(K \oplus J) \otimes U$ will in general not satisfy our postulates. However, it is immediate from this alternative formulation of the theorem that $(E, \sim)$ is naturally isomorphic to an abstract measure alegbra $(E, M)$ in which the values of the abstract measure $M$ are equivalence classes of numerical functions (more precisely, non-negative real-valued continuous functions on the representation space of $U$, modulo functions which vanish outside a set of first category), $M$ being countably additive in the obvious sense. Incidentally this proves that any equivalence relation satisfying our postulates (2.1) is induced by a "reasonable" countably additive abstract measure $\lambda$.

20. Corollaries and applications.

20.1. In this section we mention briefly some deductions from the preceding general theory. There is, of course, an application to the theory of abstract-valued integration, which will be treated in a subsequent paper. Of the deductions to be considered here, the decomposition theorem given in $20.4(2)$ below is probably the most significant.

20.2. Some special cases.

(1) Consider the elements $a_{n}$ given by 11.7 ; thus they are disjoint, $\mathrm{V} a_{n}=e^{\prime}$ (10.2), and $a_{n}$ is homogeneous of infinite order $\mathfrak{m}_{n}$ over $U$. We observe that the arguments of $\$ \$ 14-17$ apply to $a_{n}$ as well as to $e_{n}$. Thus each principal ideal $\left(E\left(a_{n}\right), \sim\right)$ is isomorphic to the direct product $I^{\mathfrak{m}_{n}} \otimes U\left(a_{n}\right)$. 
(2) In particular, if all nonzero elements of $E$ are decomposable and bounded (that is, if $e=e^{\prime}$ ), and if $E$ is separable over $U$ (that is, if $e$ is of order $\leqq \aleph_{0}$, and thus of order exactly $\boldsymbol{\aleph}_{0}$, over $\left.U\right),(E, \sim)$ is isomorphic to $I^{1} \otimes U-$ giving a characterization of the direct product of $I^{1}$ with a "trivial" algebra.

20.3. The numerical case.

(1) Characterizations. It is easy to derive characterizations of numerical measure algebras (satisfying our postulates-notably II; cf. 2.2). From the remark in footnote 6 , it is enough to restrict attention to the case in which $E$ is non-atomic. We then have the theorem:

If $E$ is a non-atomic algebra, a necessary and sufficient condition that $(E, \sim)$ be isomorphic to a numerical measure algebra is that $(E, \sim)$ satisfies the postulates of 2.1 and has $o$ and $e$ for its only invariant elements.

An immediate consequence of this is (cf. [10, p. 423]):

If $E$ is non-atomic, $(E, \sim)$ (satisfying the postulates of 2.1 ) is isomorphic to a numerical measure algebra if and only if, for every $x, y \in E$, either $[x] \leqq[y]$ or $[y] \leqq[x]$.

(2) Decomposition into homogeneous parts. From 20.2(1) we have at once the theorem (cf. [11]):

$A$ non-atomic numerical measure algebra $(E, \mu)$ (with $\sigma$-finite measure, of course) is the direct sum of a countable number (at most) of homogeneous measure algebras $\left(E\left(a_{n}\right), \mu\right)$; and $\left(E\left(a_{n}\right), \mu\right)$ is isomorphic to $I^{\mathfrak{m}_{n}}$ (and thus isometric with $I^{\mathfrak{m}_{n}}$, to within a multiplicative nonzero constant).

20.4. Groups of measure-preserving automorphisms.

(1) Equivalence with respect to $\Phi$. Let $E$ be a given algebra (satisfying the countable chain condition), and let a group $\Phi$ of automorphisms $\phi$ of $E$ be given. Write $x \sim_{\Phi} y$ to mean that there exist sequences $\left\{x_{n}\right\},\left\{y_{n}\right\}$, each disjoint, in $E$, and automorphisms $\phi_{n} \in \Phi$, such that $x=\vee x_{n}, y=V y_{n}$, and $y_{n}=\phi_{n}\left(x_{n}\right)$.

One readily sees that $\sim_{\Phi}$ is an equivalence relation, and that $\left(E, \sim_{\Phi}\right)$ satisfies all the postulates (2.1) except, in general, III. Postulate III will also be satisfied in at least one important case (cf. 20.4(2)).

It is easy to see that $u \in E$ is invariant in $\left(E, \sim_{\Phi}\right)$ if and only if $\phi(u) \leqq u$ for every $\phi \in \Phi$ - that is, $u$ is "invariant under $\Phi$ " in the usual sense.

(2) Decomposition with respect to $\Phi$.

TheOREM. Let $(E, m)$ be a numerical measure algebra in which $m$ is an arbitrary reduced $\sigma$-finite numerical measure (cf. 2.2 ; postulate II need not be satisfied); and let $\Phi$ be a given group of measure-preserving automorphisms of $E$. Then there exist disjoint nonzero elements $c_{n} \in E$, each invariant under $\Phi$, and sub-algebras $A_{n}, B_{n}$ of $E\left(c_{n}\right)$ and reduced $\sigma$-finite numerical measures $\mu_{n}$ on $A_{n}, \nu_{n}$ on $B_{n}$, such that:

(i) $V c_{n}=e$.

(ii) $B_{n}$ consists of exactly those elements of $E\left(c_{n}\right)$ which are invariant under $\Phi$.

(iii) If $x, y \in A_{n}, \mu_{n}(x)=\mu_{n}(y)$ if and only if $x \sim_{\Phi} y$ (in $E$ ). 
(iv) $\left(A_{n}, \mu_{n}\right)$ is isometric to one of the following:

(a) $I^{\mathfrak{m}_{n}}$ (for some cardinal $\mathfrak{m}_{n} ; c f .3 .1(\mathrm{a})$ ),

(b) the direct sum of $\aleph_{0}$ copies of $I^{\mathfrak{m}_{n}}$,

(c) the algebra generated by a finite number of atoms each of measure 1,

(d) $K(c f .3 .1(\mathrm{a}))$,

and no two of the measure algebras $\left(A_{n}, \mu_{n}\right)$ are isomorphic.

(v) $\left(E\left(c_{n}\right), m\right)$ is the "direct product" of $\left(A_{n}, \mu_{n}\right)$ and $\left(B_{n}, \nu_{n}\right)$; that is, $E\left(c_{n}\right)=c_{n} B\left(A_{n} \cup B_{n}\right)$ and, whenever $x \in A_{n}$ and $z \in B_{n}, m(x z)=\mu_{n}(x) \nu_{n}(z)$ (with the convention $0 \cdot \infty=0=\infty \cdot 0)$.

REMARKS. Before sketching the proof, we note the following consequences:

(a) For any $x, y \in A_{n}$ we have $\left[\mu_{n}(x)=\mu_{n}(y)\right] \leftrightarrow\left[x \sim_{\Phi} y\right] \rightarrow[m(x)=m(y)]$. Hence, if for some $n$ the measure $m$ is $\sigma$-finite on $A_{n}$ (that is, $c_{n}=\mathrm{V} x_{k}$ where $m\left(x_{k}\right)<\infty$ and $\left.x_{k} \in A_{n}\right)$, it follows that $\mu_{n}$ is then a nonzero finite constant multiple of $m$. If further $m\left(c_{n}\right)<\infty$, the product formula shows that $\mu_{n}$ and $\nu_{n}$ are both nonzero finite constant multiples of $m$. In particular, this is the case if $m(e)<\infty$.

(b) On applying this theorem to the algebra of measurable sets modulo null sets of a separable measure space, one can derive sharpened forms of theorems of von Neumann [13 p. 617] and Halmos [7, Theorem 2] $\left.{ }^{22}\right)$, the direct sum decompositions there given being replaced (roughly speaking) by representations as direct products of measure spaces. It is hoped that details may appear elsewhere.

Proof of theorem. In the present case, $\left(E, \sim_{\Phi}\right)$ can easily be seen to satisfy postulate III, so the preceding theory applies to it. We suppose for simplicity that $e=e^{\prime}$ (cf. 10.2). Then (11.7, with a slight change in notation) $e=\mathrm{V} c_{n}$, where the elements $c_{n}$ are disjoint, nonzero, and homogeneous over $U$. It is easily seen that each $c_{n}$ is now invariant. Further, in view of 20.2(1), $\left(E\left(c_{n}\right), \sim_{\Phi}\right)$ is isomorphic to the direct product $A_{n} \otimes B_{n}$, where $B_{n}=U\left(c_{n}\right)$ and $A_{n}=\left(A_{n}, \mu_{n}\right)$ is a sub-algebra of $\left(E\left(c_{n}\right), \sim_{\Phi}\right)$ which is also a numerical measure algebra (isometric to $I^{\mathrm{m}_{n}}$ ). We define the measure $\nu_{n}$ on $B_{n}$, in the present case, to coincide with $m$, which can easily be seen to be $\sigma$-finite on $B_{n}$. Thus we need only verify that $m(x z)=\mu_{n}(x) m(z)$ for $x \in A_{n}, z \in B_{n}$, and it will suffice to establish this in the special case $\mu_{n}(x)=1 / k, k$ a positive integer. But then there exist $k$ disjoint elements $x_{1}, x_{2}, \cdots, x_{k}$ in $A_{n}$ such that $x_{1}=x, V_{x_{i}}=c_{n}$, and $\mu_{n}\left(x_{i}\right)=1 / k$. Hence $x_{i} \sim_{\Phi} x$, so that ( $z$ being invariant) $x_{i} z \sim_{\Phi} x z$, and therefore $m\left(x_{i} z\right)=m(x z)$. Thus $m(x z)=m(x) / k$, completing the proof.

The ideal $E\left(e^{\prime \prime}\right)(10.2)$ is disposed of similarly, using 10.6; here the measure algebras $\left(A_{n}, \mu_{n}\right)$ will be of the type described in (iv b) of the theorem. Finally $E\left(e^{o}\right)$ (9.6) provides the measure algebras of types (iv c) and (iv d).

(3) The Banach-Tarski theorem. Apply the preceding theorem taking

(22) In view of the fact that in [7] and [13] we have the separable case, in which the algebras $A_{n}, B_{n}$ can always be realised as sub-fields of the given field of measurable sets. 
$(E, m)$ to be the algebra of (Lebesgue) measurable sets modulo null sets in Euclidean space, and $\Phi$ to be the group of all rigid motions (or even of all translations). The only invariant elements are $o$ and $e$, and the algebra $E$ is separable. Hence we here have $c_{1}=e$ and $E=A_{1}$. Property (iii) of the theorem (see remark (a)) then gives: $m(x)=m(y)$ if and only if $x \sim_{\Phi} y(m$ denoting Lebesgue measure) - a well known theorem of Banach and Tarski [1, p. 277].

One can go on to obtain such generalizations as the following: For any measurable subset $X$ of the plane, and any real number $a$, let $m_{X}(a)$ denote the (linear) measure of the intersection of $X$ with the line $x=a$. Then if $m_{X}(x)=m_{Y}(x)$ for almost all $x$, we can write $X=Z \cup \cup X_{n}, Y=T \cup \cup Y_{n}$, where $Z$ and $T$ are of (plane) measure zero, each of the sequences $\left\{X_{n}\right\}$, $\left\{Y_{n}\right\}$ is disjoint, the sets $X_{n}$ and $Y_{n}$ are all measurable, and where for each $n$ the sets $X_{n}, Y_{n}$ correspond under some translation parallel to the $y$-axis. (For let $E$ be the algebra of plane measurable sets modulo null sets, and let $\Phi$ be the group of translations parallel to the $y$-axis; it is not hard to see that the abstract measure function $M\{X\}$, in the representation of $\left(E, \sim_{\Phi}\right)$ given by the theorem of 19.5 , is in effect simply the function $m_{X}(x)$.)

\section{BIBLIOGRAPHY}

1. S. Banach and A. Tarski, Sur les décompositions dénombrables, Fund. Math. vol. 6 (1924) pp. 244-277.

2. G. Birkhoff, Lattice theory, Amer. Math. Soc. Colloquium Publications, vol. 25, 1940.

3. - Moore-Smith convergence in general topology, Ann. of Math. vol. 38 (1937) pp. 39-56.

4. G. Choquet, Préliminaires à une nouvelle définition de la mesure, C. R. Acad. Sci. Paris vol. 215 (1942) pp. 52-54.

5. —-, Choix d'une mesure cartésienne $\Delta$; applications, C. R. Acad. Sci. Paris vol. 215 (1942) pp. 101-103.

6. H. Freudenthal, Teilweise geordnete Moduln, K. Akademie van Wetenschappen, Afdeeling Natuurkunde, Proceedings of the Section of Sciences vol. 39 (1936) pp. 647-657.

7. P. Halmos, The decomposition of measures, Duke Math. J. vol. 8 (1941) pp. 386-392.

8. E. R. van Kampen, Infinite product measures and convolutions, Amer. J. Math. vol. 62 (1940) pp. 417-448.

9. L. H. Loomis, On the representation of $\sigma$-complete Boolean algebras, Bull. Amer. Math. Soc. vol. 53 (1947) pp. 757-760.

10. D. Maharam, On measure in abstract sets, Trans. Amer. Math. Soc. vol. 51 (1942) pp. $413-433$

11. - - On homogeneous measure algebras, Proc. Nat. Acad. Sci. U.S.A. vol. 28 (1942) pp. $108-111$.

12. F. J. Murray and J. von Neumann, On rings of operators, Ann. of Math. vol. 37 (1936) pp. 116-229.

13. J. von Neumann, Zur Operatorenmethode in der klassischen Mechanik, Ann. of Math. vol. 33 (1932) pp. 587-642.

14. G. B. Price, The theory of integration, Trans. Amer. Math. Soc. vol. 47 (1940) pp. 1-50.

15. M. H. Stone, The theory of representation for Boolean algebras, Trans. Amer. Math. Soc. vol. 40 (1936) pp. 37-111.

Cambridge, England 